\title{
THE SHAPES IN A CONCRETE CATEGORY
}

\author{
NIKICA UGLEŠIĆ \\ University of Zadar, Croatia
}

\begin{abstract}
We show under what conditions, and how, one can obtain a shape theory (various shape theories) in a concrete category. The technique is, roughly speaking, reduced to the quotients by congruences providing the objects of lower cardinalities. The application yields the new (coarser) classifications in every concrete category which admits sufficiently many non-trivial quotients. Thus, the ordering, (ultra)pseudometric, uniform and topological structures, as well as many algebraic and mixed (multi-) structures, give rise to interesting results.
\end{abstract}

\section{INTRODUCTION}

Although founded purely categorically (after Borsuk's "geometric" approach for compact subsets of the Hilbert cube $([1,2,3])$, a shape theory is, mostly, well known only as the shape theory of topological spaces with respect to spaces having homotopy types of polyhedra $([7,8,13])$. The generalizations founded in [11] and [18] are, primarily, also on that line. (An exception to that kind could be [4], where the shape is treated in somewhat more general categorical frame.) Briefly, the well-studied shape theories are appropriate operable extensions of the homotopy theory to all (i.e., locally bad) spaces. They share many useful invariants with the standard homotopy and bring some new ones ([13, Chapter II], [18, Section 9]).

The key notion in a shape theory is that of expansion ([13, I. 2]). The idea is, as in many other theories, to approximate an object with the "nice" ones. In some cases the inverse limit approach is satisfactory. However, an inverse

2010 Mathematics Subject Classification. 03E99, 06A99, 16D99, 16S99, 20B07, 46A99, 54B15, 54B30, 54C56, 54E99, 55P55.

Key words and phrases. (pointed) set, partially ordered set, (ultra)pseudometric space, topological space, monoid, group, ring, module, vector space, equivalence relation, congruence, (infinite) cardinal, concrete category, quotient object, dimension, expansion, procategory, shape category. 
limit ([9, VIII. 2, 3], [13, I. 2]) is only accidentally an expansion. Namely, although both are pro-morphisms ([13, I. 1]) from an object to an associated inverse system, the corresponding universal properties are quite different:

In order to establish the shape theory of topological spaces, the main problem was to prove the existence of polyhedral ( $A N R-$ ) expansions of noncompact spaces. It was solved by proving the existence of the associated inverse systems called resolutions (and by constructing them), which then are transferred into the homotopy category.

In our approach, for any concrete category $\mathcal{C}$, we firstly propose a subclass of "nice" objects depending on a given cardinal. More precisely, given an infinite cardinal $\kappa$, we choose the full subcategory $\mathcal{C}_{\kappa^{-}}\left(\mathcal{C}_{\kappa}\right)$ of $\mathcal{C}$ determined by all the objects having cardinalities less than (less or equal to) $\kappa$. Then we used to say that the objects of $\mathcal{C}_{\kappa^{-}}\left(\mathcal{C}_{\kappa}\right)$ are nice comparing to those of $\mathcal{C} \backslash \mathcal{C}_{\kappa^{-}}$ $\left(\mathcal{C} \backslash \mathcal{C}_{\kappa}\right)$. Further, we show that every object $X$ admits the canonical $\mathcal{C}_{\kappa^{--}}$ expansion $\left(\mathcal{C}_{\kappa}\right.$-expansion) via the appropriate quotient objects and quotient morphisms by congruences on $X$, denoted by (the notations for objects and morphisms of $\mathcal{C}$ or pro- $\mathcal{C}$ are those of [13]):

$$
\begin{gathered}
\boldsymbol{p}_{\kappa^{-}}=\left(p_{\lambda}\right): X \rightarrow \boldsymbol{X}_{\kappa^{-}}=\left(X_{\lambda}, p_{\lambda \lambda^{\prime}}, \Lambda_{\kappa^{-}}\right) \\
\left(\boldsymbol{p}_{\kappa}=\left(p_{\lambda}\right): X \rightarrow \boldsymbol{X}_{\kappa}=\left(X_{\lambda}, p_{\lambda \lambda^{\prime}}, \Lambda_{\kappa}\right)\right) .
\end{gathered}
$$

Clearly, in the case of $\kappa>|X|(\kappa \geq|X|)$, the inverse system $\boldsymbol{X}_{\kappa^{-}}\left(\boldsymbol{X}_{\kappa}\right)$ has the maximal term, and thus, it is " $\kappa^{-}$-stable" ( $\kappa$-stable"), i.e., isomorphic in pro$\mathcal{C}_{\kappa^{-}}\left(\right.$pro- $\left.\mathcal{C}_{\kappa}\right)$ to the rudimentary (constant) system $\lfloor X\rfloor$. If one wants more special expansions, it has to choose a special subcategory $\mathcal{D} \subseteq \mathcal{C}$ and make the expansions in $\mathcal{D}_{\kappa^{-}}$and $\mathcal{D}_{\kappa}$ (if they exist!). After this, the construction of the corresponding shape categories $\operatorname{Sh}\left(\mathcal{C}, \mathcal{C}_{\kappa^{-}}\right) \equiv S h_{\kappa^{-}}(\mathcal{C}), S h\left(\mathcal{C}, \mathcal{C}_{\kappa}\right) \equiv S h_{\kappa}(\mathcal{C})$ and functors $S_{\kappa^{-}}, S_{\kappa}$ as well as of the relating functors $S_{\kappa^{-}}, S_{\kappa \kappa^{\prime}}, \kappa<\kappa^{\prime}$, follows the general procedure. In the case of $\kappa^{-}$for $\kappa=\aleph_{0}$, we speak about finite shape and denote it by $S h_{0}$.

Figuratively speaking, the quotient shapes of an object are (changeable) "pictures of that object viewing it from outside at different distances". Really, if one wants to estimate ("roughly") a large object in whole and get its global picture, he has to stay outside or to move far enough from the object. Of course, in that case one cannot see a fine (local) structure, and ignores it purposely. Since this kind of shape obviously depends on a given "distance", one, firstly, has to define and fix a distance ("view point for observing"), and, secondly, to propose the "referent" ("nice") objects for the chosen distance, in order to "recognize" ("compare") the shape of a considered object with respect to nice objects at that distance. In our approach, the distance is defined by means of cardinality such that a larger (infinite) cardinal means the smaller distance, while the nice objects are those of lower cardinalities. Thus, the largest distance (the "coarsest" $\kappa^{-}$-shape) corresponds to $\kappa=\aleph_{0}$. The minimal nontrivial distance (the "finest" nontrivial $\kappa^{-}$-shape) corresponds 
to cardinality $\kappa$ of the object Clearly, if an object has cardinality $\kappa$, then, for every $\kappa^{\prime} \geq \kappa$, its $\kappa^{\prime}$-shape class becomes the category isomorphism class because, in that case, one is "in" the object.

Some interesting concrete categories $\mathcal{C}$ for considering the quotient shapes are as follows:

Set - sets and functions;

Ordg - partially ordered sets and genuine order-preserving functions;

$(U) P M e t$ - (ultra)pseudometric spaces with various kinds of morphisms; Top - topological spaces and mappings (and many of its subcategories); Unf - uniform spaces and uniformly continuous functions;

Mon - monoids and their homomorphisms;

Grp - groups and homomorphisms;

TGrp - topological groups and continuous homomorphisms;

Ring - rings and ring homomorphisms;

$R$-Mod (and Mod-R) - left (and right) $R$-modules and $R$-module homomorphisms, as well as many of its important subcategories;

$V e c t_{F}$ - vectorial spaces (over a fixed field $F$ ) and their linear functions.

In each of them, except Set and $V e c t_{F}$ (with the algebraic structure only), we have found examples which show that the quotient shape classifications are strictly coarser than the classification by isomorphisms.

Finally, the paper is written as a program (bringing only the main constructions and motivating examples in a few fairly known concrete categories), and the author expects an appropriate response of the specialists in the various concrete categories.

\section{PRELiminaries}

Let $\mathcal{S}$ denote the class of all sets. Recall ([10], III. 2) that a concrete category, denoted by $\mathcal{C}$, is a triple $(\mathcal{O}, U$, hom $)$, where

(i) $\mathcal{O}$ is a class whose members are called $\mathcal{C}$-objects;

(ii) $U: \mathcal{O} \rightarrow \mathcal{S}$ is a set-valued function, where, for each $\mathcal{C}$-object $X, U(X)$ is called the underlying set of $X$;

(iii) hom : $\mathcal{O} \times \mathcal{O} \rightarrow \mathcal{S}$ is a set-valued function, where, for each ordered pair $(X, Y)$ of $\mathcal{C}$-objects, $\operatorname{hom}(X, Y)$ is called the set of all $\mathcal{C}$-morphisms with domain $X$ and codomain $Y$;

such the following conditions are satisfied:

(1) for each ordered pair $(X, Y)$ of $\mathcal{C}$-objects, $\operatorname{hom}(X, Y)$ is a subset of the set $U(Y)^{U(X)}$ of all functions from $U(X)$ to $U(Y)$;

(2) for each $\mathcal{C}$-object $X$, the identity function $1_{U(X)}$ on the set $U(X)$ is a member of $\operatorname{hom}(X, X)$;

(3) for each triple $(X, Y, Z)$ of $\mathcal{C}$-objects, $f \in \operatorname{hom}(X, Y)$ and $g \in$ $\operatorname{hom}(Y, Z)$ implies that the function composite $g f \in \operatorname{hom}(X, Z)$. 
For instance, Set (the category of all sets and functions), Grp (the category of all groups and homomorphisms), Top (the category of all topological spaces and mappings - continuous functions) are concrete categories, while HTop (the category of all topological spaces and homotopy classes of mappings) is not a concrete category (proven by P. Freyd; see [10], III.4.7, p. 25). Roughly speaking, a concrete category $\mathcal{C}$ have the objects $(X, \sigma)$, where $X$ is a set and $\sigma$ is a structure, and the morphisms $f:(X, \sigma) \rightarrow(Y, \tau)$, where $f$ is a function of $X$ to $Y$ which preserves the structures by a given rule. Further, the function $U$ turns into so called forgetful functor $U: \mathcal{C} \rightarrow \operatorname{Set}, U(X, \sigma)=X$ and $U(f)=f$, that is faithful.

One usually denotes $\mathcal{O}$ by $O b(\mathcal{C})$ and $\operatorname{hom}(X, Y)$ by $\mathcal{C}(X, Y)$, while the union of all $\operatorname{hom}(X, Y)$ of $\mathcal{C}$ one denotes by $\operatorname{Mor}(\mathcal{C})$. Further, an element $f \in \mathcal{C}(X, Y)$ one often writes down as $f: X \rightarrow Y$.

According to [10], a congruence (or natural equivalence relation) on a category $\mathcal{C}$ (not necessarily concrete) is an equivalence relation $\sim$ on the class $\operatorname{Mor}(\mathcal{C})$ satisfying the next two conditions:

(a) every equivalence class by $\sim$ is contained in a unique $\mathcal{C}(X, Y)$;

(b) if $f \sim f^{\prime}$ and $g \sim g^{\prime}$, then $g f \sim g^{\prime} f^{\prime}$, whenever the compositions are meaningful.

A natural equivalence relation $\sim$ on $\mathcal{C}$ makes the composition of equivalence classes possible by putting $[g][f]=[g f]$. Consequently, there exists the category having the object class $O b(\mathcal{C})$, while the morphisms are the equivalence classes of those from $\mathcal{C}$ by $\sim$. It is called the quotient category of $\mathcal{C}$ with respect to $\sim$, and is denoted by $\mathcal{C} / \sim$.

We assume that the notion of a functor of a category $\mathcal{C}$ to a category $\mathcal{D}$, $F: \mathcal{C} \rightarrow \mathcal{D}$, is well known.

Further, we recall some basic notions and facts about a pro-category, as well as about an expansion and shape, by following [13]. A preordered set is an ordered pair $(\Lambda, \leq)$ consisting of a set $\Lambda$ and a preordering $\leq$ (reflexive and transitive). A preordered set $(\Lambda, \leq)$ is said to be directed provided

$$
\left(\forall \lambda, \lambda^{\prime} \in \Lambda\right)\left(\exists \lambda^{\prime \prime} \in \Lambda\right) \lambda, \lambda^{\prime} \leq \lambda^{\prime \prime} .
$$

Let $\mathcal{C}$ be a category. An inverse system, denoted by $\boldsymbol{X}$, in the category $\mathcal{C}$ is a triple $\left(\left(X_{\lambda}\right),\left(p_{\lambda \lambda^{\prime}}\right),(\Lambda, \leq)\right)$, abbreviated to $\left(X_{\lambda}, p_{\lambda \lambda^{\prime}}, \Lambda\right)$, where

- $(\Lambda, \leq)$ is a directed set, called the index set;

- for each $\lambda \in \Lambda, X_{\lambda}$ is a $\mathcal{C}$-object, called the term;

- for every related pair $\lambda \leq \lambda^{\prime}$ in $\Lambda, p_{\lambda \lambda^{\prime}}: X_{\lambda^{\prime}} \rightarrow X_{\lambda}$ is a $\mathcal{C}$-morphism, called the bonding morphism, such that $p_{\lambda \lambda}=1_{X_{\lambda}}\left(\equiv 1_{\lambda}\right)$ and $p_{\lambda \lambda^{\prime}} p_{\lambda^{\prime} \lambda^{\prime \prime}}=p_{\lambda \lambda^{\prime \prime}}$ whenever $\lambda \leq \lambda^{\prime} \leq \lambda^{\prime \prime}$.

In the case of $(\Lambda, \leq)=(\mathbb{N}, \leq)$ (the natural numbers), an inverse system $\boldsymbol{X}=\left(X_{i}, p_{i i^{\prime}}, \mathbb{N}\right)$ is called an inverse sequence. An inverse system indexed by a singleton is called a rudimentary (or constant) system and is denoted by 
$\lfloor X\rfloor$ where $X$ is the only term. We shall often abbreviate (not identify; see [17], Section 2) $\lfloor X\rfloor$ to $X$.

A morphism of inverse systems, denoted by

$$
\left(f_{\mu}, \phi\right): \boldsymbol{X} \rightarrow \boldsymbol{Y}=\left(Y_{\mu}, q_{\mu \mu^{\prime}}, M\right),
$$

consists of a function $\phi: M \rightarrow \Lambda$ and of $\mathcal{C}$-morphisms $f_{\mu}: X_{\phi(\mu)} \rightarrow Y_{\mu}$, for each $\mu \in M$, such that

$$
\left(\forall \mu \leq \mu^{\prime}\right)\left(\exists \lambda \geq \phi(\mu), \phi\left(\mu^{\prime}\right)\right) \quad f_{\mu} p_{\phi(\mu) \lambda}=q_{\mu \mu^{\prime}} f_{\mu^{\prime}} p_{\phi\left(\mu^{\prime}\right) \lambda} .
$$

The composition of morphisms of inverse systems, $\left(f_{\mu}, \phi\right): \boldsymbol{X} \rightarrow \boldsymbol{Y}$ and $\left(g_{\nu}, \psi\right): \boldsymbol{Y} \rightarrow \boldsymbol{Z}=\left(Z_{\nu}, r_{\nu \nu^{\prime}}, N\right)$, is well defined by putting

$$
\left(g_{\nu}, \psi\right)\left(f_{\mu}, \phi\right)=\left(h_{\nu}, \chi\right): \boldsymbol{X} \rightarrow \boldsymbol{Z}
$$

where

$$
\chi=\phi \psi: N \rightarrow \Lambda \quad \text { and } \quad h_{\nu}=g_{\nu} f_{\psi(\nu)}: X_{\chi(\nu)} \rightarrow Z_{\nu} .
$$

The composition is associative, and the identity morphism on an inverse system $\boldsymbol{X}$ is $\left(1_{\lambda}, 1_{\Lambda}\right): \boldsymbol{X} \rightarrow \boldsymbol{X}$. In this way is constructed a certain category, denoted by inv-C , which is called the inv-category of the category $\mathcal{C}$. Further, on each morphism set $i n v-\mathcal{C}(\boldsymbol{X}, \boldsymbol{Y})$, an equivalence relation $\sim$ is defined as follows:

$$
\left(f_{\mu}, \phi\right) \sim\left(f_{\mu}^{\prime}, \phi^{\prime}\right) \Leftrightarrow\left((\forall \mu \in M)\left(\exists \lambda \geq \phi(\mu), \phi^{\prime}(\mu)\right) f_{\mu} p_{\phi(\mu) \lambda}=f_{\mu}^{\prime} p_{\phi^{\prime}(\mu) \lambda}\right) .
$$

It is a natural equivalence relation on $i n v-\mathcal{C}$. The corresponding quotient category $($ inv- $\mathcal{C}) / \sim$ is denoted by pro- $\mathcal{C}$ and is called the pro-category of the category $\mathcal{C}$.

There exists a functor from $\mathcal{C}$ to pro- $\mathcal{C}$ determined by

$$
(f: X \rightarrow Y) \mapsto(\lfloor f\rfloor:\lfloor X\rfloor \rightarrow\lfloor Y\rfloor)
$$

(the rudimentary embedding). Although the brackets "[.」" are often dropped, one must not assume that $\mathcal{C}$ is a subcategory of pro-C (see [17], Section 2).

Let $X \in O b(\mathcal{C})$, let $\boldsymbol{X} \in O b($ pro-C) and let $\boldsymbol{p}:\lfloor X\rfloor \rightarrow \boldsymbol{X}$ be a morphism of pro-C. Then, $\boldsymbol{p}$ is said to be an inverse limit of $\boldsymbol{X}$ (in the category $\mathcal{C}$ ) if, for every morphism $\boldsymbol{f}:\lfloor Y\rfloor \rightarrow \boldsymbol{X}$ (of pro- $\mathcal{C}$ ), there exists a unique morphism $f: Y \rightarrow X$ (of $\mathcal{C}$ ) such that $\boldsymbol{p}\lfloor f\rfloor=\boldsymbol{f}$. Further, given a full subcategory $\mathcal{D} \subseteq \mathcal{C}, \boldsymbol{p}$ is said to be a $\mathcal{C}$-expansion of $X$ with respect to $\mathcal{D}$ if, for every

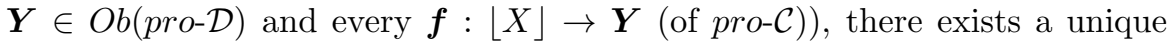
morphism $\boldsymbol{g}: \boldsymbol{X} \rightarrow \boldsymbol{Y}$ (of pro-C) satisfying $\boldsymbol{g} \boldsymbol{p}=\boldsymbol{f}$. If, in addition, $\boldsymbol{X} \in$ $O b($ pro- $\mathcal{D})$, then $\boldsymbol{p}$ is, for short, said to be a $\mathcal{D}$-expansion of $X$ (with respect to $\mathcal{D}$ itself). In that case, obviously, $\boldsymbol{g}$ belongs to pro- $\mathcal{D}$. The following two conditions characterize a $\mathcal{C}$-expansion (D-expansion) $\boldsymbol{p}:\lfloor X\rfloor \rightarrow \boldsymbol{X}$ :

(E1) $(\forall P \in O b(\mathcal{D}))(\forall h: X \rightarrow P$ of $\mathcal{C})(\exists \lambda \in \Lambda)\left(\exists f: X_{\lambda} \rightarrow P\right.$ of $\mathcal{C}($ of $\left.\mathcal{D})\right)$ $f p_{\lambda}=h$;

(E2) $\left(\forall f, f^{\prime}: X_{\lambda} \rightarrow P\right.$ of $\mathcal{C}($ of $\left.\mathcal{D})\right)\left(f p_{\lambda}=f^{\prime} p_{\lambda} \Rightarrow\left(\left(\exists \lambda^{\prime} \geq \lambda\right) f p_{\lambda \lambda^{\prime}}=\right.\right.$ $\left.f^{\prime} p_{\lambda \lambda^{\prime}}\right)$. 
Every two $\mathcal{D}$-expansions of the same $\mathcal{C}$-object are naturally isomorphic (as objects of pro- $\mathcal{D})$. If every $\mathcal{C}$-object $X$ admits a $\mathcal{D}$-expansion $\boldsymbol{p}:\lfloor X\rfloor \rightarrow \boldsymbol{X}$, then $\mathcal{D}$ is said to be dense in $\mathcal{C}$. Since [15, Section 3.3], the term "dense" may be replaced by the equivalent (more categorical) term $\pi$ pro-reflective. We shall use it in the sequel.

Given a category pair $(\mathcal{C}, \mathcal{D})$ such that $\mathcal{D} \subseteq \mathcal{C}$ is pro-reflective, $\mathcal{D}$ expansions $\boldsymbol{p}:\lfloor X\rfloor \rightarrow \boldsymbol{X}, \boldsymbol{p}^{\prime}:\lfloor X\rfloor \rightarrow \boldsymbol{X}^{\prime}, \boldsymbol{q}:\lfloor Y\rfloor \rightarrow \boldsymbol{Y}, \boldsymbol{q}^{\prime}:\lfloor Y\rfloor \rightarrow \boldsymbol{Y}^{\prime}$, and morphisms $\boldsymbol{f}: \boldsymbol{X} \rightarrow \boldsymbol{Y}, \boldsymbol{f}^{\prime}: \boldsymbol{X}^{\prime} \rightarrow \boldsymbol{Y}^{\prime}$, let us consider the following diagram

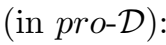

$$
\begin{array}{ccc}
\boldsymbol{X} & \stackrel{\boldsymbol{i}}{\rightarrow} & \boldsymbol{X}^{\prime} \\
\boldsymbol{f} \downarrow & & \downarrow \boldsymbol{f}^{\prime} \\
\boldsymbol{Y} & \rightarrow & \boldsymbol{Y}^{\prime}
\end{array},
$$

where $\boldsymbol{i}$ and $\boldsymbol{j}$ are the natural isomorphisms. We say that $\boldsymbol{f}$ is equivalent to $\boldsymbol{f}^{\prime}, \boldsymbol{f} \sim \boldsymbol{f}^{\prime}$, provided the above diagram commutes. The relation $\sim$ is a natural equivalence relation on pro- $\mathcal{D}$, and the equivalence class of an $f$ is denoted by $\langle\boldsymbol{f}\rangle$. Now the shape category for $(\mathcal{C}, \mathcal{D})$, denoted by $S h_{(\mathcal{C}, \mathcal{D})}$, is defined as follows:

$$
\begin{aligned}
& O b\left(S h_{(\mathcal{C}, \mathcal{D})}\right)=\operatorname{Ob}(\mathcal{C}) \\
& \operatorname{Sh}_{(\mathcal{C}, \mathcal{D})}(X, Y) \approx(\text { pro- } \mathcal{D})(\boldsymbol{X}, \boldsymbol{Y}),
\end{aligned}
$$

where $\boldsymbol{X}, \boldsymbol{Y}$ is any pair of the appropriate $\mathcal{D}$-expansions, i.e., a shape morphism $F: X \rightarrow Y$ is the equivalence class $\langle\boldsymbol{f}\rangle$ of a morphism $\boldsymbol{f} \in$ (pro$\mathcal{D})(\boldsymbol{X}, \boldsymbol{Y})$. Thus, such an $F$ is represented by a diagram

$$
\begin{array}{ccc}
\boldsymbol{X} & \boldsymbol{p} & X \\
\boldsymbol{f} \downarrow & & \\
\boldsymbol{Y} & \overleftarrow{q} & Y
\end{array} .
$$

The composition is well defined by means of any pair of the representatives, and the identity $1_{X}$ is defined by any $\mathbf{1}_{X}$. Given $X, Y \in O b(\mathcal{C})$, we say that $X$ and $Y$ have the same shape (type), $S h(X)=S h(Y)$, provided they are isomorphic objects in $S h_{(\mathcal{C}, \mathcal{D})}$. There also exists a functor $S: \mathcal{C} \rightarrow S h_{(\mathcal{C}, \mathcal{D})}$, called the shape functor, defined by $S(X)=X$ and $S(f)=F$, where $F=\langle\boldsymbol{f}\rangle$ and $\boldsymbol{f}$ is induced by $f([13$, I.2.3]).

Finally, all necessary facts concerning ordinals and cardinals one can find in [5], Chapter II. In addition, one can straightforwardly prove that, for every infinite set $X\left(|X| \geq \aleph_{0}\right)$ and every cardinal $\kappa$, the following facts hold true:

(e) if $\kappa<|X|$, then the set of all subsets $A \subseteq X$ having cardinality $|A|=\kappa$ has cardinality $|X|$;

(f) consequently, the set of all subsets $A \subseteq X$ having cardinalities $|A|<$ $|X|$ has cardinality $|X|$

(g) the set of all subsets $A \subseteq X$ having cardinality $|A|=|X|$ has cardinality $\left|2^{X}\right|=2^{|X|}$. 


\section{The Shapes In A CONCRETE CATEgOry - Generally}

Concerning a shape of objects, in general, one has to decide which ones are "nice" absolutely and/or relatively (with respect to a chosen one). In this approach, the main principle is:

In general, an object is "nice" if it is a quotient object belonging to a special full subcategory and if it (its "basis") has cardinality less than (less or equal to) a given infinite cardinal.

Given a category pair $(\mathcal{C}, \mathcal{D})$, where $\mathcal{D} \subseteq \mathcal{C}$ is full, and a cardinal $\kappa$, let $\mathcal{D}_{\kappa^{-}}\left(\mathcal{D}_{\kappa}\right)$ denote the full subcategory of $\mathcal{D}$ determined by all the objects having cardinalities or, in some special cases, the cardinalities of "bases" less than (less or equal to) $\kappa$.

By following the principle, let $\left(\mathcal{C}, \mathcal{D}_{\kappa^{-}}\right)\left(\left(\mathcal{C}, \mathcal{D}_{\kappa}\right)\right)$ be such a pair of concrete categories. If

(a) every $\mathcal{C}$-object $(X, \sigma)$ admits a directed set $R\left(X, \sigma, \kappa^{-}\right) \equiv \Lambda_{\kappa^{-}}$ $\left(R(X, \sigma, \kappa) \equiv \Lambda_{\kappa}\right)$ of equivalence relations $\lambda$ on $X$ such that each quotient object $\left(X / \lambda, \sigma_{\lambda}\right)$ has to belong to $\mathcal{D}_{\kappa^{-}}\left(\mathcal{D}_{\kappa}\right)$, while each quotient morphism $p_{\lambda}:(X, \sigma) \rightarrow\left(X / \lambda, \sigma_{\lambda}\right)$ has to belong to $\mathcal{C}$;

(b) the induced morphisms between quotient objects belong to $\mathcal{D}_{\kappa^{-}}\left(\mathcal{D}_{\kappa}\right)$;

(c) every morphism $f:(X, \sigma) \rightarrow(Y, \tau)$ of $\mathcal{C}$, having the codomain in $\mathcal{D}_{\kappa^{-}}$ $\left(\mathcal{D}_{\kappa}\right)$, factorizes uniquely through a quotient $p_{\lambda}:(X, \sigma) \rightarrow\left(X / \lambda, \sigma_{\lambda}\right)$, $f=g p_{\lambda}$, with $g$ belonging to $\mathcal{D}_{\kappa^{-}}\left(\mathcal{D}_{\kappa}\right)$,

then $\mathcal{D}_{\kappa^{-}}\left(\mathcal{D}_{\kappa}\right)$ is a pro-reflective subcategory of $\mathcal{C}$. Consequently, there exist a (nontrivial) "quotient" shape category $S h_{\left(\mathcal{C}, \mathcal{D}_{\kappa^{-}}\right)} \equiv S h_{\mathcal{D}_{\kappa^{-}}}(\mathcal{C}) \equiv S h_{\kappa^{-}}$ $\left(S h_{\left(\mathcal{C}, \mathcal{D}_{\kappa}\right)} \equiv S h_{\mathcal{D}_{\kappa}}(\mathcal{C}) \equiv S h_{\kappa}\right)$ obtained by the general construction described in Section 2.

Therefore, a $\kappa^{-}$-shape morphism $F_{\kappa^{-}}:(X, \sigma) \rightarrow(Y, \tau)$ is represented by a diagram (in pro-C)

$$
\begin{array}{ccc}
(\boldsymbol{X}, \boldsymbol{\sigma})_{\kappa^{-}} & \stackrel{p_{\kappa^{-}}}{\longleftarrow} & (X, \sigma) \\
\boldsymbol{f}_{\kappa^{-}} \downarrow & & \\
(\boldsymbol{Y}, \boldsymbol{\tau})_{\kappa^{-}} & \stackrel{q_{\kappa^{-}}}{\longleftarrow} & (Y, \tau)
\end{array}
$$

(with $\boldsymbol{p}_{\kappa^{-}}$and $\boldsymbol{q}_{\kappa^{-}}$- a pair of appropriate expansions), and similarly for a $\kappa$-shape morphism $F_{\kappa}:(X, \sigma) \rightarrow(Y, \tau)$. Since all $\mathcal{D}_{\kappa^{-}}$-expansions $\left(\mathcal{D}_{\kappa^{-}}\right.$ expansions) of a $\mathcal{C}$-object are mutually isomorphic objects of pro- $\mathcal{D}_{\kappa^{-}}$(pro$\mathcal{D}_{\kappa}$ ), the composition and identities follow straightforwardly (observe that every quotient morphism $p_{\lambda}$ is an effective epimorphism $\left(U\left(p_{\lambda}\right)\right.$ is a surjection), and thus condition (E2) follows trivially).

The corresponding "quotient" shape functors $S_{\kappa^{-}}: \mathcal{C} \rightarrow S h_{\left(\mathcal{C}, \mathcal{D}_{\kappa^{-}}\right)}$and $S_{\kappa}: \mathcal{C} \rightarrow S h_{\left(\mathcal{C}, \mathcal{D}_{\kappa}\right)}$ are defined in the same general manner. That means,

$$
S_{\kappa^{-}}(X, \sigma)=S_{\kappa}(X, \sigma)=(X, \sigma)
$$


if $f:(X, \sigma) \rightarrow(Y, \tau)$ is a $\mathcal{C}$-morphism, then, for every $\mu \in M_{\kappa^{-}}$, the composite $g_{\mu} f:(Y, \tau) \rightarrow\left(Y_{\mu}, \tau_{\mu}\right)$ factorizes (uniquely) through a $p_{\lambda(\mu)}:(X, \sigma) \rightarrow\left(X_{\lambda(\mu)}, \sigma_{\lambda(\mu)}\right)$, and thus, the correspondence $\mu \mapsto \lambda(\mu)$ yields a function $\varphi: M_{\kappa^{-}} \rightarrow \Lambda_{\kappa^{-}}$and a family of $\mathcal{D}_{\kappa^{-}}$-morphisms $f_{\mu}$ : $\left(X_{\varphi(\mu)}, \sigma_{\varphi(\mu)}\right) \rightarrow\left(Y_{\mu}, \tau_{\mu}\right)$ such that $q_{\mu} f=f_{\mu} p_{\varphi(\mu)}$; one easily shows that $\left(\varphi, f_{\mu}\right):(\boldsymbol{X}, \boldsymbol{\sigma})_{\kappa^{-}} \rightarrow(\boldsymbol{Y}, \boldsymbol{\tau})_{\kappa^{-}}$is a morphism of inv- $\mathcal{D}_{\kappa^{-}}$, so the equivalence class $\boldsymbol{f}_{\kappa^{-}}=\left[\left(\varphi, f_{\mu}\right)\right]:(\boldsymbol{X}, \boldsymbol{\sigma})_{\kappa^{-}} \rightarrow(\boldsymbol{Y}, \boldsymbol{\tau})_{\kappa^{-}}$is a morphism of pro- $\mathcal{D}_{\kappa^{-}}$; then we put $S_{\kappa^{-}}(f)=\left\langle\boldsymbol{f}_{\kappa^{-}}\right\rangle \equiv F_{\kappa^{-}}:(X, \sigma) \rightarrow(Y, \tau)$ in $S h_{\left(\mathcal{C}, \mathcal{D}_{\kappa^{-}}\right)}$. The identities and composition are obviously preserved. In the same way one defines the functor $S_{\kappa}$.

Furthermore, since $(\boldsymbol{X}, \boldsymbol{\sigma})_{\kappa^{-}}$is a subsystem of $(\boldsymbol{X}, \boldsymbol{\sigma})_{\kappa}$, one easily shows that there exists a functor $S_{\kappa^{-} \kappa}: S h_{\left(\mathcal{C}, \mathcal{D}_{\kappa}\right)} \rightarrow S h_{\left(\mathcal{C}, \mathcal{D}_{\kappa^{-}}\right)}$such that $S_{\kappa^{-} \kappa} S_{\kappa}=$ $S_{\kappa^{-}}$, i.e., the diagram

$$
S h_{\mathcal{D}_{\kappa^{-}}}(\mathcal{C})^{\swarrow S_{\kappa^{-}}} \stackrel{\mathcal{C}}{S_{\kappa^{-} \kappa}} S_{\kappa} \searrow \quad S h_{\mathcal{D}_{\kappa}}(\mathcal{C})
$$

commutes (compare the proof of Theorem 1). Moreover, an analogous functor $S_{\kappa \kappa^{\prime}}: S h_{\mathcal{D}_{\kappa^{\prime}}}(\mathcal{C}) \rightarrow S h_{\mathcal{D}_{\kappa}}(\mathcal{C})$, satisfying $S_{\kappa \kappa^{\prime}} S_{\kappa^{\prime}}=S_{\kappa}$, exists for every pair of infinite cardinals $\kappa \leq \kappa^{\prime}$.

Generally, in the case of $\kappa=\aleph_{0}$, the $\kappa^{-}$-shape is said to be the finite (quotient) shape, because all the objects in the expansions are of finite (bases) cardinalities, and is denoted by $S h\left(\mathcal{C}, \mathcal{D}_{\underline{0}}\right) \equiv S h_{\underline{0}}$.

REMARK 3.1. For every concrete category $\mathcal{C}$ such that $U[O b(\mathcal{C})]=$ $O b($ Set $)$, and every subcategory $\mathcal{B} \subseteq$ Set such that $O b(\mathcal{B})=O b($ Set $)$ and $M$ or $\mathcal{B} \supseteq \operatorname{Mor} \mathcal{C}$, there exists a unique category $\mathcal{C}_{\mathcal{B}} \supseteq \mathcal{C}$ that it enlarges $\mathcal{C}$ by $\operatorname{Mor} \mathcal{C}_{\mathcal{B}}=\operatorname{Mor} \mathcal{B}$ (the new morphisms are all functions of $\mathcal{B}$ - ignoring the structure). The maximal such enlargement $\overline{\mathcal{C}}$ occurs in the case $\mathcal{B}=\operatorname{Set}$. The only condition for the existence of a $\kappa$-shape theory for $\mathcal{C}_{\mathcal{B}}$ is that there exist quotient objects belonging to $\mathcal{C}$. Then, clearly, for every infinite cardinal $\kappa$, "to be of the same $\kappa$-shape" in $\mathcal{C}_{\mathcal{B}}$ is equivalent to "to be mutually equipotent as sets" ("to have equipotent bases"), that does not imply "to be isomorphic in $\mathcal{C}$ ". Therefore, the notion of $\kappa$-shape makes sense for every category $\mathcal{C}_{\mathcal{B}}$ as well. However, in this paper, we will not consider the $\kappa$-shapes of any such $\mathcal{C}_{\mathcal{B}}$

Clearly, there are concrete categories (mostly some special subcategories of a concrete category) that do not admit a (nontrivial quotient) shape theory in the above sense. In the sequel, we shall apply the above procedure to the following well known concrete categories:

- Set - sets and functions;

- Ordg - partially ordered sets and genuine order-preserving functions; 
- PMet (UPMet) - pseudometric (ultrapseudometric) spaces with several classes of functions;

- Top - topological spaces and mappings (only, in general);

- Mon - monoids and their homomorphisms;

- Grp - groups and homomorphisms (and $A b \subseteq G r p$ - abelian groups);

- Ring - rings and ring homomorphisms;

- $R$-Mod - (left) $R$-modules and $R$-module homomorphisms;

- Vect $F$ - vectorial spaces (over a field $F$ ) with the algebraic structure (only) and their linear functions.

\section{ThE SHAPES OF SETS}

We shall begin with the category Set of all sets and functions in order to demonstrate the technique. Since there is no structure in this case, one should expect that all the shape classifications of sets and equipotency coincide, that is, indeed, the case.

The construction by quotients of any $\left(\kappa^{-}\right)$shape category for the category Set serves as the general pattern for any concrete category. We follow the main principle of our "philosophy": every finite set is "nice", and, if $X$ is infinite, every set $Y$ with $|Y|<|X|$ is "nice with respect to $X$ ".

Let $X$ be a set, and let $\Lambda \equiv \Lambda(X)$ be the set of all equivalence relations $\sim \equiv \subseteq X \times X$ on $X$. Put

$$
\lambda \leq \lambda^{\prime} \Leftrightarrow(\forall x \in X)[x]_{\lambda^{\prime}} \subseteq[x]_{\lambda}
$$

(the refinement), where $[x]_{\lambda}$ and $[x]_{\lambda^{\prime}}$ are the equivalence classes of $x$ by $\lambda$ and by $\lambda^{\prime}$, respectively. Then $(\Lambda, \leq)$ becomes a poset (partially ordered set) that is directed (it is closed under intersections and $\lambda, \lambda^{\prime} \leq \lambda \cap \lambda^{\prime}$ ). Given a $\lambda \in \Lambda$, denote by $X_{\lambda}$ the quotient set $X / \lambda$ and by $p_{\lambda}: X \rightarrow X_{\lambda}$ the quotient function $x \mapsto[x]_{\lambda}$. If $\lambda \leq \lambda^{\prime}$, then there exists a unique function $p_{\lambda \lambda^{\prime}}: X_{\lambda^{\prime}} \rightarrow X_{\lambda}$ such that $p_{\lambda \lambda^{\prime}} p_{\lambda^{\prime}}=p_{\lambda}$. Further, if $\lambda \leq \lambda^{\prime} \leq \lambda^{\prime \prime}$, then $p_{\lambda \lambda^{\prime}} p_{\lambda^{\prime} \lambda^{\prime \prime}}=p_{\lambda \lambda^{\prime \prime}}$. Moreover, all the functions $p_{\lambda}$ and $p_{\lambda \lambda^{\prime}}$ are surjective. In this way we have obtained the inverse system $\boldsymbol{X}=\left(X_{\lambda}, p_{\lambda \lambda^{\prime}}, \Lambda\right)$ in Set (an object of pro-Set) and the morphism

$$
\boldsymbol{p}=\left(p_{\lambda}\right): X \rightarrow \boldsymbol{X}
$$

of pro-Set, where $X$ is identified with the rudimentary system $\lfloor X\rfloor$. It is obvious that $\boldsymbol{p}: X \rightarrow \boldsymbol{X}$ is the limit (of $\boldsymbol{X}$ ) and an expansion (of $X$ ) in Set (with respect to any subcategory). Namely, $(\Lambda, \leq$ ) has the maximal element which corresponds to the finest equivalence relation on $X$ - the diagonal $\Delta_{X}$. Therefore, $X_{\Delta_{X}}=X$ and $p_{\Delta_{X}}=1_{X}$, so the conclusion follows. This, obviously, is not very useful for our aim. Thus we need to remove $\Delta_{X}$ from $\Lambda$. It is clear that the poset $\left(\Lambda \backslash\left\{\Delta_{X}\right\}, \leq\right)$ might not be directed any more. If $X$ is an infinite set and $\Delta_{X}$ is removed from $\Lambda$, then in order to assure the needed directedness one has, in general, to remove from $\Lambda$ a "lot" of other 
equivalence relations. Consequently, the resulting morphism

$$
\boldsymbol{p}^{\prime}=\left(p_{\lambda}\right): X \rightarrow \boldsymbol{X}^{\prime}=\left(X_{\lambda}, p_{\lambda \lambda^{\prime}}, \Lambda^{\prime}\right)
$$

is not, generally, a limit (of $\boldsymbol{X}^{\prime}$ ) nor an expansion (of $X$ ) (of course, the system $\boldsymbol{X}^{\prime}=\left(X_{\lambda}, p_{\lambda \lambda^{\prime}}, \Lambda^{\prime}\right)$ has a limit $\boldsymbol{p}^{\prime}=\left(p_{\lambda}^{\prime}\right): X^{\prime} \rightarrow \boldsymbol{X}^{\prime}$, such that, in general, $X^{\prime}$ is not equipotent to $X)$. Nevertheless, for every infinite set $X$, it is possible to choose a directed subset of $\Lambda^{\prime} \subset \Lambda \backslash\left\{\Delta_{X}\right\}$ so that the restricted morphism $\boldsymbol{p}^{\prime}$ is an expansion.

Given an infinite cardinal $\kappa\left(\geq \aleph_{0}\right)$, let $\operatorname{Set}_{\kappa^{-}}\left(\right.$Set $\left._{\kappa}\right)$ denote the full subcategory of Set having the objects all the sets with cardinalities less than (less or equal to) $\kappa$. We are to prove that $S e t_{\kappa^{-}}$and $S e t_{\kappa}$ are pro-reflective subcategories of Set. More explicitly, the following lemma holds true.

Lemma 4.1. Let $\kappa$ be an infinite cardinal and let $X$ be a set. Let $\Lambda_{\kappa^{-}} \subseteq \Lambda$ $\left(\Lambda_{\kappa} \subseteq \Lambda\right)$ be the subset of all $\lambda \in \Lambda$ such that $\left|X_{\lambda}\right|<\kappa\left(\left|X_{\lambda}\right| \leq \kappa\right)$. Then the morphism

$$
\begin{gathered}
\boldsymbol{p}_{\kappa^{-}}=\left(p_{\lambda}\right): X \rightarrow \boldsymbol{X}_{\kappa^{-}}=\left(X_{\lambda}, p_{\lambda \lambda^{\prime}}, \Lambda_{\kappa^{-}}\right) \\
\left(\boldsymbol{p}_{\kappa}=\left(p_{\lambda}\right): X \rightarrow \boldsymbol{X}_{\kappa}=\left(X_{\lambda}, p_{\lambda \lambda^{\prime}}, \Lambda_{\kappa}\right)\right)
\end{gathered}
$$

of pro-Set is a Set $\kappa_{\kappa^{-}}$-expansion (Set $\kappa_{\kappa^{-}}$expansion) of $X$ having all $p_{\lambda \lambda^{\prime}}$ and all $p_{\lambda}$ epimorphisms (surjections in Set).

Proof. Firstly, $\Lambda_{\kappa^{-}}$and $\Lambda_{\kappa}$ are directed. Namely,

$$
\left|X_{\lambda \cap \lambda^{\prime}}\right| \leq\left|X_{\lambda}\right| \cdot\left|X_{\lambda^{\prime}}\right| \quad \text { and } \quad \kappa^{\prime} \cdot \kappa^{\prime \prime}<\kappa\left(\kappa^{\prime} \cdot \kappa^{\prime \prime} \leq \kappa\right)
$$

whenever $\kappa^{\prime}, \kappa^{\prime \prime}<\kappa\left(\kappa^{\prime}, \kappa^{\prime \prime} \leq \kappa\right)$ and $\kappa \geq \aleph_{0}$ (see [5], Section 8). Thus, $\boldsymbol{X}_{\kappa^{-}}$ $\left(\boldsymbol{X}_{\kappa}\right)$ is an inverse system in $\operatorname{Set}_{\kappa^{-}}\left(\right.$Set $\left._{\kappa}\right)$ and $\boldsymbol{p}_{\kappa^{-}}: X \rightarrow \boldsymbol{X}_{\kappa^{-}}\left(\boldsymbol{p}_{\kappa}: X \rightarrow\right.$ $\left.\boldsymbol{X}_{\kappa}\right)$ is a morphism of pro-Set. Now, if $|X|<\kappa$, the assertions are true by the consideration from above $\left(\Lambda_{\kappa^{-}}=\Lambda_{\kappa}=\Lambda\right.$, and there exists max $\left.\Lambda=\Delta_{X}\right)$. Let $|X|=\kappa$. Then $\Lambda_{\kappa}=\Lambda$ and, consequently, $\boldsymbol{p}_{\kappa}: X \rightarrow \boldsymbol{X}_{\kappa}$ is a Set ${ }_{\kappa}$-expansion) of $X$ as before. To verify that $\boldsymbol{p}_{\kappa^{-}}: X \rightarrow \boldsymbol{X}_{\kappa^{-}}$is a Set $_{\kappa^{-}}$-expansion of $X$, consider any function $f: X \rightarrow Y$, where $|Y|<\kappa$. It implies that the fibers $f^{-1}[\{y\}], y \in Y$, of $f$ induce the partition of $X$. i.e., a unique equivalence relation $\sim_{f}$ on $X$, such that $\left|X / \sim_{f}\right|<\kappa$. Thus, there exists a $\lambda \in \Lambda_{\kappa^{-}}$such that $\sim_{f}=\lambda$ and, consequently, $X / \sim_{f}=X_{\lambda}$. Therefore, $f$ factorizes through $p_{\lambda}$, i.e., there exists a function $g: X_{\lambda} \rightarrow Y, g p_{\lambda}=f$. Moreover, $g$ is unique since all $p_{\lambda}$ are epimorphisms (surjections). Finally, if $|X|>\kappa$, the proof holds in the same manner.

The above constructed expansions $\boldsymbol{p}_{\kappa^{-}}: X \rightarrow \boldsymbol{X}_{\kappa^{-}}$and $\boldsymbol{p}_{\kappa}: X \rightarrow \boldsymbol{X}_{\kappa}$ of $X$ are said to be canonical. In the case of $\kappa=\aleph_{0}$, we shall, in the sequel, Set ${\aleph_{0}^{-}}$denote by $S e t_{\underline{0}}$, and $\boldsymbol{p}_{\aleph_{0}^{-}}=\left(p_{\lambda}\right): X \rightarrow \boldsymbol{X}_{\aleph_{0}^{-}}=\left(X_{\lambda}, p_{\lambda \lambda^{\prime}}, \Lambda_{\aleph_{0}^{-}}\right)$by

$$
\boldsymbol{p}_{\underline{0}}=\left(p_{\lambda}\right): X \rightarrow \boldsymbol{X}_{\underline{0}}=\left(X_{\lambda}, p_{\lambda \lambda^{\prime}}, \Lambda_{\underline{0}}\right),
$$

which is a cofinite expansion of $X$ in terms of finite sets. Clearly, if $X$ is a finite set, then one may reduce $\boldsymbol{p}_{\underline{0}}$, as well as every $\boldsymbol{p}_{\kappa^{-}}$and $\boldsymbol{p}_{\kappa}$, to the 
identity $1_{X}$. And, in general, if $\kappa \geq|X|(\kappa>|X|)$, one may take $\boldsymbol{p}_{\kappa}=1_{X}$ $\left(\boldsymbol{p}_{\kappa^{-}}=\boldsymbol{p}_{\kappa}=1_{X}\right)$.

REMARK 4.2. By accepting the generalized continuum hypothesis (see [5], II. Sections 6 and 7), if $\kappa=\aleph_{\alpha} \geq \aleph_{0}$ is an accessible cardinal (i.e., $\alpha$ is not a limit ordinal), then $\kappa^{-}=\aleph_{\alpha-1}$, while, if $\kappa$ is inaccessible and $\alpha=\beta+\omega$ ( $\beta$ is a limit ordinal and $\omega$ is the first limit ordinal), then $\kappa^{-}$may represent the well-ordered set $\left\{\aleph \mid \aleph_{\beta}<\aleph<\aleph_{\beta+\omega}\right\}=\left\{\aleph_{\beta+n} \mid n \in \mathbb{N}\right\}$.

By following the general procedure of constructing of a shape category (Section 2), one establishes the next theorem.

TheOREM 4.3. For each infinite cardinal $\kappa$, there exist two shape categories $S h_{\kappa^{-}}($Set $)$and $S h_{\kappa}($ Set $)$ of sets having for the object classes $O b(S e t)$, while

and

$$
S h_{\kappa^{-}}(\operatorname{Set})(X, Y) \approx \operatorname{pro-Set}_{\kappa^{-}}\left(\boldsymbol{X}_{\kappa^{-}}, \boldsymbol{Y}_{\kappa^{-}}\right)
$$

$$
\operatorname{Sh}_{\kappa}(\operatorname{Set})(X, Y) \approx \operatorname{pro-Set}_{\kappa}\left(\boldsymbol{X}_{\kappa}, \boldsymbol{Y}_{\kappa}\right),
$$

where $\boldsymbol{X}_{\kappa^{-}}, \boldsymbol{Y}_{\kappa^{-}}$and $\boldsymbol{X}_{\kappa}, \boldsymbol{Y}_{\kappa}$ are the systems in any pair of appropriate expansions of $X, Y$ respectively. Further, there exist two shape functors

$$
S_{\kappa^{-}}: S e t \rightarrow S h_{\kappa^{-}}(\text {Set }) \quad \text { and } \quad S_{\kappa}: S e t \rightarrow S h_{\kappa}(\text { Set })
$$

keeping the objects fixed, and there exists a unique relating functor

$$
S_{\kappa^{-} \kappa}: S h_{\kappa}(S e t) \rightarrow S h_{\kappa^{-}}(S e t)
$$

keeping the objects fixed such that $S_{\kappa^{-} \kappa_{\kappa}} S_{\kappa}=S_{\kappa^{-}}$. Moreover, for every related pair $\kappa \leq \kappa^{\prime}$ (including the case $\kappa \leq \kappa^{\prime-}$ whenever it occurs), there exists the relating functor $S_{\kappa \kappa^{\prime}}: S h_{\kappa^{\prime}}(S e t) \rightarrow S h_{\kappa}($ Set $)$ such that $S_{\kappa \kappa^{\prime}} S_{\kappa^{\prime}}=S_{\kappa}$.

Proof. We only want to demonstrate how one constructs $S_{\kappa^{-}}(f)$, and then to prove the existence of $S_{\kappa^{-} \kappa}$. Let $\kappa$ be an infinite cardinal and let $f: X \rightarrow Y$ be a function. Let $\boldsymbol{p}_{\kappa^{-}}=\left(p_{\lambda}\right): X \rightarrow \boldsymbol{X}_{\kappa^{-}}=\left(X_{\lambda}, p_{\lambda \lambda^{\prime}}, \Lambda_{\kappa^{-}}\right)$and $\boldsymbol{q}_{\kappa^{-}}=\left(q_{\mu}\right): Y \rightarrow \boldsymbol{Y}_{\kappa^{-}}=\left(Y_{\mu}, q_{\mu \mu^{\prime}}, M_{\kappa^{-}}\right)$be the canonical Set $_{\kappa^{-}}$-expansions of $X$ and $Y$ respectively. If $|X|<\kappa$, then there exists $\lambda_{*}=\max \Lambda_{\kappa^{-}}$with $p_{\lambda_{*}}=1_{X}$. Thus, $f$ yields $\boldsymbol{f}=\boldsymbol{q}_{\kappa^{-}} f=\left[\left(c_{\lambda_{*}}, q_{\mu} f\right)\right]: \boldsymbol{X}_{\kappa^{-}} \rightarrow \boldsymbol{Y}_{\kappa^{-}}$of pro-Set $_{\kappa^{-}}$ such that $\boldsymbol{f} \boldsymbol{p}_{\kappa^{-}}=\boldsymbol{q}_{\kappa^{-}} f$. Then $S(f): X \rightarrow Y$ of $S h_{\kappa^{-}}($Set $)$is the equivalence class $\langle\boldsymbol{f}\rangle$ of $\boldsymbol{f}$ in pro-Set $_{\kappa^{-}}$. Let $|X| \geq \kappa$. Since, for each $\mu \in M,\left|Y_{\mu}\right|<\kappa$, the function $q_{\mu} f: X \rightarrow Y_{\mu}$ admits (as in the proof of Lemma 1) a $\varphi(\mu) \in \Lambda$ and a unique function $f_{\mu}: X_{\varphi(\mu)} \rightarrow Y_{\mu}$ such that $f_{\mu} p_{\varphi(\mu)}=q_{\mu} f$. Further, if $\mu \leq \mu^{\prime}$, then there exists a $\lambda \geq \varphi(\mu), \varphi\left(\mu^{\prime}\right)$ such that $f_{\mu} p_{\varphi(\mu) \lambda}=q_{\mu \mu^{\prime}} f_{\mu^{\prime}} p_{\varphi\left(\mu^{\prime}\right) \lambda}$. In that way $f$ yields an $\boldsymbol{f}=\left[\left(\varphi, f_{\mu}\right)\right]: \boldsymbol{X}_{\kappa^{-}} \rightarrow \boldsymbol{Y}_{\kappa^{-}}$of pro-Set $_{\kappa^{-}}$such that $\boldsymbol{f} \boldsymbol{p}_{\kappa^{-}}=\boldsymbol{q}_{\kappa^{-}} f$. Then $S(f): X \rightarrow Y$ of $S h_{\kappa^{-}}($Set $)$is the equivalence class $\langle\boldsymbol{f}\rangle$ of $\boldsymbol{f}$ in pro-Set $_{\kappa^{-}}$.

In order to prove the existence of $S_{\kappa^{-} \kappa}$, let $F_{\kappa} \in S h_{\kappa}(X, Y)$ and let an $\boldsymbol{f}_{\kappa}: \boldsymbol{X}_{\kappa} \rightarrow \boldsymbol{Y}_{\kappa}$ be its representative with $\boldsymbol{X}_{\kappa}$ and $\boldsymbol{Y}_{\kappa}$ canonical. Recall 
that the canonical inverse system $\boldsymbol{X}_{\kappa^{-}}$and $\boldsymbol{Y}_{\kappa^{-}}$are subsystems of $\boldsymbol{X}_{\kappa}$ and $\boldsymbol{Y}_{\kappa}$, respectively, and that $\boldsymbol{X}_{\kappa}$ and $\boldsymbol{Y}_{\kappa}$ are subobjects of $\boldsymbol{X}_{\kappa^{-}}$and $\boldsymbol{Y}_{\kappa^{-}}$, respectively, in pro-Set $_{\kappa} \subseteq$ pro-Set. (In the categorical sense, every inverse system is the subobject of a chosen subsystem!) Let $\boldsymbol{j}_{X}: \boldsymbol{X}_{\kappa} \rightarrow \boldsymbol{X}_{\kappa^{-}}$and $\boldsymbol{j}_{Y}: \boldsymbol{Y}_{\kappa} \rightarrow \boldsymbol{Y}_{\kappa^{-}}$. be the corresponding subobject (mono)morphisms, i.e., $\boldsymbol{j}_{X}=\left[\left(j, 1_{\lambda}\right)\right]$, where $j: \Lambda_{\kappa^{-}} \hookrightarrow \Lambda_{\kappa}$ is the inclusion and $1_{\lambda}$ is the identity on $X_{\lambda}, \lambda \in \Lambda_{\kappa^{-}}$, and $j_{Y}=\left[\left(j^{\prime}, 1_{\mu}\right)\right]$, where $j^{\prime}: M_{\kappa^{-}} \hookrightarrow M_{\kappa}$ is the inclusion and $1_{\mu}$ is the identity on $Y_{\mu}, \mu \in M_{\kappa^{-}}$Notice that $\boldsymbol{j}_{X} \boldsymbol{p}_{\kappa}=\boldsymbol{p}_{\kappa^{-}}$and $\boldsymbol{j}_{Y} \boldsymbol{q}_{\kappa}=\boldsymbol{q}_{\kappa^{-}}$. Let us consider the morphism $\boldsymbol{j}_{Y} \boldsymbol{f}_{\kappa} \boldsymbol{p}_{\kappa}: X \rightarrow \boldsymbol{Y}_{\kappa^{-}}$. Since $\boldsymbol{p}_{\kappa^{-}}: X \rightarrow \boldsymbol{X}_{\kappa^{-}}$is a Set $_{\kappa^{-}}$expansion of $X$, there exists a unique $\boldsymbol{f}_{\kappa^{-}}: \boldsymbol{X}_{\kappa^{-}} \rightarrow \boldsymbol{Y}_{\kappa^{-}}$such that $\boldsymbol{f}_{\kappa^{-}} \boldsymbol{j}_{X} \boldsymbol{p}_{\kappa}=\boldsymbol{f}_{\kappa^{-}} \boldsymbol{p}_{\kappa^{-}}=\boldsymbol{j}_{Y} \boldsymbol{f}_{\kappa} \boldsymbol{p}_{\kappa}$. Denote $F_{\kappa^{-}}=\left\langle\boldsymbol{f}_{\kappa^{-}}\right\rangle \in S h_{\kappa^{-}}(X, Y)$, and put $S_{\kappa^{-} \kappa}\left(F_{\kappa}\right)=F_{\kappa^{-}}$. Finally, observe that $\boldsymbol{f}_{\kappa^{-}} \boldsymbol{j}_{X}=\boldsymbol{j}_{Y} \boldsymbol{f}_{\kappa}$ (because $\boldsymbol{p}_{\kappa}$ is an epimorphism, Section 3). Now one straightforwardly verifies that $S_{\kappa^{-} \kappa}$ is a functor. The commutativity of functors follows by the construction.

Corollary 4.4. All the shape categories of Set make an "inverse system"

$$
S h_{\underline{Q}}(S e t) \leftarrow \cdots \leftarrow S h_{\kappa^{-}}(S e t) \leftarrow S h_{\kappa}(\text { Set }) \leftarrow \cdots
$$

in CAT (quasicategory of all categories and functors) over the well-ordered index class $K^{-} \cup K$, where $K$ is the class of all cardinals, while $K^{-}$is the class of all $\kappa^{-}$representing the well ordered sets $\left\{\aleph_{\beta+n} \mid \beta\right.$ - a limit ordinal, $n \in \mathbb{N}\}$ (see Remark 2).

REMARK 4.5. Clearly, for every infinite cardinal $\kappa$, every function $f$ : $X \rightarrow Y$ yields the shape morphisms $S_{\kappa^{-}}(f)$ and $S_{\kappa}(f)$ (i.e., pro-morphisms $\boldsymbol{f}_{\kappa^{-}}$and $\boldsymbol{f}_{\kappa}$ ). However, the $\kappa^{-}$-converse ( $\kappa$-converse) does not hold whenever $\kappa$ is not sufficiently large. More precisely, given a morphism $\boldsymbol{f}: \boldsymbol{X}_{\kappa} \rightarrow \boldsymbol{Y}_{\kappa}$ of pro-Set $_{\kappa}$, let us consider a diagram

$$
\begin{aligned}
& \lim \boldsymbol{X}_{\kappa} \leftarrow X \\
& \downarrow \lim \boldsymbol{f} \\
& \lim \boldsymbol{Y}_{\kappa} \leftarrow Y
\end{aligned}
$$

Now, if $\Delta_{Y} \notin M_{\kappa}$, the injective function $Y \rightarrow \lim \boldsymbol{Y}_{\kappa}$, in general, is not surjective. Hence, $(\lim \boldsymbol{f})[X]$ might be not a subset of $Y$.

Although there exist the various (quotient) shapes $S h_{\kappa^{-}}(X)$ and $S h_{\kappa}(X)$ of a set $X$ and the shape morphism sets are richer than the function sets, all the (quotient) shape classifications on Set coincide with the equipotence. It is not surprising because the only condition in the construction is to take care about cardinalities. The main step in proving this fact is the next lemma.

LEMMA 4.6. Let $X$ and $Y$ be infinite sets such that $|X| \neq|Y|$. Then, for every infinite cardinal $\kappa, S h_{\kappa^{-}}(X) \neq S h_{\kappa^{-}}(Y)$ and $S h_{\kappa}(X) \neq S h_{\kappa}(Y)$. 
Proof. Without loss of generality, we may assume that $\aleph_{0} \leq \kappa_{1}=|X|<$ $|Y|=\kappa_{2}$. The relevant possibilities are as follows: (a) $\kappa>\kappa_{2}$; (b) $\kappa=\kappa_{2}$; (c) $\kappa_{1}<\kappa<\kappa_{2} ;$ (d) $\kappa=\kappa_{1} ;(\mathrm{e}) \kappa<\kappa_{1}$.

(a) The both sets $X$ and $Y$ represent their shape $\kappa^{-}$-classes and shape $\kappa$-classes. The conclusion follows.

(b) $X$ represents its shape $\kappa^{-}$-class and shape $\kappa$-class, and $Y$ represents its shape $\kappa$-class, while $\boldsymbol{Y}_{\kappa^{-}}$(of the canonical Set $_{\kappa^{-}}$-expansion of $Y$ ) is not isomorphic to any rudimentary system. The conclusion follows.

(c) $X$ represents its shape $\kappa^{-}$-class and shape $\kappa$-class, while $\boldsymbol{Y}_{\kappa^{-}}$and $\boldsymbol{Y}_{\kappa}$ are not isomorphic to rudimentary systems. The conclusion follows.

(d) $X$ represents its shape $\kappa$-class, while $\boldsymbol{X}_{\kappa^{-}}, \boldsymbol{Y}_{\kappa^{-}}$and $\boldsymbol{Y}_{\kappa}$ are not isomorphic to rudimentary systems. Thus, it suffices to prove that the canonical systems $\boldsymbol{X}_{\kappa^{-}}$and $\boldsymbol{Y}_{\kappa^{-}}$are not isomorphic objects of pro-Set $\kappa_{\kappa^{-}}$. In order to do it, notice that $\lambda \in \Lambda_{0}$, i.e., $\left|X_{\lambda}\right|<\aleph_{0}$, implies that there exists an $x \in X$ such that $\left|[x]_{\lambda}\right|=|X|$. Hence, in general, since $\Lambda_{0} \subseteq \Lambda_{\kappa^{-}} \subseteq \Lambda_{\kappa} \subseteq \Lambda \subseteq 2^{X \times X}$, the fact $(\mathrm{g})$ at the end of Section 2 implies that

$$
2^{|X|}=\left|\Lambda_{0}\right| \leq\left|\Lambda_{\kappa^{-}}\right| \leq\left|\Lambda_{\kappa}\right| \leq|\Lambda| \leq\left|2^{X \times X}\right|=2^{|X \times X|}=2^{|X|} .
$$

Therefore, all the $\Lambda_{\kappa^{-}}$and $\Lambda_{\kappa}$ are of the same cardinality - $2^{|X|}$. Especially, in this case, $\left|\Lambda_{\kappa^{-}}\right|=2^{\kappa_{1}}<2^{\kappa_{2}}=\left|M_{\kappa^{-}}\right|$. Without loss of generality, we may pass to the associated cofinite systems $\boldsymbol{X}_{\kappa^{-}}^{\prime}=\left(X_{\bar{\lambda}}^{\prime}, p_{\bar{\lambda} \bar{\lambda}^{\prime}}^{\prime}, \bar{\Lambda}_{\kappa^{-}}\right)$and $\boldsymbol{Y}_{\kappa^{-}}^{\prime}=\left(Y_{\bar{\mu}}^{\prime}, q_{\bar{\mu} \bar{\mu}}^{\prime}, M_{\kappa^{-}}\right)$([13], I.1.2, Theorem 2). Recall that $\left|\bar{\Lambda}_{\kappa^{-}}\right|=\left|\Lambda_{\kappa^{-}}\right|$ and $\left|M_{\kappa^{-}}\right|=\left|M_{\kappa^{-}}\right|$.

Let us assume to the contrary, i.e., that $\boldsymbol{X}_{\kappa^{-}}^{\prime} \cong \boldsymbol{Y}_{\kappa^{-}}^{\prime}$ in pro- Set $_{\kappa^{-}}$. Let $\boldsymbol{f}: \boldsymbol{X}_{\kappa^{-}}^{\prime} \rightarrow \boldsymbol{Y}_{\kappa^{-}}^{\prime}$ be an isomorphism. Choose a special representative $\left(\varphi, f_{\bar{\mu}}\right)$ of $\boldsymbol{f}$ (having the index function $\varphi$ increasing, [13], I. 1.2, Lemma 2). Then, for every related pair $\bar{\mu} \leq \bar{\mu}^{\prime}$,

$$
f_{\mu} p_{\varphi(\bar{\mu}) \varphi\left(\bar{\mu}^{\prime}\right)}^{\prime}=q_{\bar{\mu} \bar{\mu}^{\prime}}^{\prime} f_{\bar{\mu}^{\prime}} .
$$

Similarly, there exists a special representative $\left(\psi, g_{\bar{\lambda}}\right)$ of $\boldsymbol{f}^{-1}: \boldsymbol{Y}_{\kappa^{-}} \rightarrow \boldsymbol{X}_{\kappa^{-}}$ ( $\psi$ is increasing) such that, for every related pair $\bar{\lambda} \leq \bar{\lambda}^{\prime}$,

$$
g_{\bar{\lambda}} q_{\psi(\bar{\lambda}) \psi\left(\bar{\lambda}^{\prime \prime}\right)}^{\prime}=p_{\bar{\lambda} \bar{\lambda}^{\prime}}^{\prime} g_{\bar{\mu}^{\prime}}
$$

Since

$$
\left(\varphi, f_{\bar{\mu}}\right) \circ\left(\psi, g_{\bar{\lambda}}\right)=\left(\psi \varphi, f_{\bar{\mu}} g_{\varphi(\bar{\mu})}\right) \sim\left(1_{M_{\kappa^{-}}}, 1_{Y_{\bar{\mu}}^{\prime}}\right),
$$

one infers that, for every $\bar{\mu} \in M_{\kappa^{-}}$, there exists a $\bar{\mu}^{\prime} \geq \bar{\mu}, \psi \varphi(\bar{\mu})$ such that

$$
f_{\bar{\mu}} g_{\varphi(\bar{\mu})} q_{\psi \varphi(\bar{\mu}) \bar{\mu}^{\prime}}^{\prime}=q_{\bar{\mu} \bar{\mu}^{\prime}}^{\prime}
$$

Because of the choice of the special representatives $\left(q_{\bar{\mu} \bar{\mu}^{\prime}}^{\prime}=q_{\bar{\mu} \psi \varphi(\bar{\mu})}^{\prime} q_{\psi \varphi(\bar{\mu}) \bar{\mu}^{\prime}}^{\prime}\right)$ and the surjectivity of all $q_{\mu \mu^{\prime}}$, and thus of all $q_{\bar{\mu}_{1} \bar{\mu}_{2}}^{\prime}$, it follows that

$$
\left(\forall \bar{\mu} \in M_{\kappa^{-}}\right) \quad f_{\bar{\mu}} g_{\varphi(\bar{\mu})}=q_{\bar{\mu} \psi \varphi(\mu)}^{\prime} .
$$


Now, since

$$
\left|M_{\kappa^{-}}\right|=\left|M_{\kappa^{-}}\right|=\kappa_{2}>\kappa_{1}=\left|\Lambda_{\kappa^{-}}\right|=\left|\bar{\Lambda}_{\kappa^{-}}\right| \geq \aleph_{0},
$$

there exists a $\bar{\lambda}_{0} \in \bar{\Lambda}_{\kappa^{-}}$and there exists infinitely many elements $\bar{\mu} \in M_{\kappa^{-}}$ such that $\varphi(\bar{\mu})=\bar{\lambda}_{0}$. Put $\bar{\mu}_{0}=\psi\left(\bar{\lambda}_{0}\right) \in M_{\kappa^{-}}$. Then,

$$
\bar{\mu}_{0}=\psi \varphi(\bar{\mu}) \geq \bar{\mu}
$$

for infinitely many $\bar{\mu} \in M_{\kappa^{-}}$. It follows that $\left(M_{\kappa^{-}}, \leq^{\prime}\right)$ is not cofinite - a contradiction.

(e) In this case any of $\boldsymbol{X}_{\kappa^{-}}, \boldsymbol{X}_{\kappa}, \boldsymbol{Y}_{\kappa^{-}}, \boldsymbol{Y}_{\kappa}$ is not isomorphic to a rudimentary system. To prove that $\boldsymbol{X}_{\kappa^{-}}$and $\boldsymbol{Y}_{\kappa^{-}}$are not mutually isomorphic in pro-Set $_{\kappa^{-}}$, and that $\boldsymbol{X}_{\kappa}$ and $\boldsymbol{Y}_{\kappa}$ are not mutually isomorphic in pro-Set $_{\kappa}$ is quite similar to the proof of case $(\mathrm{d})$.

Theorem 4.7. For every pair $X, Y$ of sets and every infinite cardinal $\kappa$,

$$
S h_{\kappa^{-}}(X)=S h_{\kappa^{-}}(Y) \Leftrightarrow|X|=|Y| \Leftrightarrow S h_{\kappa}(X)=S h_{\kappa}(Y) .
$$

Proof. If $|X|=|Y|$, then the implications follow by functoriality. We have to prove the converses. Let, firstly, $S h_{\kappa^{-}}(X)=S h_{\kappa^{-}}(Y)$. If $X$ and $Y$ are finite sets, then $|X|=|Y|$ because, for every $\kappa$, the representatives of their $\kappa^{-}$-shape classes are $X$ and $Y$ themselves respectively. If $X$ is finite and $Y$ infinite, then $X$ represents every of its $\kappa^{-}$-shape classes, and $Y$ represents every of its $\kappa^{-}$-shape classes whenever $\kappa>|Y|$, while $\boldsymbol{Y}_{\kappa^{-}}$is not isomorphic to any rudimentary system whenever $\kappa \leq|Y|$. Therefore, $S h_{\kappa^{-}}(X) \neq S h_{\kappa^{-}}(Y)$ - a contradiction. If $X$ is infinite and $Y$ is finite, the same conclusion follows. Thus both $X$ and $Y$ are either finite (the previous case) or infinite. Then must be, by Lemma $2,|X|=|Y|$. The proof of the $\kappa$-case is quite similar.

In the pointed case, i.e., for category $S t_{*}$ of all pointed sets $\left(X, x_{0}\right)$ and the base point-preserving functions $f:\left(X, x_{0}\right) \rightarrow\left(Y, y_{0}\right)$, the equivalence relations should be compatible with morphisms, i.e.,

$$
x \sim x_{0} \Leftrightarrow f(x) \sim^{\prime} f\left(x_{0}\right)=y_{0} .
$$

Since this compatibility is preserved under intersections, the construction for Set remains valid for $S e t_{*}$ as well. Moreover, since the proof of Lemma 2 works in the pointed case as well, the pointed analogue of Theorem 2 holds true. Therefore, all the shapes of a pointed set reduce to its (pointed) equipotency class.

\section{THE SHAPES OF ORDERED SETS}

Firstly, we have to select a certain class of function between ordered sets to be the needed morphisms. Let $(X, \leq)$ and $\left(Y, \leq^{\prime}\right)$ be posets (partially ordered sets) and let $f: X \rightarrow Y$ be a function. If, for every pair $x_{1}, x_{2} \in X$, the implication 
(1) $x_{1}<x_{2} \Rightarrow f\left(x_{1}\right) \leq \prime f\left(x_{2}\right)$

holds, then $f:(X, \leq) \rightarrow\left(Y, \leq^{\prime}\right)$ is said to be order-preserving. If the condition

(2) $f\left(x_{1}\right)<^{\prime} f\left(x_{2}\right) \Rightarrow x_{1} \leq x_{2}$

is fulfilled, then $f:(X, \leq) \rightarrow\left(Y, \leq^{\prime}\right)$ is said to be conversely order-preserving. (As usually, " $<$ " means " $\leq$ " and " $\neq$ ", and thus, $x_{1} \leq x_{2}$ in (2) is equivalent to $x_{1}<x_{2}$.) If $f$ satisfies both conditions (1) and (2), then $f$ is said to be genuine order-preserving. Observe that every constant function is genuine order-preserving: Further, if $(X, \leq)$ is totally ordered, then, for every poset $\left(Y, \leq^{\prime}\right)$, every order-preserving function $f:(X, \leq) \rightarrow\left(Y, \leq^{\prime}\right)$ is genuine orderpreserving.

EXAmPle 5.1. (a) Let $\left(\mathbb{N}, \leq^{*}\right)$ be the set of natural numbers with the ordering $\leq^{*}$,

$$
\left(\forall n_{1}, n_{2} \in \mathbb{N}\right) n_{1} \leq^{*} n_{2} \Leftrightarrow \frac{n_{2}}{n_{1}} \in \mathbb{N},
$$

and let $(\mathbb{N}, \leq)$ be ordered as usual. Then the identity function $1_{\mathbb{N}}$ of $\left(\mathbb{N}, \leq^{*}\right)$ onto $(\mathbb{N}, \leq)$ has property (1) but not $(2)$, while conversely, $1_{\mathbb{N}}$ of $(\mathbb{N}, \leq)$ onto $\left(\mathbb{N}, \leq^{*}\right)$ has property $(2)$ but not (1). An order-preserving (and thus, genuine) function $f:(\mathbb{N}, \leq) \rightarrow\left(\mathbb{N}, \leq^{*}\right)$ is, for instance, $f(n)=n$ !. Conversely, every non-constant genuine order-preserving function $g:\left(\mathbb{N}, \leq^{*}\right) \rightarrow(\mathbb{N}, \leq)$ has to be constant on $\mathbb{N} \backslash\{1\}$ (see also Example 6 (a) at the end of this section). For instance,

$$
g(n)=\left\{\begin{array}{c}
k, n=1 \\
k+l, n \neq 1
\end{array} .\right.
$$

(b) Let $\left(\mathbb{Z}, \leq^{\prime}\right)$ be the set of integers with the ordering defined by the arrows as below

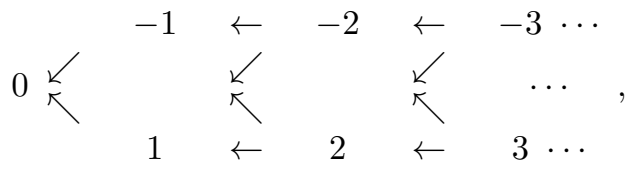

(the slanting arrows mean that $n<^{\prime}-(n+1)$ and $-n<^{\prime} n+1, n \in \mathbb{N}$, i.e., $\left.m_{1}<^{\prime} m_{2} \Leftrightarrow\left|m_{1}\right|<\left|m_{2}\right|, m \in \mathbb{Z}\right)$ and let $(\{0\} \cup \mathbb{N}, \leq)$ be the set of nonnegative integers with the standard ordering. Let $f: \mathbb{Z} \rightarrow\{0\} \cup \mathbb{N}$ be defined by $f(0)=0$ and by $f(-n)=f(n)=n, n \in \mathbb{N}$, and let $g: \mathbb{Z} \rightarrow$ $\{0\} \cup \mathbb{N}$ be defined by $g(0)=0$ and by $g(-n)=n, g(n)=n+1, n \in \mathbb{N}$. Then $f:\left(\mathbb{Z}, \leq^{\prime}\right) \rightarrow(\{0\} \cup \mathbb{N}, \leq)$ is a genuine order-preserving function, while $g:\left(\mathbb{Z}, \leq^{\prime}\right) \rightarrow(\{0\} \cup \mathbb{N}, \leq)$ is an order-preserving function which is not genuine order-preserving. Namely, $g(-n)=n<n+1=g(n)$, while $-n$ and $n$ are not related in $\left(\mathbb{Z}, \leq^{\prime}\right)$.

All the posets and all the (conversely, genuine) order-preserving functions make a category, denoted by $\operatorname{Ord}(\operatorname{Ordc}, \mathrm{Ordg})$, and $\operatorname{Ordg} \subseteq$ Ord $\cap \operatorname{Ordc}$ having the same object class. Namely, for every $(X, \leq)$, the identity function 
$1_{X}$ is obviously genuine order-preserving, while, given an $f:(X, \leq) \rightarrow\left(Y, \leq^{\prime}\right)$ and a $g:\left(Y, \leq^{\prime}\right) \rightarrow\left(Z, \leq^{\prime \prime}\right)$, that are (conversely, genuine) order-preserving, then, for every pair $x_{1}, x_{2} \in X$,

$$
\begin{aligned}
& x_{1}<x_{2} \Rightarrow f\left(x_{1}\right) \leq^{\prime} f\left(x_{2}\right) \Rightarrow g f\left(x_{1}\right) \leq^{\prime \prime} g f\left(x_{2}\right) \\
& \left(g f\left(x_{1}\right)<^{\prime \prime} g f\left(x_{2} \Rightarrow f\left(x_{1}\right)<^{\prime} f\left(x_{2}\right) \Rightarrow x_{1}<x_{2}\right) .\right.
\end{aligned}
$$

Therefore, $g f$ is also (conversely, genuine) order preserving.

Secondly, we have to select those equivalence relations on $X$ that are compatible with their quotient functions on the corresponding posets. In order to do it, given a poset $(X, \leq)$ and an equivalence relation $\lambda$ on $X$, let us define

$$
\left[x_{1}\right]_{\lambda} \leq\left[x_{2}\right]_{\lambda} \Leftrightarrow\left(\left[x_{1}\right]_{\lambda}=\left[x_{2}\right]_{\lambda} \vee\left[x_{1}\right]_{\lambda}<\left[x_{2}\right]_{\lambda}\right),
$$

where " $<$ " refers to all the elements, i.e., for every $x_{1}^{\prime} \in\left[x_{1}\right]_{\lambda}$ and every $x_{2}^{\prime} \in$ $\left[x_{2}\right]_{\lambda}$, it holds $x_{1}^{\prime}<x_{2}^{\prime}$. Then the quotient set $X / \lambda$ becomes the (canonical) poset $(X / \lambda, \leq)$. However, the quotient function $p: X \rightarrow X / \lambda$ is not, in general, (the given) order-preserving. For our purpose, a congruence on a poset $(X, \leq)$ is needed, i.e., an equivalence relation $\lambda \equiv \sim$ on $X$ such that, for all $x_{1}, x_{2}, x_{1}^{\prime}, x_{2}^{\prime} \in X$, the implication

$$
\left(x_{1} \leq x_{2} \wedge x_{1} \nsim x_{2} \wedge x_{1} \sim x_{1}^{\prime} \wedge x_{2} \sim x_{2}^{\prime}\right) \Rightarrow x_{1}^{\prime} \leq x_{2}^{\prime}
$$

holds ( $\wedge$ " denotes the logic conjunction). The diagonal $\Delta_{X}$ is obviously the finest congruence on $(X, \leq)$. Every congruence $\lambda$ assures that $x_{1}<x_{2}$ and $\left[x_{1}\right]_{\lambda} \neq\left[x_{2}\right]_{\lambda}$ imply $\left[x_{1}\right]_{\lambda}<\left[x_{2}\right]_{\lambda}$. Also, if $(X, \leq)$ is totally (well-) ordered, then so is $(X / \lambda, \leq)$. Notice that, for a given congruence $\lambda$ on a poset $(X, \leq)$ and an $x \in X$, the congruence class $[x]_{\lambda}$ may contain some mutually unrelated elements (see Example 2 below).

ExAmPLE 5.2. Let $\left(\mathbb{Z}, \leq^{\prime}\right)$ be the poset of Example $1(\mathrm{~b})$. Then the partition $\{0\},-\mathbb{N}, \mathbb{N}$ of $\mathbb{Z}$ yields a unique equivalence relation $\lambda$ on $\mathbb{Z}$ such that

$$
\left(\mathbb{Z} / \lambda, \leq^{\prime}\right)=\left(\{\{0\},-\mathbb{N}, \mathbb{N}\}, \leq^{\prime}\right) \equiv\left(\{[0],[-1],[1]\}, \leq^{\prime}\right)
$$

and $[0]<[-1],[0]<[1]$, while $[-1]$ and [1] are not related $(-1$ and 1 are not related in $\left.\left(\mathbb{Z}, \leq^{\prime}\right)\right)$. Obviously, $\lambda$ is not a congruence on $\left(\mathbb{Z}, \leq^{\prime}\right)(-1<2$, while $[-1]$ and $[2]=[1]$ are not related in $\left.\left(\mathbb{Z} / \lambda, \leq^{\prime}\right)\right)$. Consequently, the quotient function $p: \mathbb{Z} \rightarrow \mathbb{Z} / \lambda$ is not (the) order preserving. On the other hand, for each $n \in \mathbb{N}$, the partition $\{0\},\{-1,1, \cdots,-\mathrm{n}, \mathrm{n}\},\{-n-1, n+1, \cdots\}$ of $\mathbb{Z}$ yields a unique equivalence relation $\lambda_{n}$ on $\mathbb{Z}$ such that

$$
\left(\mathbb{Z} / \lambda_{n}, \leq^{\prime}\right)=\left(\{[0],[1],[n+1]\}, \leq^{\prime}\right) .
$$

One easily verifies that $\lambda_{n}$ is a congruence on $\left(\mathbb{Z}, \leq^{\prime}\right)$ and the quotient function $p_{n}^{\prime}:\left(\mathbb{Z}, \leq^{\prime}\right) \rightarrow\left(\mathbb{Z} / \lambda_{n}, \leq^{\prime}\right)$ is genuine order-preserving. (Moreover, $\left(\mathbb{Z} / \cap \lambda_{n}, \leq^{\prime}\right.$ ) is well-ordered and isomorphic to $(\mathbb{N}, \leq)$; see Example 3 below.) 
Let $X$ and $Y$ be sets and let $\lambda \equiv \sim$ and $\mu \equiv \sim^{\prime}$ be equivalence relations on $X$ and $Y$ respectively. A function $f: X \rightarrow Y$ is said to be $(\lambda, \mu)$-preserving if, for every pair $x, x^{\prime} \in X$, the implication

$$
x \sim x^{\prime} \Rightarrow f(x) \sim^{\prime} f\left(x^{\prime}\right)
$$

holds.

Lemma 5.3. (i) For every congruence $\lambda$ on a poset $(X, \leq)$, the quotient function $p:(X, \leq) \rightarrow(X / \lambda, \leq)$ is a genuine order-preserving surjection.

(ii) If, in addition, $\lambda^{\prime}$ is a finer congruence on $(X, \leq)$, then the induced function $\tilde{p}:\left(X / \lambda^{\prime}, \leq\right) \rightarrow(X / \lambda, \leq)$, satisfying $\tilde{p} p^{\prime}=p$, is genuine order-preserving as well.

(iii) If $f:(X, \leq) \rightarrow\left(Y, \leq^{\prime}\right)$ is a (conversely, genuine) order- and $(\lambda, \mu)$ preserving function, where $\lambda$ is a congruence on $(X, \leq)$ and $\mu$ is a congruence on $\left(Y, \leq^{\prime}\right)$, then the induced function $\tilde{f}:(X / \lambda, \leq) \rightarrow\left(Y / \mu, \leq^{\prime}\right.$ ), satisfying $\tilde{f} p=q f$, is (conversely, genuine) order-preserving.

Proof. Let $\lambda \equiv \sim$ be a congruence on a poset $(X, \leq)$, and let $p: X \rightarrow$ $X / \lambda, p(x)=[x]$, be the quotient function. Let $x_{1}, x_{2} \in X$ such that $x_{1}<x_{2}$. If $x_{1} \sim x_{2}$, then

$$
p\left(x_{1}\right)=\left[x_{1}\right]=\left[x_{2}\right]=p\left(x_{2}\right),
$$

and thus, $p\left(x_{1}\right) \leq p\left(x_{2}\right)$. If $x_{1} \nsim x_{2}$, then $x_{1}<x_{2}$ implies (by definitions),

$$
p\left(x_{1}\right)=\left[x_{1}\right]<\left[x_{2}\right]=p\left(x_{2}\right) \Rightarrow p\left(x_{1}\right) \leq p\left(x_{2}\right) .
$$

Further, if $x_{1}, x_{2} \in X$ such that $p\left(x_{1}\right)<p\left(x_{2}\right)$, i.e., $\left[x_{1}\right]<\left[x_{2}\right]$, then $x_{1}<x_{2}$ (by definition). Therefore, the quotient function $p:(X, \leq) \rightarrow(X / \lambda, \leq)$ is genuine order-preserving, and (i) is proven.

Given a congruence $\sim^{\prime} \equiv \lambda^{\prime} \geq \lambda$ (the refinement), the function $\tilde{p}: X / \lambda^{\prime} \rightarrow$ $X / \lambda$, satisfying $\tilde{p} p^{\prime}=p$, is well defined by $\tilde{p}\left([x]^{\prime}\right)=[x]$. Let $x_{1}, x_{2} \in X$ such that $\left[x_{1}\right]^{\prime}<\left[x_{2}\right]^{\prime}$ (in $\left(X / \lambda^{\prime}, \leq\right)$ ). Then, $x_{1}<x_{2}$ and, by (i),

$$
\tilde{p}\left(\left[x_{1}\right]^{\prime}\right)=\left[x_{1}\right]=p\left(x_{1}\right) \leq p\left(x_{2}\right)=\left[x_{2}\right]=\tilde{p}\left(\left[x_{2}\right]^{\prime}\right) .
$$

Further, if $x_{1}, x_{2} \in X$ such that $\tilde{p}\left(\left[x_{1}\right]^{\prime}\right)<\tilde{p}\left(\left[x_{2}\right]^{\prime}\right)$, then

$$
\left[x_{1}\right]=p\left(x_{1}\right)=\tilde{p}\left(\left[x_{1}\right]^{\prime}\right)<\tilde{p}\left(\left[x_{2}\right]^{\prime}\right)=p\left(x_{2}\right)=\left[x_{2}\right] .
$$

Since $\lambda^{\prime} \geq \lambda$, it follows that $\left[x_{1}\right]^{\prime}<\left[x_{2}\right]^{\prime}$ as well. Therefore, $\tilde{p}:\left(X / \lambda^{\prime}, \leq\right) \rightarrow$ $(X / \lambda, \leq)$ is a genuine order-preserving function, and (ii) is proven.

Let $f:(X, \leq) \rightarrow\left(Y, \leq^{\prime}\right)$ be an order- and $(\lambda, \mu)$-preserving function, where $\lambda$ is a congruence $\sim$ on $(X, \leq)$ and $\mu$ is a congruence $\sim^{\prime}$ on $\left(Y, \leq^{\prime}\right)$. Let $p:(X, \leq) \rightarrow(X / \lambda, \leq)$ and $q:\left(Y, \leq^{\prime}\right) \rightarrow\left(Y / \mu, \leq^{\prime}\right)$ be the corresponding quotient functions. Then $\tilde{f}: X / \lambda \rightarrow Y / \mu$ is well defined by putting $\tilde{f}([x])=$ $[f(x)]^{\prime}$. Thus, for every $x \in X$,

$$
\tilde{f} p(x)=\tilde{f}([x])=[f(x)]^{\prime}=q f(x) .
$$


Let $\left[x_{1}\right]<\left[x_{2}\right]$. Then $x_{1}<x_{2}$, and thus by (i), for the composite $q f(=\tilde{f} p)$,

$$
\tilde{f}\left(\left[x_{1}\right]\right)=\tilde{f} p\left(x_{1}\right)=q f\left(x_{1}\right) \leq^{\prime} q f\left(x_{2}\right)=\tilde{f} p\left(x_{2}\right)=\tilde{f}\left(\left[x_{2}\right]\right)
$$

holds. Hence, the induced function $\tilde{f}:(X / \lambda, \leq) \rightarrow\left(Y / \mu, \leq^{\prime}\right)$ is order-preserving. If, in addition, $f$ is conversely order-preserving, then (by (i))

$$
\begin{aligned}
\tilde{f}\left(\left[x_{1}\right]\right)<^{\prime} \tilde{f}\left(\left[x_{2}\right]\right) \Leftrightarrow\left[f\left(x_{1}\right)\right]^{\prime}<^{\prime}\left[f\left(x_{2}\right)\right]^{\prime} & \Rightarrow f\left(x_{1}\right)<^{\prime} f\left(x_{2}\right) \\
& \Rightarrow x_{1}<x_{2} \Rightarrow\left[x_{1}\right]<\left[x_{2}\right] .
\end{aligned}
$$

proving that $\tilde{f}$ is conversely order-preserving too. This proves (iii), and completes the proof of the lemma.

Notice that the intersection of two congruences $\lambda$ and $\lambda^{\prime}$ on a poset $(X, \leq)$ is again a congruence on $(X, \leq)$, finer than $\lambda$ and $\lambda^{\prime}$. The above proven facts (Lemma 3, (i) and (ii)) imply that one can associate with every poset $(X, \leq)$ a certain (canonical - by means of quotients) inverse system $(\boldsymbol{X}, \leq)=\left(\left(X_{\lambda}, \leq\right.\right.$ )$\left., p_{\lambda \lambda^{\prime}}, \Lambda\right)$ in $\operatorname{Ordg}($ Ordc, Ord $)$ and a (canonical) morphism $\boldsymbol{p}=\left(p_{\lambda}\right):(X, \leq$ )$\rightarrow(\boldsymbol{X}, \leq)$ of pro-Ordg (pro-Ordc, pro-Ord), where $\Lambda$ is the (index) set consisting of all congruences on $(X, \leq)$ ordered by $\lambda \leq \lambda^{\prime} \Leftrightarrow\left(\lambda^{\prime}\right.$ refines $\left.\lambda\right)$. Moreover, all $p_{\lambda}$ and $p_{\lambda \lambda^{\prime}}$ are (effective) epimorphisms. Since there exists $\max \Lambda$ (corresponding to $\Delta_{X}$ ), it is not satisfactory for our purpose, except $X$ is a finite set. However, as in the case of category Set, given an infinite cardinal $\kappa$, one may restrict his consideration to the subset $\Lambda_{\kappa^{-}}\left(\Lambda_{\kappa}\right)$ of $\Lambda$ such that each quotient set $X_{\lambda}$ has cardinality less than (less or equal to) $\kappa$. Still, to obtain an expansion, the genuine order-preserving functions are needed (see the proof of Lemma 4 below). Let us denote by $O r d g_{\kappa^{-}}\left(O r d g_{\kappa}\right)$ the full subcategory of $\operatorname{Ordg}$ having the objects all the posets $(X, \leq)$ of cardinalities less than (less or equal to) $\kappa$.

LEMMA 5.4. For every infinite cardinal $\kappa\left(\geq \aleph_{0}\right)$, the subcategories Ordg ${\kappa^{-}}$, Ordg $g_{\kappa} \subseteq$ Ordg are pro-reflective. Moreover, in both cases, each poset admits the canonical epimorphic (quotient) expansion.

Proof. We only have to verify condition (E1) for the canonical morphisms $\boldsymbol{p}_{\kappa^{-}}=\left(p_{\lambda}\right):(X, \leq) \rightarrow(\boldsymbol{X}, \leq)_{\kappa^{-}}$and $\boldsymbol{p}_{\kappa}=\left(p_{\lambda}\right):(X, \leq) \rightarrow(\boldsymbol{X}, \leq)_{\kappa}$ of pro-Ordg. (Condition (E2) follows trivially.) Let us consider the $\kappa$-case. Let a $\left(Y, \leq^{\prime}\right) \in O b\left(O r d g_{\kappa}\right)$ and an $f:(X, \leq) \rightarrow\left(Y, \leq^{\prime}\right)$ of $\operatorname{Mor}(O r d g)$ be given. If $|X| \leq \kappa$, then there exists $\max \Lambda_{\kappa} \equiv \lambda_{*}$ and $p_{\lambda_{*}}=1_{X}:\left(X, \leq_{\lambda_{*}}\right.$ )$\rightarrow(X, \leq)$, so that there is nothing to prove (a desired $g=f$ belonging to $\left.\operatorname{Mor}\left(\operatorname{Or}_{\bar{d}} g_{\kappa}\right)\right)$. Let $|X|>\kappa$. Since $|Y| \leq \kappa$, the fibers $f^{-1}[\{y\}], y \in Y$, make a partition of $X$, i.e., an equivalence relation $\sim_{f} \equiv \lambda_{f} \in X \times X$ such that $\left|X / \lambda_{f}\right| \leq \kappa$. Moreover, $\lambda_{f}$ is a congruence on $(X, \leq)$. Indeed, if $x_{1} \leq x_{2}$, $x_{1} \chi_{f} x_{2}$ (i.e., $x_{1}<x_{2}$ and $\left.f\left(x_{1}\right) \neq f\left(x_{2}\right)\right), x_{1}^{\prime} \sim_{f} x_{1}$ and $x_{2}^{\prime} \sim_{f} x_{2}$, then

$$
f\left(x_{1}^{\prime}\right)=f\left(x_{1}\right) \neq f\left(x_{2}\right)=f\left(x_{2}^{\prime}\right) .
$$


Since $f$ is order-preserving (and $x_{1} \chi_{f} x_{2}$ ), it follows that

$$
f\left(x_{1}^{\prime}\right)=f\left(x_{1}\right)<^{\prime} f\left(x_{2}\right)=f\left(x_{2}^{\prime}\right),
$$

and since $f$ is also conversely order-preserving, one finally infers that $x_{1}^{\prime}<x_{2}^{\prime}$, and thus, $x_{1}^{\prime} \leq x_{2}^{\prime}$. Therefore, there exists a $\lambda \in \Lambda_{\kappa}$ such that $p:(X, \leq) \rightarrow$ $\left(X / \lambda_{f}, \leq\right)$ is the appropriate $p_{\lambda}:(X . \leq) \rightarrow\left(X_{\lambda}, \leq\right)$ of $\boldsymbol{p}_{\kappa}$. Finally, by applying Lemma 3 (iii), a desired $g$ may be $\tilde{f}:\left(X_{\lambda}, \leq\right) \rightarrow\left(Y / \Delta_{Y}, \leq^{\prime}\right)=$ $\left(Y, \leq^{\prime}\right)$. The proof of the $\kappa^{-}$-case is quite similar.

Now, the general construction of a shape category and a shape functor yields the main fact (compare Theorem 1 and its proof).

THEOREM 5.5. For each infinite cardinal $\kappa$, there exist two shape categories $S h_{\kappa^{-}}(O r d g)$ and $S h_{\kappa}(O r d g)$ of partially ordered sets, both having the same object class $\mathrm{Ob}(\mathrm{Ord})$, while

$$
S h_{\kappa^{-}}(\operatorname{Ordg})\left((X, \leq),\left(Y, \leq^{\prime}\right)\right) \approx \operatorname{pro-Ordg}\left((\boldsymbol{X}, \leq)_{\kappa^{-}},\left(\boldsymbol{Y}, \leq^{\prime}\right)_{\kappa^{-}}\right)
$$

and

$$
\operatorname{Sh}_{\kappa}(\operatorname{Ordg})\left((X, \leq),\left(Y, \leq^{\prime}\right)\right) \approx \operatorname{pro-Ordg}\left((\boldsymbol{X}, \leq)_{\kappa},\left(\boldsymbol{Y}, \leq^{\prime}\right)_{\kappa}\right),
$$

where $(\boldsymbol{X}, \leq)_{\kappa^{-}},\left(\boldsymbol{Y}, \leq^{\prime}\right)_{\kappa^{-}}$and $(\boldsymbol{X}, \leq)_{\kappa},\left(\boldsymbol{Y}, \leq^{\prime}\right)_{\kappa}$ are the systems in any pair of appropriate expansions of $(X \leq),\left(Y, \leq^{\prime}\right)$ respectively.

Further, there exist two shape functors $S_{\kappa^{-}}:$Ordg $\rightarrow S h_{\kappa^{-}}($Ordg) and $S_{\kappa}: O r d g \rightarrow S h_{\kappa}(O r d g)$ keeping the objects fixed, and there exists a unique relating functor $S_{\kappa^{-} \kappa}: S h_{\kappa}(O r d g) \rightarrow S h_{\kappa^{-}}($Ordg) keeping the objects fixed, such that $S_{\kappa^{-}} S_{\kappa}=S_{\kappa^{-}}$. Moreover, for every related pair $\kappa \leq \kappa^{\prime}$ (including the case $\kappa \leq \kappa^{\prime-}$ whenever it occurs), there exists the relating functor $S_{\kappa \kappa^{\prime}}$ : $S h_{\kappa^{\prime}}($ Ordg $) \rightarrow S h_{\kappa}($ Ordg $)$ such that $S_{\kappa \kappa^{\prime}} S_{\kappa^{\prime}}=S_{\kappa}$.

EXAMPLE 5.6. It is obvious that the ordered sets $\left(\mathbb{Z}, \leq^{\prime}\right)$ and $(\{0\} \cup$ $\mathbb{N}, \leq$ ) of Example 1 are not isomorphic objects of Ordg. They also are not isomorphic to the standard ordered set $(\mathbb{Z}, \leq)$ of integers. We will prove that they all are of the same finite shape, i.e., that $S h_{\underline{0}}\left(\mathbb{Z}, \leq^{\prime}\right)=S h_{0}(\{0\} \cup \mathbb{N}, \leq$ )$=S h_{\underline{0}}(\mathbb{Z}, \leq)$. It suffices to verify that their canonical $O r d g_{0}$-expansions are isomorphic objects of pro-Ords 0 . Let

$$
\boldsymbol{p}_{\underline{0}}:(\{0\} \cup \mathbb{N}, \leq) \rightarrow(\boldsymbol{N}, \leq)_{\underline{0}} \quad \text { and } \quad \boldsymbol{q}_{\underline{0}}:\left(\mathbb{Z}, \leq^{\prime}\right) \rightarrow\left(\boldsymbol{W}, \leq^{\prime}\right)_{\underline{0}}
$$

be the canonical $\operatorname{Ordg}_{\underline{0}}$-expansions of $(\{0\} \cup \mathbb{N}, \leq)$ and $\left(\mathbb{Z}, \leq^{\prime}\right)$ respectively. In this case we shall show even more, that the inclusion

$$
j:(\{0\} \cup \mathbb{N}, \leq) \hookrightarrow\left(\mathbb{Z}, \leq^{\prime}\right)
$$

(which is a genuine order-preserving function) induces an isomorphism of $(\boldsymbol{N}, \leq)_{\underline{0}}$ to $\left(\boldsymbol{W}, \leq^{\prime}\right)_{\underline{0}}$, i.e., that

$$
\boldsymbol{j}_{\underline{0}}=\left[\left(\varphi, j_{\mu}\right)\right]:\left(\left(N_{\lambda}, \leq\right), p_{\lambda \lambda^{\prime}}, \Lambda_{\underline{0}}\right) \rightarrow\left(\left(W_{\mu}, \leq^{\prime}\right), q_{\mu \mu^{\prime}}, M_{\underline{0}}\right)
$$

is an isomorphism of pro-Ordg $g_{0}$. Firstly, notice (see Examples 1 and 2) that every congruence on $\left(\mathbb{Z}, \leq^{\prime}\right)$ yields a unique (by the restriction) congruence on 
$(\{0\} \cup \mathbb{N} ; \leq)$, and conversely, every congruence on $(\{0\} \cup \mathbb{N}, \leq)$ admits a unique congruence extension on $\left(\mathbb{Z}, \leq^{\prime}\right)$. Therefore, we may consider the index sets $\Lambda_{\underline{0}}$ and $M_{\underline{0}}$ to be equal. Further, each term $\left(N_{\lambda}, \leq\right), \lambda \in \Lambda_{\underline{0}}$, having $\left|N_{\lambda}\right| \geq 2$, is an ordered set of the form

$$
N_{\lambda}=\left\{\left[n_{1}^{\lambda}\right], \ldots,\left[n_{k+1}^{\lambda}\right]\right\}, k \in \mathbb{N}, n_{i}^{\lambda} \in\{0\} \cup \mathbb{N}, i \in\{1, \ldots, k+1\},
$$

where

$$
\begin{aligned}
& {\left[n_{1}^{\lambda}\right]=\left\{n \in\{0\} \cup \mathbb{N} \mid 0 \leq n \leq n_{1}^{\lambda}\right\},} \\
& {\left[n_{i}^{\lambda}\right]=\left\{n \in \mathbb{N} \mid n_{i-1}^{\lambda}<n \leq n_{i}^{\lambda}\right\}, i \in\{2, \ldots, k\},} \\
& {\left[n_{k+1}^{\lambda}\right]=\left\{n \in \mathbb{N} \mid n_{k}^{\lambda}<n\right\} .}
\end{aligned}
$$

This implies that the terms of the form

$$
N_{\lambda(k)}=\{\{0\},\{1\}, \ldots,\{k-1\},[k]\}, k \in \mathbb{N},
$$

determine a cofinal sequence in $(\boldsymbol{N}, \leq)_{\underline{0}}$. Similarly, each term $\left(W_{\mu}, \leq^{\prime}\right), \mu \in$ $M_{0}$, having $\left|W_{\mu}\right| \geq 2$, is an ordered set of the form

$$
W_{\mu}=\left\{\left[n_{1}^{\mu}\right], \ldots,\left[n_{k+1}^{\mu}\right]\right\}, k \in \mathbb{N}, n_{i}^{\mu} \in\{0\} \cup \mathbb{N}, i \in\{1, \ldots, k+1\},
$$

where

$$
\begin{aligned}
& {\left[n_{1}^{\mu}\right]=\left\{ \pm n \in\{0\} \cup( \pm \mathbb{N}) \mid 0 \leq n \leq n_{1}^{\mu} \wedge 0 \geq-n \geq-n_{1}^{\mu}\right\}} \\
& {\left[n_{i}^{\mu}\right]=\left\{ \pm n \in \pm \mathbb{N} \mid n_{i-1}^{\mu}<n \leq n_{i}^{\mu} \wedge-n_{i-1}^{\mu}>-n \geq-n_{i}^{\mu}\right\}, i \in\{2, \ldots, k\}} \\
& {\left[n_{k+1}^{\mu}\right]=\left\{ \pm n \in \pm \mathbb{N} \mid n_{k}^{\mu}<n \wedge-n>-n_{k}^{\mu}\right\}}
\end{aligned}
$$

This implies that the terms of the form

$$
W_{\mu(k)}=\{\{0\},\{-1,1\}, \ldots,\{-k+1, k-1\},[k]\}, k \in \mathbb{N},
$$

determine a cofinal sequence in $\left(\boldsymbol{W}, \leq^{\prime}\right)_{\underline{0}}$. Notice that both $\left(N_{\lambda(k)}, \leq\right)$ and $\left(W_{\mu(k)}, \leq^{\prime}\right)$ are isomorphic to the segment $(\{1,2, \ldots, k+1\}, \leq)$ of $(\mathbb{N}, \leq)$. Then the inclusion $j:(\{0\} \cup \mathbb{N}, \leq) \hookrightarrow\left(\mathbb{Z}, \leq^{\prime}\right)$ induces in an obvious way the isomorphism of the corresponding cofinal sequences in $(\boldsymbol{N}, \leq)_{\underline{0}}$ and $\left(\boldsymbol{W}, \leq^{\prime}\right)_{\underline{0}}$. The conclusion follows.

Let us now prove that $(\boldsymbol{N}, \leq)_{\underline{0}}$ and $(\boldsymbol{Z}, \leq)_{\underline{0}}$ are isomorphic objects of pro-Ordg $g_{\underline{0}}$, where $(\boldsymbol{Z}, \leq)_{\underline{0}}$ belongs to the canonical $\operatorname{Ord} g_{\underline{0}}$-expansion

$$
\boldsymbol{r}_{\underline{0}}=\left(r_{\gamma}\right):(\mathbb{Z}, \leq) \rightarrow(\boldsymbol{Z}, \leq)_{\underline{0}}=\left(\left(Z_{\gamma}, \leq\right), r_{\gamma \gamma^{\prime}}, \Gamma_{\underline{0}}\right)
$$

of $(\mathbb{Z}, \leq)$. The terms $\left(N_{\lambda}, \leq\right)$ of $(\boldsymbol{N}, \leq)_{0}$, having $\left|N_{\lambda}\right| \geq 2$, are already described. A term $\left(Z_{\gamma}, \leq\right)$ of $(\boldsymbol{Z}, \leq)_{\underline{0}}$, having $\left|Z_{\gamma}\right| \geq 2$, is an ordered set of the form

$$
Z_{\gamma}=\left\{\left[m_{1}^{\gamma}\right], \ldots,\left[m_{k+1}^{\gamma}\right]\right\}, k \in \mathbb{N}, m_{i}^{\gamma} \in \mathbb{Z}, i \in\{1, \ldots, k+1\}
$$

where

$$
\begin{aligned}
& {\left[m_{1}^{\gamma}\right]=\left\{m \in \mathbb{Z} \mid m \leq m_{1}^{\gamma}\right\},} \\
& {\left[m_{i}^{\mu}\right]=\left\{m \in \mathbb{Z} \mid m_{i-1}^{\mu}<m \leq m_{i}^{\mu}\right\}, i \in\{2, \ldots, k\},} \\
& {\left[m_{k+1}^{\gamma}\right]=\left\{m \in \mathbb{Z} \mid m_{k}^{\gamma}<m\right\} .}
\end{aligned}
$$


Thus, the terms $Z_{\gamma(k)}, k \in \mathbb{N}$, of the form $\left(Z_{\gamma(1)}=\{[0]=\{\mathbb{Z}\}\}\right)$

$$
Z_{\gamma(2 n)}=\{[-n+1],\{-n+2\}, \ldots,\{0\}, \ldots,\{n-1\},[n]\},
$$

and

$$
Z_{\gamma(2 n+1)}=\{[-n],\{-n+1\}, \ldots,\{0\}, \ldots,\{n-1\},[n]\}
$$

determine a cofinal sequence in $(\boldsymbol{Z}, \leq)_{0}$ : Since both $\left(N_{\lambda(k)}, \leq\right)$ and $\left(Z_{\gamma(k)}, \leq\right)$ are isomorphic to the segment $(\{1,2, \ldots, k+1\}, \leq)$ of $(\mathbb{N}, \leq)$, the conclusion follows as in the previous case.

Observe that Example 3 generalizes from $\left(\mathbb{Z}, \leq^{\prime}\right)$ (Example 1 - diagram) to any " 0 -wedge of $(\{0\} \cup \mathbb{N})$-rays" ordered by "to be on the right side of". The next example is even more interesting.

ExAmple 5.7. Let $(\mathbb{Q}, \leq)$ and $(\mathbb{R}, \leq)$ be the sets of rational and real numbers, respectively, with the standard orderings. We will prove that $(\mathbb{Q}, \leq)$, $(\mathbb{R} \backslash \mathbb{Q}, \leq)$ and $(\mathbb{R}, \leq)$ have the same finite shape, i.e., that $S h_{\underline{0}}(\mathbb{Q}, \leq)=$ $S h_{\underline{0}}(\mathbb{R} \backslash \mathbb{Q}, \leq)=S h_{\underline{0}}(\mathbb{R}, \leq)$. Let

$$
\boldsymbol{p}_{\underline{0}}=\left(p_{\lambda}\right):(\mathbb{Q}, \leq) \rightarrow\left(\left(Q_{\lambda}, \leq\right), p_{\lambda \lambda^{\prime}}, \Lambda_{\underline{0}}\right)
$$

and

$$
\boldsymbol{q}_{\underline{0}}=\left(q_{\mu}\right):(\mathbb{R}, \leq) \rightarrow\left(\left(R_{\mu}, \leq\right), q_{\mu \mu^{\prime}}, M_{\underline{0}}\right)
$$

be the canonical $\operatorname{Or} d g_{0}$-expansions of $(\mathbb{Q}, \leq)$ and $(\mathbb{R}, \leq)$ respectively. Consider a term $Q_{\lambda}$ having $\left|Q_{\lambda}\right|=k+1 \geq 2$. The congruence classes $I_{1}, I_{2}, \ldots, I_{k+1}$ (elements of $\left.Q_{\lambda}\right)$ are disjoint intervals in $(\mathbb{Q}, \leq)$ such that

- $I_{1}$ is of the form $\left\langle\cdot, x_{1}\right]=\left\{q \in \mathbb{Q} \mid q \leq x_{1}\right\}, x_{1} \in \mathbb{Q}$, or $\left\langle\cdot, x_{1}\right\rangle=\{q \in$ $\left.\mathbb{Q} \mid q<x_{1}\right\}, x_{1} \in \mathbb{R}$

- $I_{i}$ is of the form $\left[x_{i-1}, x_{i}\right], x_{i-1} \leq x_{i}$ in $\mathbb{Q}$, or $\left\langle x_{i-1}, x_{i}\right], x_{i-1} \in \mathbb{R}$, $x_{i} \in \mathbb{Q}$, or $\left[x_{i-1}, x_{i}\right\rangle, \mathrm{x}_{i-1} \in \mathbb{Q}, x_{i} \in \mathbb{R}$, or $\left\langle x_{i-1}, x_{i}\right\rangle, \mathrm{x}_{i-1}<x_{i}$ in $\mathbb{R}$ ( $\mathrm{I}_{i}$ partially depends on $\left.I_{i-1}\right), i \in\{2, \ldots, k\}$;

- $I_{k+1}$ is of the form $\left[x_{k}, \cdot\right\rangle=\left\{q \in \mathbb{Q} \mid x_{k} \leq q\right\}, x_{k} \in \mathbb{Q}$, or $\left\langle x_{k}, \cdot\right\rangle=\{q \in$ $\left.\mathbb{Q} \mid x_{k}<q\right\}, \mathrm{x}_{k} \in \mathbb{R}\left(\mathrm{I}_{k+1}\right.$ is determined by $\left.I_{k}\right)$, and

- $I_{1}<I_{2}<\cdots<I_{k+1}$.

The finest ones $Q_{\lambda}$ 's alternate open intervals and singletons having $I_{1}$ and $I_{k+1}$ to be the proper intervals, while there is no singleton whenever the "meeting point" of two intervals is irrational. Notice that such a $\left(Q_{\lambda}, \leq\right)$ is isomorphic to the segment $(\{1,2, \ldots, k+1\}, \leq)$ of $(\mathbb{N}, \leq)$. Since a "meeting point" $\left(x_{i}-\right.$ the "end" of $I_{i}$ ) may belong to $\mathbb{R} \backslash \mathbb{Q}$ as well (a cut in $(\mathbb{Q}, \leq)$ ), the cardinality of $\Lambda_{0}$ equals $|\mathbb{R}|=c$. To be more precise, an $x_{i}$ can be a $q_{i} \equiv q_{i}^{ \pm}$- if it is rational, or an $r_{i} \equiv r_{i}^{-}$- if it is irrational, such that $q_{i}^{-}$means that $q_{i} \notin I_{i}$, while $q_{i}^{+}$means that $q_{i} \in I_{i}$. (For instance, if $k=4$ and $\left(x_{1}, x_{2}, x_{3}, x_{4}\right)=$ $\left(r_{1}^{-}, q_{2}^{-}, q_{3}^{+}, r_{4}^{-}\right)$, it means that $r_{1}<q_{2} \leq q_{3}<r_{4} . ; q_{2} \notin I_{2}, q_{3} \in I_{3} ;$ if $q_{3}=q_{2}$, then $I_{3}=\left\{x_{3}\right\}=\left\{q_{2}\right\}$.) Therefore, we may identify every such $\left(Q_{\lambda}, \leq\right)$, i.e., every $\lambda \in \Lambda_{\underline{0}}$, with a unique increasing $k$-tuple $\left(x_{1}, x_{2}, \ldots, x_{k}\right)$, where 
each $x_{i}$ is some $q_{i}^{ \pm}$or $r_{i}^{-}$, that yields a (canonical) bijection of the set of all such $k$-tuples $\left(x_{1}, x_{2}, \ldots, x_{k}\right), k \in \mathbb{N}$, onto $\Lambda_{0}$. (Recall that, by the general construction, in the case of $\left|Q_{\lambda}\right|=k+1=\left|Q_{\lambda^{\prime}}\right|, \lambda \leq \lambda^{\prime} \Leftrightarrow \lambda=\lambda^{\prime}$.)

In the same way, each term $R_{\mu}$ having $\left|R_{\mu}\right|=k+1 \geq 2$, consists of the congruence classes $I_{1}^{\prime}, I_{2}^{\prime}, \ldots, I_{k+1}^{\prime}$ (elements of $R_{\mu}$ ) that are disjoint intervals in $(\mathbb{R}, \leq)$ such that

- $I_{1}^{\prime}$ is of the form $\left\langle\cdot, x_{1}^{\prime}\right]$ or $\left\langle\cdot, x_{1}^{\prime}\right\rangle, x_{1}^{\prime} \in \mathbb{R}$;

- $I_{i}^{\prime}$ is of the form $\left[x_{i-1}^{\prime}, x_{i}^{\prime}\right], x_{i-1}^{\prime} \leq x_{i}^{\prime}$ in $\mathbb{R}$, or $\left\langle x_{i-1}^{\prime}, x_{i}^{\prime}\right]$, or $\left[x_{i-1}^{\prime}, x_{i}^{\prime}\right\rangle$, or $\left\langle x_{i-1}^{\prime}, x_{i}^{\prime}\right\rangle, x_{i-1}^{\prime}<x_{i}^{\prime}$ in $\mathbb{R}\left(I_{i}^{\prime}\right.$ partially depends on $\left.I_{i-1}^{\prime}\right), i \in$ $\{2, \ldots, k\}$

- $I_{k+1}^{\prime}$ is of the form $\left[x_{k}^{\prime}, \cdot\right\rangle$ or $\left\langle x_{k}^{\prime}, \cdot\right\rangle x_{k}^{\prime} \in \mathbb{R}\left(I_{k+1}^{\prime}\right.$ is determined by $\left.I_{k}^{\prime}\right)$, and

- $I_{1}^{\prime}<I_{2}^{\prime}<\cdots<I_{k+1}^{\prime}$.

The finest ones $R_{\mu}$ 's alternate open intervals and singletons having $I_{1}$ and $I_{k+1}$ to be the proper intervals. Clearly, the cardinality of $M_{\underline{0}}$ equals $|\mathbb{R}|=c$, and each $\left(R_{\mu}, \leq\right)$ is isomorphic to each $\left(Q_{\lambda}, \leq\right)$.of the same cardinality. As before, we may identify every such $\left(R_{\mu}, \leq\right)$, i.e., every $\mu \in M_{\underline{0}}$, with a unique increasing $k$-tuple $\left(x_{1}^{\prime}, x_{2}^{\prime}, \ldots, x_{k}^{\prime}\right)$, where each $x_{i}^{\prime}$ is some $q_{i}^{ \pm}$or $r_{i}^{ \pm}$, and obtain a (canonical) bijection of the set of all such $k$-tuples $\left(x_{1}^{\prime}, x_{2}^{\prime}, \ldots, x_{k}^{\prime}\right), k \in \mathbb{N}$, onto $M_{\underline{0}}$. (Again, if $\left|R_{\mu}\right|=k+1=\left|R_{\mu^{\prime}}\right|$, then $\mu \leq \mu^{\prime} \Leftrightarrow \mu=\mu^{\prime}$.)

The above consideration makes possible to define a function

$$
\varphi: M_{\underline{0}} \rightarrow \Lambda_{\underline{0}}
$$

in the following way. Let $\mu \in M_{0}$. If $\left|R_{\mu}\right|=1\left(\sim_{\mu}=\mathbb{R} \times \mathbb{R}\right)$, we put $\varphi(\mu)=\lambda$ such that $\left|Q_{\lambda}\right|=1\left(\sim_{\lambda}=\mathbb{Q} \times \mathbb{Q}\right)$. Let $\left|R_{\mu}\right|=k+1$ and let $\left(x_{1}^{\prime}, x_{2}^{\prime}, \ldots, x_{k}^{\prime}\right)$ be canonically associated with $\mu$.

CASE 1. The term $\left(R_{\mu}, \leq\right)$, corresponding to $\left(x_{1}^{\prime}, x_{2}^{\prime}, \ldots, x_{k}^{\prime}\right)$, does not contain any irrational singleton. Then $\left(x_{1}, x_{2}, \ldots, x_{k}\right)$ such that, for every $i \in\{1, \ldots, k\}, x_{i}=x_{i}^{\prime}$, corresponds to a unique $\lambda \in \Lambda_{\underline{0}}$. Put $\varphi(\mu)=\lambda$. Notice that, since $x_{i}^{\prime}=r_{i}^{ \pm}$and $x_{i}=r_{i}$ is possible, every $\lambda \in \Lambda_{0}$ is a $\varphi(\mu)$, for some (at most finitely many) $\mu$ of the considered kind. Further, if $\mu \leq \mu^{\prime}$ then $\varphi(\mu) \leq \varphi\left(\mu^{\prime}\right)$.

CASE 2. The term $\left(R_{\mu}, \leq\right)$, corresponding to $\left(x_{1}^{\prime}, x_{2}^{\prime}, \ldots, x_{k}^{\prime}\right)$, contains some irrational singletons. Then there are at most $\left[\frac{k}{2}\right]$ such singletons $I_{i}^{\prime}=$ $\left\{x_{i}^{\prime}\right\}=\left\{r_{i}\right\}, r_{i} \in \mathbb{R} \backslash \mathbb{Q}$, because the neighbors $I_{i-1}^{\prime}$ and $I_{i+1}^{\prime}$ must be proper intervals. Thus, the proper interval $I_{i+1}^{\prime}$ can be divided into two subintervals, $I_{i+1,1}^{\prime}$ and $I_{i+1,2}^{\prime}, I_{i+1,1}^{\prime}<I_{i+1,2}^{\prime}$. Put $I_{i}=I_{i+1,1}^{\prime} \cap \mathbb{Q}$ and $I_{i+1}=I_{i+1,2}^{\prime} \cap \mathbb{Q}$. In this way, inductively, by increasing indices $i=2, \ldots, k$, is constructed an increasing $k$-tuple $\left(x_{1}=x_{1}^{\prime}, x_{2}, \ldots, x_{k}\right)$ that corresponds to a unique $\lambda \in \Lambda_{0}$, and $\left(Q_{\lambda}, \leq\right) \cong\left(R_{\mu}, \leq\right)$. Then put $\varphi(\mu)=\lambda$.

One readily sees that the constructed function $\varphi: M_{0} \rightarrow \Lambda_{0}$ is an increasing surjection (not a bijection!) 
Now, for every $\mu \in M_{\underline{0}}$, put

$$
f_{\mu}:\left(Q_{\varphi(\mu)}, \leq\right) \rightarrow\left(R_{\mu}, \leq\right)
$$

to be the unique isomorphism. The conclusion follows, i.e.,

$$
S h_{\underline{0}}(\mathbb{Q}, \leq)=S h_{\underline{0}}(\mathbb{R}, \leq) .
$$

In a quite similar manner one can prove that

$$
S h_{\underline{0}}(\mathbb{R} \backslash \mathbb{Q}, \leq)=S h_{\underline{0}}(\mathbb{R}, \leq) .
$$

Remark 5.8. (a) Observe that, if $\lambda$ and $\lambda^{\prime}$ are congruences on a poset $(X, \leq)$ such that $(X / \lambda, \leq)$ and $\left(X / \lambda^{\prime}, \leq\right)$ are totally (well-) ordered, then $\left(X /\left(\lambda \cap \lambda^{\prime}\right), \leq\right)$ is totally (well-) ordered. Therefore, it is possible and makes sense to consider the quotient shapes of partially (totally) ordered sets with respect to the totally or well-ordered (well-ordered) sets. In fact, Examples 3 and 4 are of such a kind.

(b) According to Example 4, the analogues of Example 3 for $(\mathbb{Q}, \leq)$, $(\mathbb{R} \backslash \mathbb{Q}, \leq)$ and $(\mathbb{R}, \leq)$ (in several additional variations) can be constructed. By this, the new ordering $\leq^{\prime}$ on $\mathbb{R}$, is defined by the following rules:

(1) $\left((\forall r \in \mathbb{R}) 0 \leq^{\prime} r\right) \wedge\left(\leq^{\prime}\right.$ on $\mathbb{R}^{+}$is $\left.\leq\right) \wedge\left(\leq^{\prime}\right.$ on $\mathbb{R}^{-}$is $\left.\geq\right)$;

(2) $\left(\forall r \in \mathbb{R}^{-}\left(\forall r^{\prime} \in \mathbb{R}^{+}\right)\left\{\begin{array}{l}r \leq^{\prime} r^{\prime} \Leftrightarrow|r|<r^{\prime} \\ r^{\prime} \leq^{\prime} r \Leftrightarrow r^{\prime}<|r|\end{array}\right.\right.$

(the only unrelated pairs are $\{-r, r\}, r \in \mathbb{R} !$ ).

EXAMPLE 5.9. By this example we shall prove that the ordered sets $(\mathbb{R}, \leq)$ and $(\mathbb{R} \backslash \mathbb{Q}, \leq)$ have the same $\aleph_{0}$-shape. However, they are not $\aleph_{0}$-stable (isomorphic to a countable ordered set), and thus, their $\aleph_{0}$-shape type differs from $(\mathbb{Q}, \leq)$. Let

$$
\begin{aligned}
& \boldsymbol{p}_{\aleph_{0}}=\left(p_{\lambda}\right):(\mathbb{R} \backslash \mathbb{Q}, \leq) \rightarrow(\boldsymbol{S}, \leq)_{\aleph_{0}}=\left(\left(S_{\lambda}, \leq\right), p_{\lambda \lambda^{\prime}}, \Lambda_{\aleph_{0}}\right), \\
& \boldsymbol{q}_{\aleph_{0}}=\left(q_{\mu}\right):(\mathbb{R}, \leq) \rightarrow(\boldsymbol{R}, \leq)_{\aleph_{0}}=\left(\left(R_{\mu}, \leq\right), q_{\mu \mu^{\prime}}, M_{\aleph_{0}}\right)
\end{aligned}
$$

be the appropriate canonical expansions. One may assume, without loss of generality, that all the sets $S_{\lambda}$ and $R_{\mu}$ are infinite. A typical (enough fine) $\left(R_{\mu}, \leq\right)$ consists of an increasing infinite countable alternating chain $\left(I_{j}\right)_{j \in J(\mu)}$ (not necessarily discrete and having nor min neither max) of open intervals and singletons (of $\mathbb{R}$ ) $I_{j} \equiv r_{j} \in R_{\lambda}$. A typical (enough fine) $S_{\lambda}$ one obtains of an (enough fine) $R_{\mu}$ by removing all of its rational singletons. This yields a unique surjection of $M_{\aleph_{0}}$ onto $\Lambda_{\aleph_{0}}$. Then the inclusion of $(\mathbb{R} \backslash \mathbb{Q}, \leq)$ into $(\mathbb{R}, \leq)$ induces an isomorphism of the corresponding canonical $\aleph_{0}$-expansions. Namely, there exists cofinally many isomorphisms $i_{\mu}:\left(S_{i(\mu)}, \leq\right) \rightarrow\left(R_{\mu}, \leq\right)$. To see that $(\mathbb{R}, \leq)$ is not $\aleph_{0}$-stable, let us assume to the contrary. Then there exist a countable ordered set $(X, \leq)$ and an $\aleph_{0}$-shape isomorphism $F \in$ $S h_{\aleph_{0}}((X, \leq),(\mathbb{R}, \leq))$. Thus, further, there exists an isomorphism

$$
\boldsymbol{f}=\left(f_{\mu}\right):\lfloor(X, \leq)\rfloor \rightarrow(\boldsymbol{R}, \leq)_{\aleph_{0}}, \quad q_{\mu \mu^{\prime}} f_{\mu^{\prime}}=f_{\mu},
$$


of pro-Ordg $g_{\aleph_{0}}$, having the inverse

$$
\boldsymbol{g}=\left\lfloor g^{\mu_{0}}\right\rfloor:(\boldsymbol{R}, \leq)_{\aleph_{0}} \rightarrow\lfloor(X, \leq)\rfloor, \quad g^{\mu_{0}}:\left(R_{\mu_{0}}, \leq\right) \rightarrow(X, \leq),
$$

for some $\mu_{0} \in M_{\aleph_{0}}$. Since all the $q_{\mu \mu^{\prime}}$ are (effective) epimorphisms, one readily sees that $f_{\mu_{0}}:(X, \leq) \rightarrow\left(R_{\mu_{0}}, \leq\right)$ is an isomorphism of $\operatorname{Ordg}$ and $f_{\mu_{0}}^{-1}=g^{\mu_{0}}$. However, if $\mu>\mu_{0}$, then $f_{\mu}$ cannot be an isomorphism. Indeed, if such an $f_{\mu}$ is an isomorphism, then $q_{\mu_{0} \mu}$ is an isomorphisms, that contradicts the general construction.

We finish this section with the following very interesting examples.

ExAMPLE 5.10. (a) Let $\left(\mathbb{N}, \leq^{*}\right)$ be the poset of Example $1(\mathrm{a})$, where $n \leq^{*} n^{\prime}$ means that $n$ factorizes (divides) $n^{\prime}\left(n^{\prime}=m n\right.$, for some $\left.m \in \mathbb{N}\right)$. Let us determine its canonical $\operatorname{Ord} g_{\underline{0}}$-expansion

$$
\boldsymbol{p}_{\underline{0}}=\left(p_{\lambda}\right):\left(\mathbb{N}, \leq^{*}\right) \rightarrow\left(\boldsymbol{N}, \leq^{*}\right)_{\underline{0}}=\left(\left(N_{\lambda}, \leq^{*}\right), p_{\lambda \lambda^{\prime}}, \Lambda_{\underline{0}}\right) .
$$

Let $\lambda \equiv \sim$ be a congruence on $\left(\mathbb{N}, \leq^{*}\right)$, and let $p:\left(\mathbb{N}, \leq^{*}\right) \rightarrow\left(\mathbb{N} / \lambda, \leq^{*}\right)$ be the (genuine order-preserving) quotient function. Since, for every $m, n \in \mathbb{N}$, $m \leq^{*} n m$ and $n \leq^{*} m n=n m$ hold, it follows that $[m] \leq^{*}[m n]$ and $[n] \leq^{*}$ $[n m]$ hold as well. Assume that $\lambda$ yields the quotient poset $\left(\mathbb{N} / \lambda, \leq^{*}\right)$ which is finite and not trivial, i.e., $|\mathbb{N} / \lambda|=k \in \mathbb{N}, k \geq 2$. Let $\left[n_{1}\right], \ldots,\left[n_{k}\right]$ be all the congruence classes. Then we may assume that, for every $i \in\{1, \ldots, k\}$, $n_{i}$ is the minimal (in the natural ordering) representative of $\left[n_{i}\right]$ and that $n_{1}=1<n_{2}<\cdots<n_{k}$ (in the natural ordering of $\mathbb{N}$ ). Further,

$$
(\forall i \in\{2, \ldots, k\}) \quad[1]<^{*}\left[n_{i}\right] .
$$

Thus, since at least one of $\left[n_{2}\right], \ldots,\left[n_{3}\right]$ is an infinite subset of $\mathbb{N}$, the class [1] must be the singleton $\{1\}$, and hence, $n_{2}=2$. Let us, firstly, consider the simplest case of $2 \leq k \leq 3$. If $k=2$, then, clearly, $n_{1}=1, n_{2}=2$ and

$$
[1]=\{1\}<^{*}[2]=\mathbb{N} \backslash\{1\}
$$

must hold. If $k=3$, then or $[2]<^{*}\left[n_{3}\right]$ either [2] $\nless^{*}\left[n_{3}\right]$. In the first subcase, [2] must be a finite subset of $\mathbb{N}$, while $\left[n_{3}\right]$ must contain all $n \in \mathbb{N}$ such that $n \geq \max [2]+1$. However, then

$$
[1]=\{1\}<^{*}[2]<^{*}\left[n_{3}\right] \supseteq[\max [2]+1]
$$

must hold - a contradiction (because there exists a prime number $p>2$ in the class $\left.\left[n_{3}\right]\right)$. Thus, the second subcase, i.e., $[2] \nless^{*}\left[n_{3}\right]$ must occur. Since $\left[n_{3}\right] \nless^{*}[2]$ as well, and since the quotient function is order-preserving,

$$
(\forall n \in[2])\left(\forall n^{\prime} \in\left[n_{3}\right) \quad\left(n \nless^{*} n^{\prime} \wedge n^{\prime} \nless n\right) .\right.
$$

must hold. However, one readily sees that such a partition $\left\{[2],\left[n_{3}\right]\right\}$ of $\mathbb{N} \backslash\{1\}$ cannot exist. Consequently, the case of $2 \leq k \leq 3$ reduces to $k=2$. A quite similar analysis shows that the case $2 \leq k \leq 4$ reduces to $2 \leq k \leq 3$, and thus, to $k=2$. And in general, the case $2 \leq k \leq m+1, m \in \mathbb{N}$, reduces to 
$2 \leq k \leq m$. Therefore, by induction, the only non-trivial possibility is $k=2$. Therefore, the canonical $\operatorname{Ord} g_{0}$-expansion $\boldsymbol{p}_{0}$ of $\left(\mathbb{N}, \leq^{*}\right)$ is

$$
\left(\{\mathbb{N}\}, \leq^{*}\right) \stackrel{p_{12}}{\leftarrow}\left(\{\{1\}, \mathbb{N} \backslash\{1\}\}, \leq^{*}\right) \stackrel{p_{2}}{\leftarrow}\left(\mathbb{N}, \leq^{*}\right),
$$

that is isomorphic (in pro-Ordgo ${ }_{0}$ ) to

$$
\lfloor p\rfloor:\left(\mathbb{N}, \leq^{*}\right) \rightarrow\left(\{\{1\}, \mathbb{N} \backslash\{1\}\}, \leq^{*}\right) .
$$

Consequently,

$$
\boldsymbol{p}_{0}^{\prime}=\left\lfloor p^{\prime}\right\rfloor:\left(\mathbb{N}, \leq^{*}\right) \rightarrow(\{1,2\}, \leq), p^{\prime}(n)=\left\{\begin{array}{l}
1, n=1 \\
2, n \in \mathbb{N} \backslash\{1\}
\end{array},\right.
$$

is a rudimentary $\operatorname{Ord} g_{0}$-expansion of $\left(\mathbb{N}, \leq^{*}\right)$. Therefore, the poset $\left(\mathbb{N}, \leq^{*}\right)$ is (finitely) stable ( $\underline{0}-$ stable), i.e., it is isomorphic to an object of $\operatorname{Ord} g_{0}$. (Observe that the canonical $\aleph_{0}$-expansion, as well as every $\kappa^{-}$- and $\kappa$-expansion of $\left(\mathbb{N}, \leq^{*}\right),, \kappa>\aleph_{0}$, is the identity morphism.)

(b) Let us extend $\left(\mathbb{N}, \leq^{*}\right)$ to $\left(\mathbb{N}_{\infty}, \leq^{*}\right)$ by adding a unique maximal element $\infty$ such that, for every $n \in \mathbb{N}_{\infty}=\mathbb{N} \cup\{\infty\}, n \leq^{*} \infty$. Then, quite similarly to the case (a) (via the canonical $\operatorname{Or} d g_{0}$-expansion $\boldsymbol{q}_{0}$ ), one obtains a rudimentary $\operatorname{Ordg} g_{\underline{0}}-$ expansion

$$
\boldsymbol{q}_{\underline{0}}^{\prime}=\left\lfloor q^{\prime}\right\rfloor:\left(\mathbb{N}_{\infty}, \leq^{*}\right) \rightarrow(\{1,2,3\}, \leq), q^{\prime}(n)=\left\{\begin{array}{l}
1, n=1 \\
2, n \in \mathbb{N} \backslash\{1\} . \\
3, n \equiv \infty
\end{array} .\right.
$$

Thus, the posets $\left(\mathbb{N}, \leq^{*}\right)$ and $\left(\mathbb{N}_{\infty}, \leq^{*}\right)$ are both (finitely) stable having different finite shapes.

\section{The Shapes of PSEUdometric SPACES}

6.1. The general case. An analysis shows that the (general) quotient shape theory (by means of infinite cardinals) applied to pseudometric (gauge; [5], Ch. IX, Sec. 10) spaces (endowed with various kinds of convenient classes of morphisms) has some obstacles. First of all, one has to define a certain category of pseudometric spaces, i.e., a suitable class of functions should be chosen to become the needed class of morphisms. Although a pseudometric on a set induces a topology (having as a basis the family of all pseudoballs), it is not often usual to consider pseudometric spaces as the topological (pseudometrizable) spaces. Still, let us denote by $M e t \subseteq P M e t$ the corresponding categories of all pseudo(metric) spaces and their continuous functions. We will abandon, for a moment, the case with continuous functions by attempting to find a larger class of suitable morphisms of pseudometric spaces.

Let $(X, \rho)$ and $(Y, \sigma)$ be pseudometric spaces and let $f: X \rightarrow Y$ be a function. Then $f$ is said to be admissible (with respect to $\rho$ and $\sigma$ ) if the following condition is fulfilled:

$$
\left(\forall x_{1}, x_{2} \in X\right) \quad \rho\left(x_{1}, x_{2}\right)=0 \Rightarrow \sigma\left(f\left(x_{1}\right), f\left(x_{2}\right)\right)=0 .
$$


Notice that, if $\rho$ is a metric, then every function $f: X \rightarrow Y$ is admissible (for $\rho$ and any $\sigma$ ). There obviously exists the corresponding category $P M e t^{a}$ of all pseudometric spaces and their admissible functions. Then the category $\mathrm{Met}^{a}$ of all metric spaces and functions is a full subcategory of $P M t^{a}$. (Some other possibilities are given in Remark 5 , (a) and (b), below.) In order to get a pseudometric quotient space $\left(X_{\lambda}, \rho_{\lambda}\right)$ on $X_{\lambda} \equiv X / \lambda$, the equivalence relation $\sim \equiv \lambda$ on $X$ should fulfill the following condition: For every pair $x, x^{\prime} \in X$,

$$
x \sim x^{\prime} \Rightarrow \rho_{\lambda}\left([x]_{\lambda},\left[x^{\prime}\right]_{\lambda}\right)=\rho_{\lambda}\left([x]_{\lambda},[x]_{\lambda}\right)=0 .
$$

Further, since the quotient function $p_{\lambda}: X \rightarrow X_{\lambda}, p_{\lambda}(x)=[x]_{\lambda}$, should be admissible with respect to $\rho$ and $\rho_{\lambda}$,

$$
\rho\left(x, x^{\prime}\right)=0 \Rightarrow \rho_{\lambda}\left([x]_{\lambda},\left[x^{\prime}\right]_{\lambda}\right)=0
$$

must hold. Therefore, we define an equivalence relation $\lambda \equiv \sim$ on $X$ to be a congruence on $(X, \rho)$ provided the following condition is fulfilled:

$$
x \sim x^{\prime} \Rightarrow \rho\left(x, x^{\prime}\right)=0 .
$$

It is readily seen that a congruence $\lambda$ on $(X, \rho)$ admits a function $\rho_{\lambda}: X_{\lambda} \times$ $X_{\lambda} \rightarrow \mathbb{R}$ defined by

$$
\rho_{\lambda}\left(\left[x_{1}\right]_{\lambda},\left[x_{2}\right]_{\lambda}\right)=\rho\left(x_{1}, x_{2}\right),
$$

Namely, if $x_{1}, x_{1}^{\prime}, x_{2}, x_{2}^{\prime} \in X$ such that $\left[x_{1}\right]=\left[x_{1}^{\prime}\right]$ and $\left[x_{2}\right]=\left[x_{2}^{\prime}\right]$, then the triangle inequality yields $\rho\left(x_{1}, x_{2}\right) \leq \rho\left(x_{1}^{\prime}, x_{2}^{\prime}\right)$ and $\rho\left(x_{1}^{\prime}, x_{2}^{\prime}\right) \leq \rho\left(x_{1}, x_{2}\right)$, and thus $\rho\left(x_{1}, x_{2}\right)=\rho\left(x_{1}^{\prime}, x_{2}^{\prime}\right)$. Moreover, one easily verifies that $\rho_{\lambda}$ is a pseudometric on $X_{\lambda}$ and that the quotient function $p_{\lambda}:(X, \rho) \rightarrow\left(X_{\lambda}, \rho_{\lambda}\right)$ is admissible.

Observe that every congruence on $(X, \rho)$ divides each subset

$$
R_{x}=\left\{x^{\prime} \mid \rho\left(x, x^{\prime}\right)=0\right\} \subseteq X \text { separately. }
$$

Thus, there exists the largest congruence $\sim_{\rho} \equiv \lambda_{\rho}$ on $(X, \rho)$, which is determined by

$$
x \sim_{\rho} x^{\prime} \Leftrightarrow \rho\left(x, x^{\prime}\right)=0 .
$$

Clearly, for every congruence $\lambda,\left|X_{\lambda \rho}\right| \leq\left|X_{\lambda}\right|$.

If we now want to proceed in the general way of constructing the quotient $\kappa^{-}$-shape ( $\kappa$-shape) categories, we have to be aware of the following obstacle: If $\left|X_{\lambda_{\rho}}\right| \geq \kappa_{0}\left(\left|X_{\lambda_{\rho}}\right|>\kappa_{0}\right)$, then, for every infinite $\kappa \leq \kappa_{0}\left(\kappa<\kappa_{0}\right)$, there does not exist the canonical $\boldsymbol{p}_{\kappa^{-}}$-expansion $\left(p_{\kappa^{-}}\right.$expansion) of $(X, \rho)$. One can overcome this obstacle by passing to the appropriate subcategories. (Consider the full subcategories $P M e t_{\kappa_{*}}^{a}, P M e t_{\kappa^{*}}^{a} \subseteq P M e t^{a}$ determined by all the objects $(X, \rho)$ such that $\left|X_{\lambda_{\rho}}\right|<\kappa,\left|X_{\lambda_{\rho}}\right| \leq \kappa$, respectively; see the next subsection.) However, the factorization condition (E1) cannot be successfully solved (compare Lemma 7 below and its proof). Therefore, we shall consider the largest congruence on $(X, \rho)$ only. 
Denote $X_{\rho}=X_{\lambda_{\rho}} \equiv X / \lambda_{\rho}$ the (smallest) quotient set, and denote the pseudometric $\rho_{\lambda_{\rho}}$ by

$$
d_{\rho}: X_{\rho} \times X_{\rho} \rightarrow \mathbb{R}, d_{\rho}\left(\left[x_{1}\right],\left[x_{2}\right]\right)=\rho\left(x_{1}, x_{2}\right) .
$$

Lemma 6.1. For every pseudometric space $(X, \rho)$, the quotient $\left(X_{\rho}, d_{\rho}\right)$ is a metric space and $p:(X, \rho) \rightarrow\left(X_{\rho}, d_{\rho}\right), p(x)=[x]$, is an admissible function. Moreover, $d_{\rho}$ is an ultrametric if and only if, $\rho$ is an ultrapseudometric.

Proof. Notice that $d_{\rho}$ inherits all the pseudometric properties of $\rho$. Further,

$$
d_{\rho}\left(\left[x_{1}\right],\left[x_{2}\right]\right)=\rho\left(x_{1}, x_{2}\right)=0 \Rightarrow x_{1} \sim_{\rho} x_{2} \Rightarrow\left[x_{1}\right]=\left[x_{2}\right] .
$$

Thus, $d_{\rho}$ is a metric. The quotient function $p$ is admissible because $\sim_{\rho}$ is a congruence. If $\rho$ is an ultrapseudometric, i.e., the following strengthening of the triangle inequality

$$
\left(\forall x, x^{\prime}, x^{\prime \prime} \in X\right) \quad \rho\left(x, x^{\prime}\right) \leq \max \left\{\rho\left(x, x^{\prime \prime}\right), \rho\left(x^{\prime \prime}, x^{\prime}\right)\right\},
$$

holds, then the same holds for $d_{\rho}$. Conversely, if $d_{\rho}$ is an ultrametric, then, for all $x, x^{\prime}, x^{\prime \prime} \in X$,

$$
\begin{aligned}
\rho\left(x, x^{\prime}\right) & \left.=d_{\rho}\left([x],\left[x^{\prime}\right]\right) \leq \max \left\{d_{\rho}\left([x],\left[x^{\prime \prime}\right]\right), d_{\rho}\left(f x^{\prime \prime}\right],\left[x^{\prime}\right]\right)\right\} \\
& =\max \left\{\rho\left(x, x^{\prime \prime}\right), \rho\left(x^{\prime \prime}, x^{\prime}\right)\right\} .
\end{aligned}
$$

Hence, $\rho$ is an ultrapseudometric.

To go further, let us observe that an $f:(X, \rho) \rightarrow(Y, \sigma)$ is admissible (i.e., $\left.\rho\left(x, x^{\prime}\right)=0 \Rightarrow \sigma\left(f(x), f\left(x^{\prime}\right)\right)=0\right)$ if and only if,

$$
\left(\forall x, x^{\prime} \in X\right) \quad x \sim_{\rho} x^{\prime} \Rightarrow f(x) \sim_{\sigma} f\left(x^{\prime}\right)
$$

(i.e., $f$ is $\left(\sim_{\rho}, \sim_{\sigma}\right)$-preserving.

Lemma 6.2. Let $f:(X, \rho) \rightarrow(Y, \sigma)$ be a morphism of PMet ${ }^{a}$. Then there exists a unique morphism $\tilde{f}:\left(X_{\rho}, d_{\rho}\right) \rightarrow\left(Y_{\sigma}, d_{\sigma}\right)$ of Met $^{a}$ such that $\tilde{f} p=q f$, i.e., the following diagram (in PMet ${ }^{a}$ ) commutes

$$
\begin{array}{cccc}
\left(X_{\rho}, d_{\rho}\right) & \stackrel{p}{\leftarrow} & (X, \rho) \\
\tilde{f} \downarrow & & \downarrow f \\
\left(Y_{\sigma}, d_{\sigma}\right) & \stackrel{q}{\leftarrow} & (Y, \sigma)
\end{array} .
$$

Proof. Since $f$ is admissible, it is $\left(\sim_{\rho}, \sim_{\sigma}\right)$-preserving. Thus, there exists a unique function

$$
\tilde{f}: X_{\rho} \rightarrow Y_{\sigma}, \tilde{f}([x])=[f(x)] .
$$

Hence, for every $x \in X$,

$$
\tilde{f} p(x)=\tilde{f}([x])=[f(x)]=q f(x) .
$$

Since $\left(X_{\rho}, d_{\rho}\right)$ is a metric space, the function $\tilde{f}:\left(X_{\rho}, d_{\rho}\right) \rightarrow\left(Y_{\sigma}, d_{\sigma}\right)$ belongs to $\operatorname{Mor}\left(M_{e t}{ }^{a}\right)$. 
The next lemma accomplishes Lemma 6,

LemMa 6.3. Let $f:(X, \rho) \rightarrow(Y, \delta)$ be a morphism of $\mathrm{PMet}^{a}$ with $(Y, \delta)$ a metric space. Then the equivalence relation $\sim_{f}$ on $X$, induced by fibers of $f$, is coarser than $\sim_{\rho}$, and there exists a unique morphism $\tilde{f}:\left(X_{\rho}, d_{\rho}\right) \rightarrow(Y, \delta)$ of Met $^{a}$ such that $\tilde{f} p=f$.

Proof. Since

$$
x \sim_{f} x^{\prime} \Leftrightarrow f(x)=f\left(x^{\prime}\right),
$$

and since $f$ is admissible and $\delta$ is a metric, it follows that

$$
x \sim_{\rho} x^{\prime} \Leftrightarrow \rho\left(x, x^{\prime}\right)=0 \Rightarrow \delta\left(f(x), f\left(x^{\prime}\right)\right)=0 \Leftrightarrow f(x)=f\left(x^{\prime}\right) \Leftrightarrow x \sim_{f} x^{\prime} .
$$

Thus, $\sim_{f}$ is coarser than $\sim_{\rho}$. Consequently, there exists a (set) function

$$
p^{\prime}: X_{\rho} \rightarrow X / \sim_{f}, \quad p^{\prime}([x])=[x]_{f} .
$$

Further, since $x \sim_{f} x^{\prime}$ implies $f(x)=f\left(x^{\prime}\right)$, there exists a function

$$
f^{\prime}: X / \sim_{f} \rightarrow Y, \quad f^{\prime}\left([x]_{f}\right)=f(x) .
$$

Put $\tilde{f}=f^{\prime} p^{\prime}: X_{\rho} \rightarrow Y$. Then

$$
\tilde{f}([x])=f^{\prime} p^{\prime}([x])=f^{\prime}\left([x]_{f}\right)=f(x) .
$$

Therefore, $\tilde{f} p=f$. Since $\left(X_{\rho}, d_{\rho}\right)$ is a metric space, the function $\tilde{f}$ is $\tilde{f}$ : $\left(X_{\rho}, d_{\rho}\right) \rightarrow(Y, \delta)$ belonging to $\operatorname{Mor}\left(M e t^{a}\right)$, and it is unique because $p$ is surjective.

The proven facts from above yield the following theorem and corollary.

ThEOREM 6.4. The category Met ${ }^{a}$ of all metric spaces and functions is a rudimentary pro-reflective subcategory of the category PMet ${ }^{a}$ of all pseudometric spaces and admissible functions. More precisely, for every pseudometric space $(X, \rho)$, the quotient (effective) epimorphism $p:(X, \rho) \rightarrow\left(X_{\rho}, d_{\rho}\right)$ is a rudimentary $M_{\text {et }}{ }^{a}$-expansion of $(X, \rho)$.

Corollary 6.5. For every $f:(X, \rho) \rightarrow(Y, \delta)$ of $P_{M e t}{ }^{a}$, with $(Y, \delta)$ a metric space, and every congruence $\lambda$ on $(X, \rho)$, there exists a unique factorization through $\left(X_{\lambda}, \rho_{\lambda}\right), f=f_{\lambda} p_{\lambda}$.

Proof. Observe that every congruence $\lambda$ is finer than $\sim_{\rho}$ and apply Theorem 4.

REMARK 6.6. (a) One can prove, in the same way, that Met $\subseteq$ PMet is also a rudimentary pro-reflective subcategory, i.e., every pseudometric space $(X, \rho)$ admits a rudimentary epimorphic Met-expansion $p:(X, \rho) \rightarrow\left(X_{\rho}, d_{\rho}\right)$ (all the functions are continuous).

(b) There are some other interesting possibilities of choosing suitable morphisms of pseudometric spaces. For instance, each of the two following ("equidistance preserving") properties of a function is categorical on pseudometric spaces: 
(1) $\rho\left(x_{1}, x_{2}\right)=\rho\left(x_{1}^{\prime}, x_{2}^{\prime}\right) \Rightarrow \sigma\left(f\left(x_{1}\right), f\left(x_{2}\right)\right)=\sigma\left(f\left(x_{1}^{\prime}\right), f\left(x_{2}^{\prime}\right)\right)$,

(2) $\sigma\left(f\left(x_{1}\right), f\left(x_{2}\right)\right)=\sigma\left(f\left(x_{1}^{\prime}\right), f\left(x_{2}^{\prime}\right)\right) \Rightarrow \rho\left(x_{1}, x_{2}\right)=\rho\left(x_{1}^{\prime}, x_{2}^{\prime}\right)$.

Notice that every function from a non null-pseudometric $\left(\rho \neq c_{0}\right)$ space to a null-pseudometric $\left(\sigma=c_{0}\right)$ space has property (1) but not (2), while the function $f:\left(\mathbb{R}, \rho=c_{0}\right) \rightarrow\left(\mathbb{R}^{2}, d_{2}\right), f(\xi)=(\xi, \xi)$, has property (2) but not (1). All $p_{\lambda}$ and $p_{\lambda \lambda^{\prime}}$, obtained by congruences, have both properties (1) and (2). Again, the appropriate metric categories (having property (1) or (2) or (1) and (2) simultaneously) are rudimentary pro-reflective subcategories of the corresponding pseudometric categories. Furthermore, property (2) admits the non-rudimentary shape theories for $P M e t_{\kappa^{-}}^{(2)} \subseteq P M e t_{\kappa_{*}}^{(2)}$ and $P M e t_{\kappa}^{(2)} \subseteq$ $P M e t_{\kappa^{*}}^{(2)}$. An analogue holds true in the special case of properties (1) and (2) simultaneously as well.

(c) It is well known fact ([13, I.2.1, Example 1]) that the topological category of realcompact spaces (compact Hausdorff spaces) is a rudimentary pro-reflective subcategory of the category of completely regular spaces - via the Hewitt realcompactification (the Stone-Cech compactification). However, except briefly in [17], Section 5, the corresponding shape theories were not studied.

ExAmple 6.7. Let $X=A \sqcup B$ (disjoint union), and let $\rho$ be a pseudometric on $X$ such that, for all $a, a^{\prime} \in A$ and all $b, b^{\prime} \in B$,

$$
\rho\left(a, a^{\prime}\right)=0=\rho\left(b, b^{\prime}\right), \quad \rho(a, b)>0 .
$$

Similarly, let $Y=C \sqcup D$, and let $\sigma$ be a pseudometric on $Y$ such that, for all $c, c^{\prime} \in C$ and all $d, d^{\prime} \in D$,

$$
\sigma\left(c, c^{\prime}\right)=0=\sigma\left(d, d^{\prime}\right), \quad \sigma(c, d)>0 .
$$

Then both quotient spaces $\left(X_{\rho}, d_{\rho}\right)$ and $\left(Y_{\sigma}, d_{\sigma}\right)$ are isomorphic (in Met $\left.^{a}\right)$ to the discrete (ultra)metric space $\{0,1\}$, and thus, $\operatorname{Sh}_{M e t^{a}}(X, \rho)=\operatorname{Sh}_{M e t^{a}}(Y, \sigma)$. However, if $|X| \neq|Y|$, then $(X, \rho) \not \approx(Y, \sigma)$ in PMet $^{a}$.

ExAmple 6.8. Let $X=\mathbb{R}^{2}$ and let $\rho: X \times X \rightarrow \mathbb{R}$ be defined by

$$
\rho\left(x, x^{\prime}\right)=\rho\left(\left(\xi^{1}, \xi^{2}\right),\left(\xi^{\prime 1}, \xi^{\prime 2}\right)\right)=\left|\xi^{1}-\xi^{\prime 1}\right| .
$$

Then $(X, \rho)$ is a pseudometric space. Let $Y=\mathbb{R}^{3}$ and let $\sigma: Y \times Y \rightarrow \mathbb{R}$ be defined by

$$
\sigma\left(y, y^{\prime}\right)=\sigma\left(\left(\eta^{1}, \eta^{2}, \eta^{3}\right),\left(\eta^{\prime 1}, \eta^{\prime 2}, \eta^{\prime 3}\right)\right)=\left|\eta^{1}-\eta^{\prime 1}\right|
$$

Then $(Y, \sigma)$ is a pseudometric space. Since $\left(X_{\rho}, d_{\rho}\right) \cong\left(Y_{\sigma}, d_{\sigma}\right) \cong \mathbb{R}$ (the Euclidean line) in $M e t$, it follows that

$$
S h_{M e t}(X, \rho)=S h_{M e t}(Y, \sigma) \quad\left(\text { and } S h_{M e t^{a}}(X, \rho)=\operatorname{Sh}_{M e t^{a}}(Y, \sigma)\right) .
$$

In both cases the shape representative may be $\mathbb{R}$. On the other side, one readily sees that $\left(\mathbb{R}^{2}, \rho\right) \cong\left(\mathbb{R}^{3}, \sigma\right)$ in $P$ Met ${ }^{a}$ as well (map each line $\{\xi\} \times \mathbb{R}$ 
bijectively to the corresponding plane $\{\xi\} \times \mathbb{R} \times \mathbb{R})$. However, in the continuous case, it is obvious that

$$
(X, \rho)=\left(\mathbb{R}^{2}, \rho\right) \not \approx\left(\mathbb{R}^{3}, \sigma\right)=(Y, \sigma)
$$

in PMet.

Example 6.9. Let $(X, \rho)$ and $Y$ be as in Example 8, and let the pseudometric $\sigma^{\prime}$ on $Y$ be defined by

$$
\sigma^{\prime}\left(y, y^{\prime}\right)=\sigma\left(\left(\eta^{1}, \eta^{2}, \eta^{3}\right),\left(\eta^{\prime 1}, \eta^{\prime 2}, \eta^{\prime 3}\right)\right)=\left(\left|\eta^{1}-\eta^{\prime 1}\right|^{2}+\left|\eta^{2}-\eta^{\prime 2}\right|^{2}\right)^{\frac{1}{2}} .
$$

Since $\left(X_{\rho}, d_{\rho}\right) \approx \mathbb{R}$ and $\left(Y_{\sigma^{\prime}}, d_{\sigma^{\prime}}\right) \approx \mathbb{R}^{2}$ (the Euclidean plane), the metric spaces $\left(X_{\rho}, d_{\rho}\right)$ and $\left(Y_{\sigma^{\prime}}, d_{\sigma^{\prime}}\right)$ are isomorphic in Met $^{a}$ (see Example 8). Therefore, $\operatorname{Sh}_{M e t^{a}}(X, \rho)=S h_{M e t^{a}}\left(Y, \sigma^{\prime}\right)$ and their (admissible) shape representative may be $\mathbb{R}$. However, in the continuous case, $S h_{M e t}(X, \rho)=S h_{\text {Met }}(\mathbb{R}) \neq$ $S h_{M e t}\left(\mathbb{R}^{2}\right)=S h_{M e t}\left(Y, \sigma^{\prime}\right)$.

6.2. The shapes of ultrapseudometric spaces. Recall again that an ultrapseudometric (ultrametric) on a set $X$ is a pseudometric (metric) $\rho: X \times X \rightarrow$ $\mathbb{R}$ satisfying the following strengthening of the triangle inequality:

$$
\left(\forall x, x^{\prime}, x^{\prime \prime} \in X\right) \quad \rho\left(x, x^{\prime}\right) \leq \max \left\{\rho\left(x, x^{\prime \prime}\right), \rho\left(x^{\prime \prime}, x^{\prime}\right)\right\} .
$$

An ultrapseudometric (ultrametric) space is a pseudometric (metric) space $(X, \rho)$ where $\rho$ is an ultrapseudometric (ultrametric). Let us firstly observe that a congruence $\sim \equiv \lambda$ on an ultrapseudometric space $(X, \rho)$, i.e.,

$$
x \sim x^{\prime} \Rightarrow \rho\left(x, x^{\prime}\right)=0 .
$$

induces an ultrapseudometric $\rho_{\lambda}$ on $X / \lambda \equiv X_{\lambda}$ by defining

$$
\rho_{\lambda}\left(\left[x_{1}\right]_{\lambda},\left[x_{2}\right]_{\lambda}\right)=\rho\left(x_{1}, x_{2}\right) .
$$

Therefore, everything we have established in the first subsection concerning Met $^{a} \subseteq$ PMet $^{a}$ (and the other cases mentioned in Remark 5, (a) and (b) ) remains valid for their full subcategories $U M e t^{a} \subseteq U P M e t^{a}$ of ultrapseudometric spaces and admissible (and the other mentioned classes of) functions.

Nevertheless, there is another interesting operable possibility in constructing ultrametric expansions of ultrapseudometric spaces, influenced by results obtained in [16]. By Theorem 4 of [16], there is a certain duality between ultrapseudometrics on a set $X$ and monotone families of equivalence relations on $X$. For the sake of simplicity, we will consider the subcategory of UPMet determined by $\operatorname{diam}(X, \rho) \leq 1$ (see [16], Lemma 1, and [16], Remark 5 (a)). By Theorem 1 of [16], every such ultrapseudometric $\rho$ on $X$ induces a unique decreasing (in general, not strictly decreasing) family of equivalence relations $\left(\sim_{t}\right)_{t \in[0, \cdot\rangle},[0, \cdot\rangle \subseteq \mathbb{R}$, on $X$ by the rule

$$
x \sim_{t} x^{\prime} \Leftrightarrow \rho\left(x, x^{\prime}\right) \leq \frac{1}{1+t}
$$


such that $\sim_{0}=X \times X$, and, if $\rho$ is an ultrametric, then $\cap_{t \in[0, \cdot\rangle} \sim_{t}=\Delta_{X}$. (In two other cases, i.e., if $1<\operatorname{diam}(X, \rho)<\infty$ or $\operatorname{diam}(X, \rho)=\infty$, the family $\left(\sim_{t}\right)$ is defined according to [16], Remark $\left.5(\mathrm{a})\right)$. Let, for every $t \in[0, \cdot\rangle$, $X_{t} \equiv X / \sim_{t}$ be the quotient set, and let $p_{t}: X \rightarrow X_{t}, p_{t}(x)=[x]_{t}$, be the quotient function. Observe that

$$
\rho\left(x, x^{\prime}\right)=0 \Leftrightarrow(\forall t \in[0, \cdot\rangle) \quad x \sim_{t} x^{\prime} .
$$

Hence,

$$
x \sim_{t} x^{\prime} \nRightarrow x \sim_{\rho} x^{\prime}\left(\Leftrightarrow \rho\left(x, x^{\prime}\right)=0\right),
$$

and thus, $\sim_{t}$ is not, generally, a congruence on $(X, \rho)$. Nevertheless, the following facts hold.

Lemma 6.10. For every $t \in[0, \cdot\rangle$, the rule

$$
d_{t}\left(\left[x_{1}\right]_{t},\left[x_{2}\right]_{t}\right)=\left\{\begin{array}{l}
0, \quad\left[x_{1}\right]_{t}=\left[x_{2}\right]_{t} \\
\rho\left(x_{1}, x_{2}\right),\left[x_{1}\right]_{t} \neq\left[x_{2}\right]_{t}
\end{array}\right.
$$

defines a function $d_{t}: X_{t} \times X_{t} \rightarrow \mathbb{R}$. Moreover, $\left(X_{t}, d_{t}\right)$ is an ultrametric space, and the quotient function $p_{t}:(X, \rho) \rightarrow\left(X_{t}, d_{t}\right)$ is continuous.

Proof. Given a $t \in[0, \cdot\rangle$, let $x_{1}, x_{1}^{\prime}, x_{2}, x_{2}^{\prime} \in X$ such that $\left[x_{1}\right]_{t}=\left[x_{1}^{\prime}\right]_{t}$ and $\left[x_{2}\right]_{t}=\left[x_{2}^{\prime}\right]_{t}$. This means that $x_{1} \sim_{t} x_{1}^{\prime}$ and $x_{2} \sim_{t} x_{2}^{\prime}$, and thus, $\rho\left(x_{1}, x_{1}^{\prime}\right) \leq$ $\frac{1}{1+t}$ and $\rho\left(x_{2}, x_{2}^{\prime}\right) \leq \frac{1}{1+t}$. Now, if $\left[x_{1}\right]_{t}=\left[x_{2}\right]_{t}$, then $\left[x_{1}^{\prime}\right]_{t}=\left[x_{2}^{\prime}\right]_{t}$. So, in this case the definition is correct. In the remaining case, i.e., if $\left[x_{1}\right]_{t} \neq\left[x_{2}\right]_{t}$, then $\left[x_{1}^{\prime}\right]_{t} \neq\left[x_{2}^{\prime}\right]_{t}$, i.e., $x_{1} \chi_{t} x_{2}$ and $x_{1}^{\prime} \chi_{t} x_{2}^{\prime}$, and thus, $\rho\left(x_{1}, x_{2}\right)>\frac{1}{1+t}$ and $\rho\left(x_{1}^{\prime}, x_{2}^{\prime}\right)>\frac{1}{1+t}$. Since $\rho$ is an ultrapseudometric, the strengthening of the triangle inequality implies that

$$
\rho\left(x_{1}, x_{2}\right)=\rho\left(x_{1}, x_{2}^{\prime}\right)=\rho\left(x_{1}^{\prime}, x_{2}^{\prime}\right),
$$

which proves that $d_{t}$ is well defined in this case as well. Now one straightforwardly verifies that $d_{t}$ is an ultrapseudometric on $X_{t}$. Moreover, if $d_{t}\left(\left[x_{1}\right]_{t},\left[x_{2}\right]_{t}\right)=0$, then or $\left[x_{1}\right]_{t}=\left[x_{2}\right]_{t}$, or $\rho\left(x_{1}, x_{2}\right)=0$ and $\left[x_{1}\right]_{t} \neq\left[x_{2}\right]_{t}$. However, the second case cannot occur. Indeed, if $\rho\left(x_{1}, x_{2}\right)=0$ would hold, then $x_{1} \sim_{t^{\prime}} x_{2}$ for all $t^{\prime}$, especially implying $\left[x_{1}\right]_{t}=\left[x_{2}\right]_{t}$ - a contradiction. Consequently, $d_{t}$ is an ultrametric. The continuity of $p_{t}$ follows by the definition of $d_{t}$.

Observe that

$$
t \leq t^{\prime} \Leftrightarrow \sim_{t^{\prime}} \subseteq \sim_{t} \Leftrightarrow \text { (the refinement) } \Leftrightarrow\left|X_{t}\right| \leq\left|X_{t^{\prime}}\right| .
$$

Then the induced function $p_{t t^{\prime}}:\left(X_{t^{\prime}}, d_{t^{\prime}}\right) \rightarrow\left(X_{t}, d_{t}\right), p_{t t^{\prime}} p_{t^{\prime}}=p_{t}$, is obviously continuous. Further, $\left(X_{0}, d_{0}\right)$ is a singleton, and, for every $t \in[0, \cdot\rangle$,

$$
x \sim_{\rho} x^{\prime} \Rightarrow x \sim_{t} x^{\prime},
$$


and thus, $\left|X_{t}\right| \leq\left|X_{\rho}\right|$. (Hence, each term $X_{t}$ belongs to the complementary part - comparing to the case with congruences - of the "quotient system" associated with $(X, \rho)$.) In this way we have constructed a morphism

$$
\boldsymbol{p}=\left(p_{t}\right):(X, \rho) \rightarrow(\boldsymbol{X}, \boldsymbol{d})=\left(\left(X_{t}, d_{t}\right), p_{t t^{\prime}},[0, \cdot\rangle\right)
$$

of pro-UPMet, associated with $(X, \rho) \in O b(U P M e t)$, such that $(\boldsymbol{X}, \boldsymbol{d})$ belongs to pro-UMet. Finally, observe that a needed "ultra" analogue of Lemma 7 holds as well. More precisely, if $|Y| \leq\left|X_{\rho}\right|$, then every continuous (admissible) $f:(X, \rho) \rightarrow(Y, \delta)$ uniquely continuously (admissible) factorizes through an $\left(X_{t}, d_{t}\right)$ and $\left|X_{t}\right| \leq|Y|$.

We now proceed as follows. Let $\kappa$ be an infinite cardinal, and let $U P M e t_{\kappa_{*}}\left(U P M e t_{\kappa^{*}}\right)$ be the full subcategory of $U P M e t$ determined by all the objects $(X, \rho)$ satisfying $\left|X_{\rho}\right|<\kappa\left(\left|X_{\rho}\right| \leq \kappa\right)$. Let $U_{\text {Met }} \kappa_{\kappa^{-}}\left(\right.$UMet $\left._{\kappa}\right)$ denote, as usually, the full subcategory of $U$ Met determined by all the objects having cardinalities less than (less or equal to) $\kappa$. Then, clearly, $U M e t_{\kappa^{-}} \subseteq U P M e t_{\kappa_{*}}\left(U M e t_{\kappa} \subseteq U P M e t_{\kappa^{*}}\right)$ is a full subcategory. The same holds in the admissible case, with the appropriate notation, as well. Let $T_{\kappa^{-}}$ $\left(T_{\kappa}\right)$ be the subset of $[0, \cdot\rangle$ such that, for every $t \in T_{\kappa^{-}}\left(t \in T_{\kappa}\right),\left|X_{t}\right|<\kappa$ $\left(\left|X_{t}\right| \leq \kappa\right)$, and let

$$
\boldsymbol{p}_{\kappa^{-}}:(X, \rho) \rightarrow(\boldsymbol{X}, \boldsymbol{d})_{\kappa^{-}}\left(\boldsymbol{p}_{\kappa}:(X, \rho) \rightarrow(\boldsymbol{X}, \boldsymbol{d})_{\kappa}\right)
$$

be the appropriate restriction of $\boldsymbol{p}$ to $T_{\kappa^{-}}\left(T_{\kappa}\right)$. Then, for every $(X, \rho)$ of $U P \operatorname{Met}_{\kappa_{*}}\left(U P M e t_{\kappa^{*}}\right)$, the proven facts imply that

- $\boldsymbol{p}_{\kappa^{-}}:(X, \rho) \rightarrow(\boldsymbol{X}, \boldsymbol{d})_{\kappa^{-}}$is a totally ordered epimorphic expansion with respect to $U M e t_{\kappa^{-}}$and to $U M e t_{\kappa^{-}}^{a}$;

- $\left(\boldsymbol{p}_{\kappa}:(X, \rho) \rightarrow(\boldsymbol{X}, \boldsymbol{d})_{\kappa}\right.$ is a totally ordered epimorphic expansion with respect to $U M e t_{\kappa}$ and to $\left.U M e t_{\kappa}^{a}\right)$;

- $p:(X, \rho) \rightarrow\left(X_{\rho}, d_{\rho}\right)$ is a rudimentary epimorphic expansion with respect to $U M e t$ and to $U M e t^{a}$;

- the index set $[0, \cdot\rangle \subseteq \mathbb{R}$ is sufficiently large to cover all the (infinite) cases.

Hence, the following theorem (in the categorical terms) holds true.

THEOREM 6.11. (i) For every infinite cardinal $\kappa$, the subcategories $U M e t_{\kappa^{-}} \subseteq U P M e t_{\kappa_{*}}$ and $U M e t_{\kappa} \subseteq U P M e t_{\kappa^{*}}\left(U M e t_{\kappa^{-}}^{a} \subseteq U P M e t_{\kappa_{*}}^{a}\right.$ and $U M e t_{\kappa}^{a} \subseteq U P M e t_{\kappa^{*}}^{a}$ ) are pro-reflective by means of epimorphic expansions indexed by totally ordered index sets of cardinalities less or equal $\aleph_{1}(\mathrm{CH})$.

(ii) The subcategory UMet $\subseteq$ UPMet $\left(U_{M}\right.$ Met $\left.^{a} \subseteq U_{P M e t^{a}}\right)$ is proreflective by means of rudimentary epimorphic expansions.

Hereby we shall not explicitly mention the appropriate quotient shape categories and corresponding functors. 
Corollary 6.12. For every infinite cardinal $\kappa_{0}$ and every infinite cardinal $\kappa<\kappa_{0}\left(\kappa \leq \kappa_{0}\right)$, every ultrapseudometric space $(X, \rho)$, with $\left|X_{\rho}\right|<\kappa_{0}$ $\left(\left|X_{\rho}\right| \leq \kappa_{0}\right)$, admits a quotient ultrametric $\kappa^{-}$-expansion ( $\kappa$-expansion) with a totally ordered index set $T \subseteq[0, \cdot \subseteq \subseteq \mathbb{R}$.

The next example shows that those shape categories provide mutually different classifications of ultrapseudometric spaces (strictly coarser than the isomorphiness).

EXAMPLE 6.13. Let $(X, \rho)$ be an ultrapseudometric non-metric space such that the corresponding ultrametric quotient space $\left(X_{\rho}, d_{\rho}\right)$ has cardinality $\left|X_{\rho}\right|>\aleph_{0}$. Then, by the above construction and results, the rudimentary $U$ Met-shape of $(X, \rho), S h_{U M e t}(X, \rho)$, is not the isomorphism class of $(X, \rho)$ in UPMet. Further, both of them differ from the UMet-shapes $S h_{0}(X, \rho)$ and $S h_{\aleph_{0}}(X, \rho)$. Finally, $S h_{\underline{0}}(X, \rho) \neq S h_{\aleph_{0}}(X, \rho)$ as well.

We finish this section with the following simple corollary concerning the admissible cases.

Corollary 6.14. A pair $(X, \rho),(Y, \sigma) \in O b\left(U P M e t_{\kappa_{*}}\right)\left(O b\left(U_{P M e \kappa_{\kappa^{*}}}\right)\right)$ has the same admissible quotient shape type, for all appropriate $\kappa^{-}(\kappa)$, (admissible rudimentary quotient shape type) if and only if, the corresponding pair of metric spaces $\left(X_{\rho}, d_{\rho}\right),\left(Y_{\sigma}, d_{\sigma}\right)$ has the same cardinality.

Proof. As we already mentioned, $\operatorname{Met}^{a}\left((A, d),\left(A^{\prime}, d^{\prime}\right)\right)=\operatorname{Set}\left(A, A^{\prime}\right)$. Thus, the conclusion follows by Theorem 5 (i) (Theorem 4) and Theorem 2.

\section{The (QUOTIENT) SHAPES OF TOPOLOGICAL SPACES}

For every topological space $X$ (we stress the topological structure only when necessary) and every equivalence relation $\sim \equiv \lambda$ on the set $X$, the topological structure on the quotient set $X / \lambda$ is defined to be the finest (largest, maximal) one such that the quotient function $p: X \rightarrow X / \lambda$ is continuous, i.e., a $V \subseteq X / \lambda$ is open if and only if $p^{-1}[V] \subseteq X$ is open. So, there is no restriction to the set $\Lambda$ of all equivalence relations on the set $X$. Recall that, for every mapping (continuous function) $f: X \rightarrow Y$, the equivalence relation $\sim_{f}$ (by the fibers of $f$ ) induces the factorization $f=u p$, where

$$
p: X \rightarrow X / \sim_{f}, \quad u: X / \sim_{f} \rightarrow Y
$$

and $p$ is a surjection. By that, $u$ is a homeomorphism, if and only if $f$ is an identification. Further, for every commutative diagram (in Top)

$$
\begin{array}{ccc}
X & \stackrel{f}{\rightarrow} & Y \\
\varphi \downarrow & & \downarrow \psi, \\
Z & \stackrel{g}{\rightarrow} & W
\end{array}
$$


there exists the following commutative diagram

$$
\begin{array}{cccccc}
X & \stackrel{p}{\rightarrow} & X / \sim_{f} & \stackrel{u}{\rightarrow} & Y \\
\varphi \downarrow & & \downarrow & \chi & & \downarrow \psi \\
Z & \stackrel{q}{\rightarrow} & Z / \sim_{g} & \stackrel{v}{\rightarrow} & W
\end{array} .
$$

By following the procedure of construction for Set (Section 4), one obtains the morphism $\boldsymbol{p}$ of pro-Top consisting of the appropriate quotient spaces and quotient mappings. Further, for every infinite cardinal $\kappa$, the corresponding restrictions $\boldsymbol{p}_{\kappa^{-}}$and $\boldsymbol{p}_{\kappa}$ of $\boldsymbol{p}$ yield the canonical $T o p_{\kappa^{--}}$and $T o p_{\kappa^{-}}$expansion of $X$, respectively. Thus, there exist the (quotient) shape categories of topological spaces, $S h_{\kappa^{-}}(T o p)$ and $S h_{\kappa}(T o p)$ (having the realizing categories proTop $\kappa^{-}$and pro-Top $\left.\kappa\right)$, and the shape functors $S_{\kappa^{-}}:$Top $\rightarrow S h_{\kappa^{-}}($Top $)$, $S_{\kappa}: T o p \rightarrow S h_{\kappa}(T o p)$ as well as the relating functor $S_{\kappa^{-} \kappa}: S h_{\kappa}(T o p) \rightarrow$ $S h_{\kappa^{-}}(T o p), S_{\kappa^{-}} S_{\kappa}=S_{\kappa^{-}}$, and also the relating functors $S_{\kappa \kappa^{\prime}}$, whenever $\kappa \leq \kappa^{\prime}$.

Notice that even in the general setting (the only condition is a cardinality) with an indiscrete codomain, a non-trivial result (different comparing to the Set-case) may occur. For instance, if $X, Y$ is a pair of non-homeomorphic spaces having the same cardinality, then, in general, the canonical map

$$
\operatorname{pro}^{-T o p}{ }_{\kappa}\left(\boldsymbol{X}_{\kappa}, \boldsymbol{Y}_{\kappa}\right) \rightarrow \operatorname{pro}_{-} \operatorname{Set}\left(U\left(\boldsymbol{X}_{\kappa}\right), U\left(\boldsymbol{Y}_{\kappa}\right)\right)
$$

( $U$ is the forgetful functor) does not reflect isomorphisms.

In order to obtain some more useful results, one has to consider the quotient shapes of topological space with respect to a specific "nice" full subcategory $\mathcal{D}_{\kappa} \subseteq$ Top. Clearly, then there should exist a directed subset $\Lambda_{\kappa} \subseteq \Lambda$ such that all the quotient spaces $X_{\lambda}, \lambda \in \Lambda_{\kappa}$, belong to $\mathcal{D}_{\kappa}$. However, the quotient spaces very rarely belong to a certain "nice" subcategory. Even for such a large class of Hausdorff spaces, $X / \lambda$ is Hausdorff provided $\lambda$ is closed (unavoidable) and $p: X \rightarrow X / \lambda$ is open. Nevertheless, a certain subcategory $\mathcal{D}$ of the full subcategory of all Hausdorff spaces $T 2 \subseteq$ Top could be pro-reflective, as well as the subcategories $\mathcal{D}_{\kappa^{-}}, \mathcal{D}_{\kappa}$ of $T 2_{\kappa^{-}}, T 2_{\kappa} \subseteq T 2$ respectively. Namely, for every mapping $f: X \rightarrow Y$ to a Hausdorff $Y$, the quotient space $X / \sim_{f}$ is Hausdorff (see also Lemmata $10-14$ and Theorem 7 below).

ExAmple 7.1. Let $X=\mathbb{R}$ (the Euclidean line). Since $\mathbb{R}$ is connected, every mapping $f: \mathbb{R} \rightarrow Y$ to a totally disconnected (especially, discrete, and thus Hausdorff) space $Y$ is constant. (Recall that all $\mathcal{D}$-expansion systems of a $\mathcal{C}$-object are mutually isomorphic in pro- $\mathcal{D}$; Section 2 ). Consequently, every $\boldsymbol{R}_{T 2_{0}}$-expansion system of $\mathbb{R}$ is isomorphic to the trivial rudimentary system $\lfloor\{*\}\rfloor$. In the same way, every $\boldsymbol{R}_{T 2 T D_{\kappa^{-}}}$-expansion $\left(\boldsymbol{R}_{T 2 T D_{\kappa}}\right.$-expansion $)$ system of $\mathbb{R}$ with respect to the full subcategory of totally disconnected Hausdorff spaces $(T 2 T D \subseteq T 2)$ is isomorphic to a trivial rudimentary system. Thus, those shapes of $\mathbb{R}$ are trivial (i.e., equal to the shape of a singleton). On the 
other hand, $S h_{T 2_{0}}(\mathbb{Q})$ and $S h_{T 2_{0}}(\mathbb{R} \backslash \mathbb{Q}$ ) are different and not trivial (compare Example 4 of Section 5 ). (The index set $\Lambda_{0}(\mathbb{Q})$ is uncountable, while $\Lambda_{\underline{0}}(\mathbb{R} \backslash \mathbb{Q})$ is countable.) In fact, it is not difficult to show that $\mathbb{R} \backslash \mathbb{Q}$ is $T 2_{\underline{0}}$-shape dominated by $\mathbb{Q}, S h_{T 2_{0}}(\mathbb{R} \backslash \mathbb{Q}) \leq S h_{T 2_{0}}(\mathbb{Q})$.

In order to get a better control over quotient spaces, we will select some special equivalence relations having a certain "degree of compatibility" with the topology. Let $\sim$ be an equivalence relation on a set $X$. Then there exists the induced function on the power set,

$$
2^{\sim}: 2^{X} \rightarrow 2^{X}, \quad 2^{\sim}(A) \equiv \tilde{A}=\cup_{a \in A}[a],
$$

where $[a]=\{x \mid x \in X \wedge x \sim a\}$ is the equivalence class of $a$. Obviously, $A \subseteq \tilde{A}$, and one can straightforwardly verifiy the following properties of $2^{\sim}$ :

(i) $\left(\cup_{j \in J} A_{j} \tilde{)}=\cup_{j \in J} \tilde{A}_{j}\right.$;

(ii) $(\tilde{A})=\tilde{A}$;

(iii) $\left(\cap_{j \in J} A_{j}\right) \subseteq \cap_{j \in J} \tilde{A}_{j}$ (if $\tilde{A}_{j}=A_{j}$ for every $j$, then the equality holds);

(iv) $\left(X \backslash A_{j}\right) \supseteq X \backslash \tilde{A}$ (the equality holds $\Leftrightarrow \tilde{A}=A$ );

(v) $(\forall A \subseteq X) \tilde{A}=p^{-1}[p[A]]$, where $p: X \rightarrow X / \sim, p(x)=[x]$.

(vi) $(\forall f: X \rightarrow Y)(\forall A \subseteq X) \tilde{A}^{f}=f^{-1}[f[A]]$.

Definition 7.2. Let $X$ be a topological space and let $\sim$ be an equivalence relation on $X$. If, for every $U \subseteq X$ open ( $F \subseteq X$ closed), $\tilde{U}$ is open ( $\tilde{F}$ is closed), then $\sim$ is said to be an open-(closed)-preserving equivalence relation on $X$. If $\sim$ is open-and closed-preserving, then it is said to be a congruence on the space $X$.

The next five lemmata follow immediately.

Lemma 7.3. For every topological space $(X, \mathcal{T})$. every basis $\mathcal{B}$ for $\mathcal{T}$ and every equivalence relation $\sim$ on $X$, the following assertions are mutually equivalent:

(i) $\sim$ is open-preserving;

(ii) $p: X \rightarrow X / \sim$ is open;

(iii) $(\forall W \in \mathcal{B}) \tilde{W} \in \mathcal{T}$.

(iv) $\left(\forall U \subseteq X\right.$ open) $\tilde{U}=p^{-1}[p[U]] \subseteq X$ is open.

Lemma 7.4. For every topological space $X$ and every equivalence relation $\sim$ on $X$, the following assertions are mutually equivalent:

(i) $\sim$ is closed-preserving;

(ii) $p: X \rightarrow X / \sim$ is closed;

(iii) $\left(\forall F \subseteq X\right.$ closed) $\tilde{F}=p^{-1}[p[F]] \subseteq X$ is closed.

LEMma 7.5. For every topological space $X$ and every equivalence relation $\sim$ on $X$, the following assertions are mutually equivalent:

(i) is a congruence; 
(ii) $p: X \rightarrow X / \sim$ is open and closed;

(iii) $\left(\forall A \subseteq X\right.$ open and closed) $\tilde{A}=p^{-1}[p[A]] \subseteq X$ is open and closed.

Lemma 7.6. Let $f: X \rightarrow Y$ be a mapping of topological spaces, and let $\sim_{f}$ be induced by fibers of $f\left(f^{-1}[\{y\}], y \in Y\right)$.

(i) If $f$ is open, then $\sim_{f}$ is open-preserving, and the converse holds whenever $f$ is an identification;

(ii) If $f$ is closed, then $\sim_{f}$ is closed-preswerving, and the converse holds whenever $f$ is an identification;

(iii) If $f$ is open and closed, then $\sim_{f}$ is a congruence, and the converse holds whenever $f$ is an identification;

(iv) $f$ is open (closed) if and only if, $p: X \rightarrow X / \sim_{f}$ and $g=" f p^{-1}$ " are open (closed).

LEMMA 7.7. If $\sim$ and $\sim^{\prime}$ on a space $X$ are open-preserving (closedpreserving, congruences), then so is $\sim \cap \sim^{\prime}$ refining the both.

THEOREM 7.8. The category $T_{2}$ op of Hausdorff spaces is a pro-reflective subcategory of Top. The same is true for their restrictions to mappings that are open and closed.

Proof. For every topological space, there exists a congruence $\sim$ on $X$ (at least, the coarsest equivalence relation $\sim=X \times X$ is a congruence). Since, for every pair of infinite cardinals $\kappa, \kappa^{\prime}$,

$$
\kappa \cdot \kappa^{\prime}=\max \left\{\kappa, \kappa^{\prime}\right\}
$$

holds, the conclusion follows by Lemma 13. In the case of the restrictions, apply also Lemma 11.

EXAMPLE 7.9. Let

$$
X=\left\{\frac{n}{n+1} \mid n \in\{0\} \cup \mathbb{N}\right\} \cup\{1\}
$$

and

$$
Y=\{(-1,0)\} \cup\left(\bigcup_{n \in \mathbb{N}}\left\{-\frac{n}{n+1}\right\} \times \mathbb{R}\right) \cup(X \times\{0\})
$$

be topological spaces carring the Euclidean subspace topologies. We shall prove that $X$ and $Y$ have the same finite $T_{2}$-shape, i.e., that $S h_{0}(X)=$ $S h_{\underline{0}}(Y)$, where $S h_{\underline{0}}$ is the abbreviation for $S h_{\underline{0}}\left(T o p, T_{2} o p\right)$. Let

$$
\boldsymbol{p}_{\underline{0}}=\left(p_{\lambda}\right): X \rightarrow \boldsymbol{X}_{\underline{0}}=\left(X_{\lambda}, p_{\lambda \lambda^{\prime}}, \Lambda_{\underline{0}}\right)
$$

and

$$
\boldsymbol{q}_{\underline{0}}=\left(q_{\mu}\right): Y \rightarrow \boldsymbol{Y}_{\underline{0}}=\left(Y_{\mu}, q_{\mu \mu^{\prime}}, M_{\underline{0}}\right)
$$

be the canonical $T_{2} o p_{0}$-expansions of $X$ and $Y$ respectively. Observe that the terms

$$
X_{\lambda(k)}=\left\{\{0\}, \ldots,\left\{\frac{k-1}{k}\right\},[1]_{k}\right\}, k \in \mathbb{N}
$$


of $\boldsymbol{X}_{\underline{0}}$, where

$$
[1]_{k}=\left\{\frac{n}{n+1} \mid n \geq k \in \mathbb{N}\right\} \cup\{1\},
$$

determine an inverse sequence $\boldsymbol{X}_{\underline{0}}^{(k)}$ which is is cofinal subsystem of $\boldsymbol{X}_{\underline{0}}$. Moreover, the inverse subsequence $\boldsymbol{X}_{0}^{(2 k)}$ of $\boldsymbol{X}_{0}^{(k)}$ determined by terms $X_{\lambda(2 k)}$ is cofinal in $\boldsymbol{X}_{\underline{0}}$ as well. Obviously, $X_{\lambda(2 k)}$ is a discrete space consisting of $2 k+1$ points, and thus, it is homeomorphic to $\{1,2, \ldots, 2 k, 2 k+1\} \subseteq \mathbb{N}$. Similarly, since every $\left\{-\frac{n}{n+1}\right\} \times \mathbb{R}$ is connected, the terms

$$
Y_{\mu(k)}=\left\{[(-1,0)]_{k},\left[\left(-\frac{k-1}{k}, 0\right)\right]_{k}, \ldots,\{(0,0)\}, \ldots,\left\{\left(\frac{k-1}{k}, 0\right)\right\},[(1,0)]_{k}\right\},
$$

for $k \in \mathbb{N}$ of $\boldsymbol{Y}_{\underline{0}}$, where

$$
[(-1,0)]_{k}=\{(-1,0)\} \cup\left(\bigcup_{n \geq k}\left\{-\frac{n}{n+1}\right\} \times \mathbb{R}\right)
$$

and

$$
\left[\left(-\frac{k-1}{k}, 0\right)\right]_{k}=\left\{-\frac{k}{k+1}\right\} \times \mathbb{R}
$$

determine an inverse sequence $\boldsymbol{Y}_{\underline{0}}^{(k)}$ which is is cofinal subsystem of $\boldsymbol{Y}_{\underline{0}}$. Clearly, $Y_{\mu(k)}$ is homeomorphic to $\{1,2, \ldots, 2 k, 2 k+1\} \subseteq \mathbb{N}$ as well. The conclusion follows straightforwardly.

In order to investigate the quotient shapes of some special classes of topological spaces, one can choose, by means of the above lemmata, various suitable subcategories of Top (by reducing Mor (Top) if necessary) and their proreflective "nice" subcategories. One of the most interesting of those classes could be that of all completely regular spaces (or Tychonoff or $T_{3.5}$-spaces). Namely, the complete regularity is the same as a (topological) gauge structure, while every gauge structure is a separating family of pseudometrics (see [5], Chapter IX, Section 10). So there is a firm relation to the results of Section 6. Also, a countable gauge structure yields a metric space. Further on this line is the subcategory of all uniform spaces. We postpone this job for the future.

\section{THE SHAPES OF MONOIDS}

Let $M$ on denote the category of all monoids $(M, \cdot)$ (associative groupoids with neutral elements) and their homomorphisms

$$
f:(M, \cdot) \rightarrow(N, \circ), \quad f(x \cdot y)=f(x) \circ f(y) \wedge f\left(e_{M}\right)=e_{N} .
$$

(In the sequel, we shall often omit the operations "." and "०".) In order to consider the quotients of a monoid, the appropriate equivalence relations - congruences are needed, such that the quotient sets (functions) become monoids (homomorphisms). Given a monoid $(M, \cdot)$, an equivalence relation $\sim \equiv \lambda$ on the set $M$ is said to be a congruence on the monoid if the following condition is fulfilled: 
(1) $\left(\forall x, y, x^{\prime}, y^{\prime} \in M\right)\left(x \sim x^{\prime} \wedge y \sim y^{\prime}\right) \Rightarrow\left(x y \sim x^{\prime} y^{\prime}\right)$.

It that case the quotient set $M / \lambda$, endowed with the operation $[x][y]=[x y]$, becomes a monoid having for the unique neutral element the class $[e]$ (which is a submonoid of $(M, \cdot)$ ) of the neutral element $e \in M$, and the quotient function $p: M \rightarrow M / \lambda, p(x)=[x]$ becomes a homomorphism.

If $f: M \rightarrow N$ is a homomorphism of monoids, then the equivalence relation $\sim_{f}$ induced by fibers of $f$ (i.e., by the kernel of $f$ ) is a congruence on $M$. Indeed, if $x_{1} \sim_{f} x_{1}^{\prime}$ and $x_{2} \sim_{f} x_{2}^{\prime}$, i.e., $f\left(x_{1}\right)=f\left(x_{1}^{\prime}\right)$ and $f\left(x_{2}\right)=f\left(x_{2}^{\prime}\right)$, then

$$
f\left(x_{1} x_{2}\right)=f\left(v_{1}\right) f\left(x_{2}\right)=f\left(x_{1}^{\prime}\right) f\left(x_{2}^{\prime}\right)=f\left(x_{1}^{\prime} x_{2}^{\prime}\right),
$$

i.e., $x_{1} x_{2} \sim_{f} x_{1}^{\prime} x_{2}^{\prime}$, and (1) is fulfilled. Finally, if $\sim$ and $\sim^{\prime}$ are congruences on $M$, then one readily sees that so is

$$
\sim^{\prime \prime}=\sim \cap \sim^{\prime}\left([x]^{\prime \prime}=[x] \cap[x]^{\prime}\right)
$$

because property (1) is preserved under intersection. In addition, if $\sim^{\prime} \equiv \lambda^{\prime}$ refines $\sim \equiv \lambda, \lambda^{\prime} \geq \lambda$, the relating quotient function

$$
\tilde{p}: M / \lambda^{\prime} \rightarrow M / \lambda,[x]^{\prime} \mapsto[x],
$$

$\left(\tilde{p} p^{\prime}=p\right)$ is a homomorphism:

$$
\tilde{p}\left([x]^{\prime}[y]^{\prime}\right)=\tilde{p}\left([x y]^{\prime}\right)=[x y]=[x][y]=\tilde{p}\left([x]^{\prime}\right) \tilde{p}\left([y]^{\prime}\right) .
$$

Hence, from now on, the quotient shape theory for monoids can be developed by the general procedure of Section 3. Thus, given a monoid $M$ and an infinite cardinal $\kappa$, the corresponding canonical $\kappa^{-}$-expansion ( $\kappa$-expansion) is fully determined by the quotients having cardinalities less than (less or equal to) $\kappa$. Clearly, if $\kappa>|M|$, then $\boldsymbol{p}_{\kappa^{-}}$is isomorphic to the rudimentary expansion of $M$, while $\kappa \geq|M|$ implies that $\boldsymbol{p}_{\kappa}$ is isomorphic to the rudimentary expansion of $M$. We will not repeat the construction of the appropriate shape categories and functors any more.

EXAMPLE 8.1. (a) Let $(\mathbb{N}, \cdot)$ be the multiplicative monoid on the set $\mathbb{N}$ of natural numbers. Let, for each $m \in \mathbb{N}$, the relation $\sim^{m}$ on $\mathbb{N}$ be defined by

$$
n \sim^{m} n^{\prime} \Leftrightarrow(\exists l \in \mathbb{Z}) \quad n^{\prime}=n m^{l} .
$$

Then $\sim^{m}$ is an equivalence relation on $\mathbb{N}\left(n=n^{\prime} m^{-l}\right)$, and $\sim^{1}=\Delta_{\mathbb{N}}$. Moreover, it is a congruence on $(\mathbb{N}, \cdot)$ because $n_{1}^{\prime}=n_{1} m^{l_{1}}$ and $n_{2}^{\prime}=n_{2} m^{l_{2}}$ imply

$$
n_{1}^{\prime} n_{2}^{\prime}=n_{1} m^{l_{1}} n_{2} m^{l_{2}}=\left(n_{1} n_{2}\right) m^{l_{1}+l_{2}} .
$$

The congruence class $[n]^{m}$ of an $n \in \mathbb{N}$ is the set

$$
\left\{n m^{l} \mid l \in\{0\} \cup \mathbb{Z}\right\} \cap \mathbb{N} .
$$

If $n \leq m>1$, then $[n]^{m}$ is countably infinite. However, for every $m$, the quotient monoid $\left(\mathbb{N} / \sim^{m}, \cdot\right)$ is countably infinite too. 
(b) Let us find out a Mon $_{\underline{0}^{-}}$expansion of $(\mathbb{N}, \cdot)$. Consider a congruence $\sim \equiv \lambda$ on $(\mathbb{N}, \cdot)$ such that $|\mathbb{N} / \lambda|<\aleph_{0}$. If $|\mathbb{N} / \lambda|=1$, then $\lambda=\mathbb{N} \times \mathbb{N}$. If $|\mathbb{N} / \lambda|=2$, then the two following possibilities occur:

or $\lambda$ corresponds to the partition $\{1\}, \mathbb{N} \backslash\{1\}$ (if a monoid $M$ has no invertible element except $e=e^{-1}$, then $x \sim y \Leftrightarrow((x=y) \vee(x, y \in$ $M \backslash\{e\}))$ is a congruence);

or $\lambda$ corresponds to the partition $2 \mathbb{N}-\mathbf{1}, 2 \mathbb{N}$, i.e., it is the congruence

$$
n \sim_{2} n^{\prime} \Leftrightarrow n \equiv n^{\prime}(\bmod 2) .
$$

Furthermore, for every $m \geq 2$, the equivalence relation $\sim_{m}$,

$$
n \sim_{m} n^{\prime} \Leftrightarrow n \equiv n^{\prime}(\bmod m)
$$

is a congruence on $(\mathbb{N}, \cdot)$ such that $\left|\mathbb{N} / \sim_{m}\right|=m$. In that case,

$$
\left(\mathbb{N} / \sim_{m}, \cdot\right) \cong\left(N_{m}, \cdot m\right),
$$

where the congruence classes

$$
[n]_{m}=\{n, n+m, \ldots, n+k m, \ldots\}, \quad n \in \mathbb{N},
$$

are represented by $n \in\{1, \ldots, m\}$, and $[n]_{m} \cdot_{m}\left[n^{\prime}\right]_{m}$ is defined as usual (residuum 0 is identified with $m$ ). Another type of congruence on $(\mathbb{N}, \cdot$ ), that induces a finite quotient monoid, is $\sim_{m}^{\prime}$ which corresponds to the partition

$$
\{1\}, \ldots,\{m\},\{m+1, m+2, \ldots\} \text {. }
$$

(Notice that $n>m$ implies $n k \in\{m+1, m+2, \ldots\}$, for every $k$.) Finally, a slightly deeper analysis shows the following: For every congruence $\lambda$ on $(\mathbb{N}, \cdot)$ such that $2<|\mathbb{N} / \lambda|<\aleph_{0}$, there exists a finer congruence of the type $\sim_{m} \cap \sim_{m}^{\prime}, m \in \mathbb{N}$. Its quotient set consists of $2 m$ congruence classes. The corresponding subset $\Lambda_{\underline{0}}^{*} \equiv\left(\mathbb{N}, \leq^{*}\right) \subseteq \Lambda_{\underline{0}}$ (ordered by divisibility; see Example 1 (a) of Section 5) is cofinal in $\Lambda_{\underline{0}}$. Consequently, the restriction

$$
\boldsymbol{p}_{\underline{0}}^{*}=\left(p_{m}\right):(\mathbb{N}, \cdot) \rightarrow(\boldsymbol{N}, \cdot)_{\underline{0}}^{*}=\left(\left(N_{m}, \cdot\right), p_{m m^{\prime}},\left(\mathbb{N}, \leq^{*}\right)\right)
$$

(of the canonical Mon $_{\underline{0}}$-expansion $\boldsymbol{p}_{\underline{0}}$ ) is a (countable) Mon $_{\underline{0}}$-expansion of the monoid $(\mathbb{N}, \cdot)$. It is a well known fact ([9], VIII., Ex. A. 1.; [18], Lemma 9) that it is isomorphic to an inverse sequence. Thus, for instance,

$$
\boldsymbol{p}_{\underline{0}}^{\prime}=\left(p_{m}^{\prime}\right):(\mathbb{N}, \cdot) \rightarrow(\boldsymbol{N}, \cdot)_{\underline{0}}^{\prime}=\left(\left(N_{m}^{\prime}, \cdot\right), p_{m m^{\prime}}^{\prime}, \mathbb{N}\right),
$$

where $N_{m}^{\prime}=N_{m !}, p_{m m^{\prime}}^{\prime}=p_{m ! m^{\prime} !}, p_{m}^{\prime}=p_{m !}$, is a sequential Mon $_{\underline{0}}$-expansion of $(\mathbb{N}, \cdot)$.

EXAMPLE 8.2. Let $(\mathbb{Z},+)$ be the additive monoid of integers, and let $\sim$ be a congruence on $(\mathbb{Z},+)$ such that the quotient monoid $(\mathbb{Z} / \sim,+)$ is finite. An analysis shows $((\mathbb{Z},+)$ has invertible elements! $)$ that $\sim=\sim_{m}$, for some $m \in \mathbb{N}$, i.e., that

$$
k \sim_{m} k^{\prime} \Leftrightarrow k \equiv k^{\prime}(\bmod m) .
$$


The congruence classes

$$
[k]_{m}=\{k, k \pm m, \ldots, k \pm n m, \ldots\}, \quad k \in \mathbb{Z},
$$

are represented by $k \in\{0, \ldots, m-1\}$, while

$$
\left(\mathbb{Z} / \sim_{m},+\right) \cong\left(\{0, \ldots, m-1\},+\equiv+_{m}\right) \equiv\left(\mathbb{Z}_{m},{ }{ }_{m}\right) .
$$

Thus, the canonical Mon $_{0}$-expansion of $(\mathbb{Z},+)$ is

$$
\boldsymbol{q}_{\underline{0}}=\left(q_{M}\right):(\mathbb{Z},+) \rightarrow(\boldsymbol{Z},+)_{\underline{0}}=\left(\left(\mathbb{Z}_{m},{ }_{m}\right), q_{m m^{\prime}},\left(\mathbb{N}, \leq^{*}\right)\right) .
$$

Let $(\{0\} \cup \mathbb{N},+)$ be the additive monoid of non-negative integers, which is a proper (commutative) submonoid of $(\mathbb{Z},+)$ having no invertible element except 0 . Then the congruences $\sim_{m}$ on $(\mathbb{Z},+)$ restricted to $\{0\} \cup \mathbb{N}$ are also congruences on $(\{0\} \cup \mathbb{N},+)$ providing the (same) finite quotient monoids $\left((\{0\} \cup \mathbb{N}) / \sim_{m},+\right) \equiv\left(\mathbb{N}_{m},+_{m}\right) \cong\left(\mathbb{Z}_{m},+_{m}\right)$ of cardinality $m$. Further, another type of congruence on $(\{0\} \cup \mathbb{N},+)$, that induces a finite quotient, is $\sim_{m}^{\prime}$ corresponding to the partition

$$
\{0\}, \ldots,\{m-1\},\{m, m+1, \ldots\}, \quad m \in \mathbb{N}
$$

$\left(\sim_{0}^{\prime}=(\{0\} \cup \mathbb{N}) \times(\{0\} \cup \mathbb{N})\right)$. An analysis shows that the family of all intersections $\sim_{m} \cap \sim_{m}^{\prime} \equiv \lambda_{m}, m \in \mathbb{N}$, is cofinal in the family of all congruences on $(\{0\} \cup \mathbb{N},+)$ providing finite quotients. The quotient monoid $(\{0\} \cup \mathbb{N}) / \lambda_{m} \equiv N_{m}^{0}$ consists of all intersections of the appropriate congruence classes, i.e.,

$$
\left(N_{m}^{0},+\right) \equiv\left(\left\{[n]_{m} \cap[n]_{m}^{\prime} \mid n \in\{0\} \cup \mathbb{N}\right\},+\right),
$$

where

$$
[n]_{m} \cap[n]_{m}^{\prime}=\left\{\begin{array}{c}
\{n\}, \quad 0 \leq n \leq m-1 \\
\{n, n+m, n+2 m, \ldots\}, m \leq n \leq 2 m-1
\end{array}\right.
$$

and $\left|\left(N_{m}^{0},+\right)\right|=2 m$. Therefore, the canonical Mon $_{\underline{0}}$-expansion of $(\{0\} \cup \mathbb{N},+)$ admits a restriction

$$
\boldsymbol{r}_{\underline{0}}=\left(r_{m}\right):(\{0\} \cup \mathbb{N},+) \rightarrow\left(N^{0},+\right)_{\underline{0}}=\left(\left(N_{m}^{0},+\right), r_{m m^{\prime}},\left(\mathbb{N}, \leq^{*}\right)\right)
$$

that is a Mon $_{\underline{0}}$-expansion of $(\{0\} \cup \mathbb{N},+)$ too. Similarly to Example 13 (b), there exists a sequential $M_{0} n_{0}$-expansion of $(\{0\} \cup \mathbb{N},+)$ that is cofinal in $\boldsymbol{r}_{0}$. Finally, observe that, for every $m \in \mathbb{N}$, the canonical monomorphism (inclusion) $j_{m}:\left(\mathbb{Z}_{m},+_{m}\right) \rightarrow\left(N_{m}^{0},+\right)$ is a section, i.e., it admits a left inverse (retraction) $\rho_{m}:\left(N_{m}^{0},+\right) \rightarrow\left(\mathbb{Z}_{m},+_{m}\right), \rho_{m} j_{m}=1_{\mathbb{Z}_{m}}$, by putting $\rho_{m}(\{n\})=$ $n, n \in\{0, \ldots, m-1\}$, and $\rho_{m}(\{n+k m \mid k \in\{0\} \cup \mathbb{N}\})=n-m$ (or $\left.=0\right)$, $n \in\{m, \ldots, 2 m-1\}$. Moreover, they extend to inverse systems, i.e., there exist

$$
j:(Z,+)_{\underline{0}} \rightarrow\left(N^{0},+\right)_{\underline{0}}, \quad \rho:\left(N^{0},+\right)_{\underline{0}} \rightarrow\left(\mathbb{Z}_{0},+\right)_{\underline{0}}
$$

of pro-Mon $\underline{0}$ such that $\boldsymbol{\rho j}=\mathbf{1}_{(\boldsymbol{Z},+)_{0}}$. Hence, $(\mathbb{Z},+)$ is finitely shape dominated by its submonoid $(\{0\} \cup \mathbb{N},+), S h_{\underline{0}}(\mathbb{Z},+) \leq S h_{\underline{0}}(\{0\} \cup \mathbb{N},+)$ (of course, 
neither $(\mathbb{Z},+)$ is dominated, in Mon, by $(\{0\} \cup \mathbb{N},+)$, nor $(\{0\} \cup \mathbb{N},+)$ is dominated by $(\mathbb{Z},+) !)$.

REMARK 8.3. We have not considered the quotient shapes in the categories of groupoids and semigroups. Nevertheless, for the sake of completeness, the only condition for a congruence in such a (sub)category is property (1) as well.

\section{THE SHAPES OF GROUPS}

9.1. The general case. As in the previous section (concerning monoids), an equivalence relation $\sim$ on a group $(G, \cdot)$ is said to be a congruence if

(1) $\left.\left(\forall x, x^{\prime}, y, y^{\prime} \in G\right)\left(x \sim x^{\prime} \wedge y \sim y^{\prime}\right) \Rightarrow x y \sim x^{\prime} y^{\prime}\right)$.

It is well known that a congruence $\sim$ is induced by a normal $\operatorname{subgroup}(N, \cdot) \unlhd$ $(G, \cdot)$ and that $N=[e]$. We denote, as usually, the quotient group $\mathrm{G} / \sim$ by $G / N$, and by $p: G \rightarrow G / N$ the quotient homomorphism. If $\Lambda$ is the set of all congruences $\lambda \equiv \sim$ on $G$, then we order $\Lambda$ by the refiniment. It corresponds to

$$
\lambda \leq \lambda^{\prime} \Leftrightarrow N_{\lambda^{\prime}} \leq N_{\lambda} \text { (the subgroup). }
$$

Since the intersection of any pair of normal subgroups is a normal subgroup, $\Lambda$ is directed as well. In the sequel, we shall abbreviate $G / N_{\lambda} \equiv G_{\lambda}$. If $f: G \rightarrow H$ is a homomorphism that preserves given congruences $\sim \equiv \lambda$ and $\sim^{\prime} \equiv \mu$, i.e.,

$$
x \sim x^{\prime} \Rightarrow f(x) \sim^{\prime} f\left(x^{\prime}\right)
$$

(equivalently, $\left.f\left(\left[e_{G}\right]\right) \subseteq\left[e_{H}\right]^{\prime}\right)$, then there exists a unique homomorphism $\tilde{f}: G_{\lambda} \rightarrow H_{\mu}, \tilde{f}[x]=[f(x)]^{\prime}$, i.e., $\tilde{f}\left(x\left[e_{G}\right]\right)=f(x)\left[e_{H}\right]^{\prime}$, such that the following diagram (in the category Grp)

$$
\begin{array}{ccc}
G_{\lambda} & \stackrel{p_{\lambda}}{\leftarrow} & G \\
\tilde{f} \downarrow & & \downarrow f \\
H_{\mu} & \stackrel{q_{\mu}}{\leftarrow} & H
\end{array}
$$

commutes, i.e., $\tilde{f} p_{\lambda}=q_{\mu} f$. Consequently, if $\lambda \leq \lambda^{\prime}$, then $1_{G}: G \rightarrow G$ is $\left(\lambda, \lambda^{\prime}\right)$-preserving, and thus, there exists a unique (effective) epimorphism $p_{\lambda \lambda^{\prime}}: G_{\lambda^{\prime}} \rightarrow G_{\lambda}$ such that $p_{\lambda \lambda^{\prime}} p_{\lambda^{\prime}}=p_{\lambda}$. Further, for every homomorphism $f: G \rightarrow H$, the equivalence relation $\sim_{f}$ induced by the fibers of $f, f^{-1}(y)$, $y \in H$, (i.e., by the kernel of $f$ ) is a congruence on $G$. Hence, there exists a $\lambda \in \Lambda$ such that $\sim_{f}=\lambda$ and there exists a unique homomorphism $f_{\lambda}$ : $G_{\lambda} \rightarrow H$ such that $f_{\lambda} p_{\lambda}=f$. If, in addition, $f$ is an epimorphism, then $f_{\lambda}$ is an isomorphism ("The first isomorphism theorem"). In this way we have obtained an inverse system

$$
\boldsymbol{G}=\left(G_{\lambda}, p_{\lambda \lambda^{\prime}}, \Lambda\right)
$$


in Grp, and a morphism

$$
\boldsymbol{p}=\left(p_{\lambda}\right): G \rightarrow \boldsymbol{G}
$$

of pro-Grp that is a Grp-expansion of $G$. However, there exists $\lambda_{*}=\max \Lambda$ (corresponding to $[e] \unlhd G$ ), and $G_{\lambda_{*}}=G$. Therefore, we have to reduce $\Lambda$ so that the restriction remains an expansion. Given an infinite cardinal $\kappa$, let $\Lambda_{\kappa^{-}}\left(\Lambda_{\kappa}\right)$ be the subset of $\Lambda$ consisting of all elements $\lambda$ such that $\left|G_{\lambda}\right|<\kappa$ $\left(\left|G_{\lambda}\right| \leq \kappa\right)$. Then the restriction of $\boldsymbol{p}$,

$$
\boldsymbol{p}_{\kappa^{-}}=\left(p_{\lambda}\right): G \rightarrow \boldsymbol{G}_{\kappa^{-}}=\left(G_{\lambda}, p_{\lambda \lambda^{\prime}}, \Lambda_{\kappa^{-}}\right)
$$

and

$$
\boldsymbol{p}_{\kappa}=\left(p_{\lambda}\right): G \rightarrow \boldsymbol{G}_{\kappa}=\left(G_{\lambda}, p_{\lambda \lambda^{\prime}}, \Lambda_{\kappa}\right)
$$

are (the canonical) $G r p_{\kappa^{-}}$-expansion and $G r p_{\kappa^{-}}$expansion of $G$, respectively. This shows that $G r p_{\kappa^{-}}$and $G r p_{\kappa}$ are pro-reflective subcategories of Grp (of course, in the case of a finite group $G$, one may choose, for every $\kappa^{-}$-and $\kappa$-expansion of $G$, the rudimentary identity expansion $\left.\left\lfloor 1_{G}\right\rfloor: G \rightarrow G\right)$.

Now one obtains, in the general way, the shape categories $S h_{\kappa^{-}}(G r p)$ and $S h_{\kappa}(G r p)$, and the shape functors $S_{\kappa^{-}}: G r p \rightarrow S h_{\kappa^{-}}(G r p)$ and $S_{\kappa}: G r p \rightarrow$ $S h_{\kappa}(G r p)$, as well as the relating functor $S_{\kappa^{-} \kappa}: S h_{\kappa}(G r p) \rightarrow S h_{\kappa^{-}}(G r p)$ such that $S_{\kappa^{-} \kappa} S_{\kappa}=S_{\kappa^{-}}$.

REMARK 9.1. The analogous theory holds in whole for the category $A b$ of Abelian groups as well. Observe that the above expansions of a group $G$ admit to consider a partial (or the full) normal system of $G$ (instead only a chain or a normal sequence).

REMARK 9.2. According to [14], Theorem 1.6, there exists a bijection

$$
S h_{\underline{0}}(G, H) \approx \operatorname{LocGrp}\left(\lim \left\{\operatorname{locgrp}\left(G_{\lambda}\right)\right\}, \lim \left\{\operatorname{locgrp}\left(H_{\mu}\right)\right\}\right),
$$

where lim is the inverse limit in LocGrp (the category of localic groups), while $\operatorname{locgrp}\left(G_{\lambda}\right)$ is the localic group associated with the finite group $G_{\lambda}$ carrying the discrete topology.

EXAMPLE 9.3. Let $\mathbb{Z}$ be the additive group of integers. Each of its subgroups is (normal) of the form $k \mathbb{Z}$, and the quotient group is $\left(\mathbb{Z}_{k},+_{k}\right)$, $k \in\{0\} \cup \mathbb{N}$. (The corresponding congruences $\sim_{k}$, see Example 14 of Section 8 , are defined by the rule

$$
m \sim_{k} m^{\prime} \Leftrightarrow m \equiv m^{\prime}(\bmod k),
$$

i.e., $m-m^{\prime}=l k$, for some $l \in \mathbb{Z}$, and $\sim_{k^{\prime}}$ refines $\sim_{k}$ whenever $k^{\prime}=r k$, for some $r \in \mathbb{N}$.) Thus, the canonical $G r p_{0}$-expansion of the group $\mathbb{Z}$ is

$$
\boldsymbol{p}_{\underline{0}}=\left(p_{k}\right): \mathbb{Z} \rightarrow \boldsymbol{Z}_{\underline{0}}=\left(Z_{k}, p_{k k^{\prime}}, \Lambda_{\underline{0}}=\left(\mathbb{N}, \leq^{*}\right)\right)
$$

(see Examples 1(a) and 6(a)), that is isomorphic to the sequential Grp $p^{-}$ expansion

$$
\boldsymbol{p}_{\underline{0}}^{\prime}=\left(p_{k !}\right): \mathbb{Z} \rightarrow \boldsymbol{Z}_{\underline{0}}^{\prime}=\left(Z_{k !}, p_{k ! k^{\prime} !}, \mathbb{N} !\right)
$$


Clearly, the canonical $\aleph_{0}$-expansion of $\mathbb{Z}$ reduces to the identity homomorphism.

EXAMPLE 9.4. (a) Let $(\mathbb{R},+)$ be the additive group of real numbers, and let $N$ be a subgroup of $(\mathbb{R},+)$. Then,

$$
|(\mathbb{R},+) / N|<\aleph_{0} \Rightarrow N=(\mathbb{R},+) .
$$

Therefore, the finite shape of $(\mathbb{R},+)$ is trivial. (The countable shape of $(\mathbb{R},+)$ is not trivial because, for every $n \in \mathbb{N}$, there exists a subgroup $N_{n}$ such that $(\mathbb{R},+) / N_{n}$ is isomorphic to $(\mathbb{Q},+)^{n}$. Namely, $(\mathbb{R},+)$ is an (uncountably infinite-dimensional vectorial space over $\mathbb{Q}$.) Finally, the canonical $\aleph_{1}$ expansion of $(\mathbb{R},+)$ reduces to the identity homomorphism. Since the multiplicative group $\left(\mathbb{R}^{+}, \cdot\right)$ is isomorphic to $(\mathbb{R},+)$, the same conclusion holds for it as well.

(b) Let $(\mathbb{R} \backslash\{0\}, \cdot)$ be the multiplicative group of non-zero real numbers, and let $N$ be a subgroup of $(\mathbb{R} \backslash\{0\}, \cdot)$. Then,

$$
|(\mathbb{R} \backslash\{0\}, \cdot) / N|<\aleph_{0} \Rightarrow\left(N=(\mathbb{R} \backslash\{0\}, \cdot) \vee N=\left(\mathbb{R}^{+}, \cdot\right)\right),
$$

i.e., $(\mathbb{R} \backslash\{0\}, \cdot) / N \cong(\{1\}, \cdot)$ or $(\mathbb{R} \backslash\{0\}, \cdot) / N \cong(\{-1,1\}, \cdot)$. Therefore, $(\mathbb{R} \backslash\{0\}, \cdot)$ is finitely stable having the finite shape of $(\{-1,1\}, \cdot)$. Clearly, the canonical $\aleph_{1}$-expansion of $(\mathbb{R} \backslash\{0\}, \cdot)$ reduces to the identity homomorphism.

9.2. The rudimentary commutative shape of groups. Let $G$ be a group, and let $\left[K_{G}\right]$ be its commutator subgroup. (If $G$ is Abelian, then $\left[K_{G}\right]=$ $\{e\} \unlhd G$.) Then the quotient group $G /\left[K_{G}\right]$ is commutative. Moreover, $\left[K_{G}\right]$ is the minimal such a normal subgroup, i.e., if $N \unlhd G$ such that $G / N$ is commutative, then $\left[K_{G}\right] \unlhd N$. Therefore, $G /\left[K_{G}\right]$ is the maximal quotient group of $G$ that is commutative. Let $p: G \rightarrow G /\left[K_{G}\right]$ be the quotient homomorphism. Given an $N \unlhd G$ such that $G / N$ commutative, let $p_{N}: G \rightarrow$ $G / N$ be the quotient homomorphism. Then there exists a unique relating (quotient) homomorphism $\tilde{p}$ of $G /\left[K_{G}\right]$ onto $G / N$ such that the diagram

$$
\begin{aligned}
& G /\left[K_{G}\right] \stackrel{p}{\leftarrow} \quad G \\
& \tilde{p} \downarrow \quad \swarrow p_{N} \\
& G / N
\end{aligned}
$$

in Grp commutes, i.e., $\tilde{p} p=p_{N}$. Notice that all the considered homomorphisms are epimorphic. Let us denote by eGrp $\subseteq$ Grp the subcategory of all groups and epimorphisms, and let $e A b \subseteq e G r p$ be the full subcategory determined by all Abelian groups.

LEMMA 9.5. The quotient homomorphism $p: G \rightarrow G /\left[K_{G}\right]$ is a rudimentary eAb-expansion of $G$. If $G$ is Abelian, then $p=1_{G}$.

Proof. Let $H$ be an Abelian group and let $f: G \rightarrow H$ be an epimorphism. Then $\operatorname{Ker}(f) \unlhd G$ such that $G / \operatorname{Ker}(f)$ is commutative because the 
homomorphism

$$
q: G / \operatorname{Ker}(f) \rightarrow H, \quad q p_{K e r(f)}=f,
$$

is an isomorphism. Let

$$
\tilde{p}: G /\left[K_{G}\right] \rightarrow G / \operatorname{Ker}(f), \quad \tilde{p} p=p_{K e r(f)} .
$$

be as above. Put

$$
g=q \tilde{p}: G /\left[K_{G}\right] \rightarrow H .
$$

Then $g$ is an epimorphism and $g p=f$. The conclusion follows.

REMARK 9.6. In order to obtain a less rudimentary shape, it would be interesting (in this approach) to replace abelian groups by the nilpotent ones. We postpone that work for an other occasion.

\section{The SHAPES OF RINGS}

Similarly to the cases of monoids and groups, an equivalence relation $\sim$ on a ring $(R,+, \cdot)$ is said to be a congruence if

$$
\left(\forall x, x^{\prime}, y, y^{\prime} \in R\right) \quad\left(x \sim x^{\prime} \wedge y \sim y^{\prime}\right) \Rightarrow\left(x+y \sim x^{\prime}+y^{\prime} \wedge x y \sim x^{\prime} y^{\prime}\right) .
$$

It is well known that a congruence $\sim$ is induced by an ideal $(A,+, \cdot) \unlhd(R,+, \cdot)$ and that $A=[0]$. We denote, as usually, the quotient ring $\mathrm{R} / \sim$ by $R / A$, and by $p: R \rightarrow R / A$ the quotient homomorphism. If $\Lambda$ is the set of all congruences $\lambda \equiv \sim$ on $R$, then we order $\Lambda$ by the refiniment. It corresponds to

$$
\lambda \leq \lambda^{\prime} \quad \Leftrightarrow \quad A_{\lambda^{\prime}} \leq A_{\lambda} \text { (the subring). }
$$

Since the intersection of any pair of ideals is an ideal, $\Lambda$ is directed as well. In the sequel, we shall abbreviate $R / A_{\lambda} \equiv R_{\lambda}$. If $f: R \rightarrow Q$ is a ring homomorphism that preserves given congruences $\sim \equiv \lambda$ and $\sim^{\prime} \equiv \mu$, i.e.,

$$
x \sim x^{\prime} \Rightarrow f(x) \sim^{\prime} f\left(x^{\prime}\right),
$$

then there exists a unique ring homomorphism $\tilde{f}: R_{\lambda} \rightarrow Q_{\mu}, \tilde{f}[x]=[f(x)]^{\prime}$, such that the following diagram

$$
\begin{array}{ccc}
R_{\lambda} & \stackrel{p_{\lambda}}{\leftarrow} & R \\
\tilde{f} \downarrow & & \downarrow f \\
Q_{\mu} & \stackrel{q_{\mu}}{\leftarrow} & Q
\end{array}
$$

(in the category Ring) commutes, i.e., $\tilde{f} p_{\lambda}=q_{\mu} f$. Therefore, if $\lambda \leq \lambda^{\prime}$, then $1_{R}: R \rightarrow R$ is $\left(\lambda, \lambda^{\prime}\right)$-preserving, and thus, there exists a unique ring epimorphism $p_{\lambda \lambda^{\prime}}: R_{\lambda^{\prime}} \rightarrow R_{\lambda}$ such that $p_{\lambda \lambda^{\prime}} p_{\lambda^{\prime}}=p_{\lambda}$. Further, for every ring homomorphism $f: R \rightarrow Q$, the equivalence relation $\sim_{f}$ induced by the fibers of $f, f^{-1}(y), y \in Q$, (i.e., by the kernel of $f$ ) is a congruence on $R$. Hence, there exists a $\lambda \in \Lambda$ such that $\sim_{f}=\lambda$, and there exists a unique ring homomorphism $f_{\lambda}: R_{\lambda} \rightarrow Q$ such that $f_{\lambda} p_{\lambda}=f$. If, in addition, $f$ is an 
(effective) epimorphism, then $f_{\lambda}$ is an isomorphism ("The ring isomorphism theorem"). In this way we have obtained an inverse system

$$
\boldsymbol{R}=\left(R_{\lambda}, p_{\lambda \lambda^{\prime}}, \Lambda\right)
$$

in Ring, and a morphism

$$
\boldsymbol{p}=\left(p_{\lambda}\right): R \rightarrow \boldsymbol{R}
$$

of pro-Ring that is a Ring-expansion of $R$. Since there exists $\lambda_{*}=\max \Lambda$ (corresponding to the trivial ideal $\{0\} \unlhd R$ ), and $R_{\lambda_{*}}=R$, we reduce $\Lambda$ so that the restriction remains an expansion. Now, as in all the cases before, we involve in our construction the cardinality of objects. Given an infinite cardinal $\kappa$, let $\Lambda_{\kappa^{-}}\left(\Lambda_{\kappa}\right)$ be the subset of $\Lambda$ consisting of all elements $\lambda$ such that $\left|R_{\lambda}\right|<\kappa\left(\left|R_{\lambda}\right| \leq \kappa\right)$. Then the restrictions of $\boldsymbol{p}$,

$$
\boldsymbol{p}_{\kappa^{-}}=\left(p_{\lambda}\right): R \rightarrow \boldsymbol{R}_{\kappa^{-}}=\left(R_{\lambda}, p_{\lambda \lambda^{\prime}}, \Lambda_{\kappa^{-}}\right)
$$

and

$$
\boldsymbol{p}_{\kappa}=\left(p_{\lambda}\right): R \rightarrow \boldsymbol{R}_{\kappa}=\left(R_{\lambda}, p_{\lambda \lambda^{\prime}}, \Lambda_{\kappa}\right)
$$

are the (canonical) Ring $_{\kappa^{-}}$-expansion and Ring $_{\kappa^{-}}$-expansion of $R$ respectively. This proves that $R i n g_{\kappa^{-}}$and $R i n g_{\kappa}$ are pro-reflective subcategories of Ring (of course, in the case of a finite ring $R$, one may choose, for every $\kappa^{-}$-and $\kappa$-expansion of $R$, the rudimentary identity expansion $\left\lfloor 1_{R}\right\rfloor: R \rightarrow R$ ).

Now one obtains, in the usual way, the shape categories $S h_{\kappa^{-}}(R i n g)$ and $S h_{\kappa}\left(\right.$ Ring), and the shape functors $S_{\kappa^{-}}: R i n g \rightarrow S h_{\kappa^{-}}(R i n g)$ and $S_{\kappa}: R i n g \rightarrow S h_{\kappa}($ Ring $)$, as well as the relating functor $S_{\kappa^{-} \kappa}: S h_{\kappa}(R i n g) \rightarrow$ $S h_{\kappa^{-}}\left(\right.$Ring) such that $S_{\kappa^{-}} S_{\kappa}=S_{\kappa^{-}}$.

EXAmple 10.1. Let $(\mathbb{Z},+, \cdot)$ be the ring of integers. Each subring of $\mathbb{Z}$ is a $k \mathbb{Z}, k \in\{0\} \cup \mathbb{N}$, that is also a principal ideal $(k) \unlhd \mathbb{Z}$. Further, given an $n \in\{0\} \cup \mathbb{N}$, each subring of $n \mathbb{Z}$ is a $k(n \mathbb{Z})=(k n) \mathbb{Z}, k \in\{0\} \cup \mathbb{N}$, that is also a principal ideal $(k n)$ of $n \mathbb{Z}$ and of $\mathbb{Z}$. Since a ring homomorphism of $\mathbb{Z}$ to itself is either $c_{0}$ or $1_{\mathbb{Z}}$, for every pair $n \neq n^{\prime}$, the rings $n \mathbb{Z}$ and $n^{\prime} \mathbb{Z}$ are not isomorphic. We will show that all of them, except $0 \mathbb{Z}$ - the trivial one, have the same finite shape, i.e., that

$$
\left(\forall n, n^{\prime} \in \mathbb{N}\right) \quad S h_{\underline{0}}(n \mathbb{Z},+, \cdot)=S h_{\underline{0}}\left(n^{\prime} \mathbb{Z},+, \cdot\right) .
$$

Recall that (the quotient rings)

$$
\mathbb{Z} /(k) \cong\left(\mathbb{Z}_{k},+_{k}, \cdot k\right),
$$

and

$$
n \mathbb{Z} /(k n) \cong\left(\mathbb{Z}_{k n},{ }_{k n}, \cdot{ }_{k n}\right) \cong \mathbb{Z} /(k n) .
$$

Therefore, the canonical Ring $_{0}$-expansion of $\mathbb{Z}$ is

$$
\boldsymbol{p}_{\underline{0}}=\left(p_{k}\right): \mathbb{Z} \rightarrow \boldsymbol{Z}_{\underline{0}}=\left(\mathbb{Z}_{k}, p_{k k^{\prime}},\left(\mathbb{N}, \leq^{*}\right)\right),
$$


where $\left(\mathbb{N}, \leq^{*}\right)$ is ordered (and directed) by

$$
k \leq^{*} k^{\prime} \Leftrightarrow \frac{k^{\prime}}{k} \in \mathbb{N}
$$

(see Example 1 (a)), while the canonical Ring $g_{0}$-expansion of $n \mathbb{Z}$ is

$$
\boldsymbol{p}_{\underline{0}}^{(n)}=\left(p_{k}\right): n \mathbb{Z} \rightarrow \boldsymbol{n} \boldsymbol{Z}_{\underline{0}}=\left(\mathbb{Z}_{k}, p_{k k^{\prime \prime}},\left(n \mathbb{N}, \leq^{*}\right)\right)
$$

(because $\left(n \mathbb{N}, \leq^{*}\right) \subseteq\left(\mathbb{N}, \leq^{*}\right)$ is directed as well). Finally, notice that each $\boldsymbol{p}_{0}^{(n)}$ is the restriction of $\boldsymbol{p}_{0}$ to $\left(n \mathbb{N}, \leq^{*}\right)$, and that $\left(n \mathbb{N}, \leq^{*}\right)$ is cofinal in $\left(\mathbb{N}, \leq^{*}\right)$ The conclusion follows (notice that $\boldsymbol{Z}_{\underline{0}}$ and $\boldsymbol{n} \boldsymbol{Z}_{\underline{0}}$ are not isomorphic to any inverse sequence - see Example 15).

ExAmple 10.2. Since the ring $(\mathbb{R},+, \cdot)$ of reals is a field (there is no non-trivial ideal), its $\underline{0}$-shape and $\aleph_{0}$-shape are trivial, while the canonical $\aleph_{1}$-expansion of $(\mathbb{R},+, \cdot)$ obviously reduces to the identity ring homomorphism

EXAmPLE 10.3. Similarly to Example 18 , the $\underline{0}$-shape of the ring $(\mathbb{Q},+, \cdot)$ of rationals is trivial, while the canonical $\aleph_{0}$-expansion of $(\mathbb{Q},+, \cdot)$ reduces to the identity ring homomorphism.

REMARK 10.4. In order to obtain the (quotient) shape theories for a subcategory of Ring with respect to a special full subcategory, one has to assure that the special properties are preserved by the quotients and (finite) intersections. An interesting special category could be the full subcategory of all polynomial rings over a class of fields, while for its special subcategory one may choose, for instance, that of commutative rings, integral (or some more special) domains or (skew) fields (see [6], Chapter XI).

\section{The Shapes OF MOdUles}

Recall (see [12]) that a (left) $R$-module is every ordered pair $(M, \varphi)$ consisting of an Abelian group $M$ and a function $\varphi: R \times M \rightarrow M, \varphi(r, x) \equiv r x$, where $R$ is a ring with unity 1 , such that

$$
r\left(x+x^{\prime}\right)=r x+r x^{\prime},\left(r+r^{\prime}\right) x=r x+r^{\prime} x \text { and } r\left(r^{\prime} x\right)=\left(r r^{\prime}\right) x, 1 x=x
$$

always hold. In the sequel, we shall not stress $\varphi$ unless necessary. Clearly, every ring $R$ with unity is itself an $R$-module with $\varphi$ the ring multiplication. Let $R$-Mod denote the category of all (left) $R$-modules and all (left) $R$-module homomorphisms $f: M \rightarrow N$,

$$
f(x+y)=f(x)+f(y) \text { and } \quad f(r x)=r f(x) .
$$

A subset $S$ of an $R$-module $M$ is a (left $R$-) submodule, denoted by $S \unlhd M$, if it is an $R$-module with respect to the same operation. This means that $S$ is a subgroup of (the Abelian group) $M$ and that, for every $r \in R, r S \subseteq S$ (likewise to a left ideal of a ring). It follows that a submodule of a submodule of $M$ is a submodule of $M$. If $S$ and $T$ are submodules of $M$, then so is 
$S \cap T$. For every submodule $S$ of $M$, the inclusion $i: S \hookrightarrow M$ is an $R$-module monomorphism. Further, the quotient $R$-module

$$
M / S=\{[x]=x+S \mid x \in M\}, \quad[x]+[y]=[x y], \quad r[x]=[r x],
$$

and the quotient $R$-module (effective) epimorphisms (the canonical projection)

$$
p: M \rightarrow M / S, \quad p(x)=[x],
$$

are well defined. Let $M$ be an $R$-module.

An equivalence relation $\sim$ on a set $M$ is a congruence on the $R$-module $M$ if the equivalence class [0] $\subseteq M$ is a submodul of $M$. If $\Lambda$ is the set of all congruences $\lambda \equiv \sim$ on $M$, we order it by the refinement, i.e., $\lambda \leq \lambda^{\prime}$ if and only if $\lambda^{\prime}$ refines $\lambda$. Equivalently,

$$
\lambda \leq \lambda^{\prime} \Leftrightarrow S_{\lambda^{\prime}} \unlhd S_{\lambda} \text { (the submodule). }
$$

Then $(\Lambda, \leq)$ becomes a partially ordered set, that is directed as well. Let us denote $M / S_{\lambda}=M_{\lambda}$ and $p=p_{\lambda}: M \rightarrow M_{\lambda}$. If $f: M \rightarrow N$ is an $R$-module homomorphism that is $\left(\lambda \equiv \sim, \mu \equiv \sim^{\prime}\right)$-preserving, i.e.,

$$
x \sim x^{\prime} \Rightarrow f(x) \sim^{\prime} f\left(x^{\prime}\right)
$$

(equivalently, $\left.f\left(\left[0_{M}\right]\right) \subseteq\left[0_{N}\right]^{\prime}\right)$, then there exists a unique $R$-module homomorphism $\tilde{f}: M_{\lambda} \rightarrow N_{\mu}, \tilde{f}[x]=[f(x)]^{\prime}$, such that the following diagram

$$
\begin{array}{lll}
M_{\lambda} & \stackrel{p_{\lambda}}{\leftarrow} & M \\
\tilde{f} \downarrow & & \downarrow f \\
N_{\mu} & \stackrel{q_{\mu}}{\leftarrow} & N
\end{array}
$$

(in the category $R$-Mod) commutes, i.e., $\tilde{f} p=q f$. Therefore, if $\lambda \leq \lambda^{\prime}$, then $1_{M}: M \rightarrow M$ is $\left(\lambda, \lambda^{\prime}\right)$-preserving, and thus, there exists a unique $R$-module epimorphism $p_{\lambda \lambda^{\prime}}: M_{\lambda^{\prime}} \rightarrow M_{\lambda}$ such that $p_{\lambda \lambda^{\prime}} p_{\lambda^{\prime}}=p_{\lambda}$. Further, for every $R$-module homomorphism $f: M \rightarrow N$, the equivalence relation $\sim_{f}$ induced by the fibers of $f, f^{-1}(y), y \in Q$, (i.e., by the kernel $\left.\operatorname{Ker}(f)\right)$ is a congruence on $M$. Hence, there exists a $\lambda \in \Lambda$ such that $\sim_{f}=\lambda$, and there exists a unique $R$-module homomorphism $f_{\lambda}: M_{\lambda} \rightarrow N$ such that $f_{\lambda} p_{\lambda}=f$. If, in addition, $f$ is an epimorphism, then $f_{\lambda}$ is an isomorphism ("The $R$-module isomorphism theorem"). Consequently, there exists a certain inverse system

$$
\boldsymbol{M}=\left(M_{\lambda}, p_{\lambda \lambda^{\prime}}, \Lambda\right)
$$

in $R-M o d$, and a morphism

$$
\boldsymbol{p}=\left(p_{\lambda}\right): M \rightarrow \boldsymbol{M}
$$

of pro- $(R-M o d)$ that is a $R$-Mod-expansion of $M$. Since there exists $\lambda_{*}=$ $\max \Lambda$ (corresponding to the trivial submodule $\{0\} \unlhd M\}$ ), and $M_{\lambda_{*}}=M$, $\boldsymbol{p}$ is equivalent to the rudimentary identity $\left\lfloor 1_{M}\right\rfloor$. In order to obtain a nonrudimentary expansion, we have to reduce $\Lambda$ so that the restriction remains an expansion. Now, as in all the cases before, given an infinite cardinal $\kappa$, 
let $\Lambda_{\kappa^{-}}\left(\Lambda_{\kappa}\right)$ be the subset of $\Lambda$ consisting of all $\lambda \in \Lambda$ such that $\left|M_{\lambda}\right|<\kappa$ $\left(\left|M_{\lambda}\right| \leq \kappa\right)$. Then the restrictions of $\boldsymbol{p}$,

$$
\boldsymbol{p}_{\kappa^{-}}=\left(p_{\lambda}\right): M \rightarrow \boldsymbol{M}_{\kappa^{-}}=\left(M_{\lambda}, p_{\lambda \lambda^{\prime}}, \Lambda_{\kappa^{-}}\right)
$$

and

$$
\boldsymbol{p}_{\kappa}=\left(p_{\lambda}\right): M \rightarrow M_{\kappa}=\left(M_{\lambda}, p_{\lambda \lambda^{\prime}}, \Lambda_{\kappa}\right)
$$

are the (canonical) $R$-Mod $\kappa_{\kappa^{-}}$-expansion and $R$ - ood $_{\kappa^{-}}$-expansion of $M$ respectively. This shows that $R-\operatorname{Mod}_{\kappa^{-}}$and $R-\operatorname{Mod}_{\kappa}$ are pro-reflective subcategories of $R$-Mod. Similarly to the previous cases, if $\kappa>|M|$, then $\boldsymbol{p}_{\kappa^{-}}$is isomorphic to the rudimentary expansion $\left\lfloor 1_{M}\right\rfloor$, while $\kappa \geq|M|$ implies that $\boldsymbol{p}_{\kappa}$ is isomorphic to the rudimentary expansion of $M$.

Now one obtains, in the usual way, the shape categories $S h_{\kappa^{-}}(R-M o d)$ and $S h_{\kappa}(R-M o d)$, and the shape functors $S_{\kappa^{-}}: R$-Mod $\rightarrow S h_{\kappa^{-}}(R-M o d)$ and $S_{\kappa}: R-M o d \rightarrow S h_{\kappa}(R-M o d)$, as well as the relating functor $S_{\kappa^{-} \kappa}: S h_{\kappa}(R-$ $M o d) \rightarrow S h_{\kappa^{-}}(R-M o d)$ such that $S_{\kappa^{-}} S_{\kappa}=S_{\kappa^{-}}$.

Since each ring $R$ with unity is an $R$-module, the shape classifications in $R-M o d$ are, according to Section 10, strictly coarser than the classification by the $R$-module isomorphisms. The same conclusion holds, by Section 9 , for the category $\mathbb{Z}$-Mod because every $\mathbb{Z}$-module is just the Abelian group. We provide hereby several canonical expansions of some "not very special" $R$-modules $M$, where $M$ is not isomorphic to $\mathbb{Z}$ or $\mathbb{R}$.

ExAMPLE 11.1. (a) Let $((\mathbb{Z},+), \varphi)$ be the $\operatorname{Hom}(\mathbb{Z})$-module (the ring of all endomorphisms on $(\mathbb{Z},+)$ with respect to the addition and composition of functions), where

$$
\varphi: \operatorname{Hom}(\mathbb{Z}) \times \mathbb{Z} \rightarrow \mathbb{Z}, \quad \varphi(h, m) \equiv h m=h(m) .
$$

One verifies the needed conditions straightforwardly. Notice that

$$
\varphi(h, m \cdot n)=m \cdot \varphi(h, n) \quad(\text { i.e., } h(m n)=m h(n))
$$

holds as well. Therefore, $M \subseteq((\mathbb{Z},+), \varphi)$ is an $\operatorname{Hom}(\mathbb{Z})$-submodule if and only if $M$ is an ideal of the ring $(\mathbb{Z},+, \cdot)$. Consequently, every submodule is a principal ideal $n \mathbb{Z}, n \in\{0\} \cup \mathbb{N}$. Obviously, the $\operatorname{Hom}(\mathbb{Z})$-modules $((\mathbb{Z},+), \varphi)$, $((n \mathbb{Z},+), \varphi \mid(-\times n \mathbb{Z}))$ and $\left(\left(n^{\prime} \mathbb{Z},+\right), \varphi \mid\left(-\times n^{\prime} \mathbb{Z}\right)\right), n \neq n^{\prime}$, are not isomorphic. However, by following the pattern of Example 17, if $n \neq 0 \neq n^{\prime}$, the corresponding canonical $\operatorname{Hom}(\mathbb{Z})$-Mod $d_{\underline{0}}$-expansions $\boldsymbol{p}_{\underline{0}}, \boldsymbol{p}_{\underline{0}}^{(n)}$ and $\boldsymbol{p}_{\underline{0}}^{\left(n^{\prime}\right)}$ show that they have the same (finite) $\operatorname{Hom}(\mathbb{Z})-\operatorname{Mod}_{\underline{0}}$-shape.

ExAmPLE 11.2. Let $((\mathbb{R},+), \varphi)$ be the $\mathbb{Q}$-module (the ring of rationals), where

$$
\varphi: \mathbb{Q} \times \mathbb{R} \rightarrow \mathbb{R}, \quad \varphi(q, x)=q x \text { (the multiplication) }
$$

Since $\mathbb{Q}$ is a field, the $\mathbb{Q}$-module $((\mathbb{R},+), \varphi)$ is, actually, the (uncountably infinite-dimensional) vectorial space $\mathbb{R}_{\mathbb{Q}}$. Hence, every its submodule is a vectorial subspace of $\mathbb{R}_{\mathbb{Q}}$. Each 1-dimensional subspace is generated by a 
single vector $x \in \mathbb{R}$. For instance, the field as a subspace $\mathbb{Q} \unlhd \mathbb{R}_{\mathbb{Q}}$ is generated by any $x \in \mathbb{Q}$. A 2 -dimensional subspace is generated by any two vectors $x, y \in \mathbb{R}$ that are rationally-linearly independent, i.e., $y \neq q x$ for every $q \in$ $\mathbb{Q}$. A "smalll" quotient modul (space) asks for a certain "large" submodule (subspace). However, every quotient $\mathbb{Q}$-module is a vectorial space over $\mathbb{Q}$. The consequence is that, except $\{0\}=\mathbb{R} / \mathbb{R}$ - the trivial one, there is no quotient $\mathbb{Q}$-modul of $\mathbb{R}_{\mathbb{Q}}$ having a finite cardinality. Hence, the canonical $\mathbb{Q}$ - Mod $_{\underline{0}}$-expansion of $\mathbb{R}_{\mathbb{Q}}$ is rudimentary trivial, i.e., it is

$$
\boldsymbol{p}_{\underline{0}}=\left(c_{0}\right): \mathbb{R}_{\mathbb{Q}} \rightarrow\lfloor\{0\}\rfloor \text {. }
$$

Thus, $\mathbb{R}_{\mathbb{Q}}$ has trivial (finite) $\mathbb{Q}$ - $M$ od $\underline{0}$-shape. Further, since $\mathbb{Q}$ is countable, each countable quotient $\mathbb{Q}$-module $R_{\lambda}$ of $\mathbb{R}_{\mathbb{Q}}$ is obtained via a submodule $L_{\lambda}$ that is a vectorial subspaces generated by a certain subset $\lambda \subseteq \mathbb{R}$ having countable complement. Thus, the canonical $\mathbb{Q}-\operatorname{Mod}_{\aleph_{0}}$-expansion of $\mathbb{R}_{\mathbb{Q}}$ is

$$
\boldsymbol{p}_{\aleph_{0}}=\left(p_{\lambda}\right): \mathbb{R}_{\mathbb{Q}} \rightarrow\left(R_{\lambda}, p_{\lambda \lambda^{\prime}}, \Lambda_{\aleph_{0}}\right),
$$

where $|\Lambda|=2^{c}$. Finally, for every cardinal $\kappa>c\left(\kappa>2^{\aleph_{0}}-\right.$ by $\left.\mathrm{CH}\right)$, the canonical $\mathbb{Q}$ - $\operatorname{Mod}_{\kappa^{-}}$-expansion $\boldsymbol{p}_{\kappa^{-}}$reduces to the rudimentary identity

$$
\boldsymbol{p}_{\kappa^{-}}=\left(1_{\mathbb{R}_{\mathbb{Q}}}\right): \mathbb{R}_{\mathbb{Q}} \rightarrow\left\lfloor\mathbb{R}_{\mathbb{Q}}\right\rfloor,
$$

as well as $\boldsymbol{p}_{\kappa}$ for $\kappa \geq c\left(\kappa \geq 2^{\aleph_{0}}-\right.$ by $\left.\mathrm{CH}\right)$.

REMARK 11.3. Everything we have established concerning left $R$-modules $(R-M o d)$ holds, mutatis mutandis, for right $R$-modules $(M o d-R)$ as well.

\section{The (Algebraic) SHAPES of VeCtorial SPACES}

The last example of the previous section motivates the following idea concerning the quotient shapes of vectorial spaces (that is compatible with our general principle): We propose that every finite-dimensional vectorial space $X$ is "nice", and if $X$ is infinite- $\kappa$-dimensional, then every vectorial space having dimension less than $\kappa$ is "nice comparing to" $X$. As usually, $\operatorname{dim} X=|B|$, where $B$ is an algebraic (Hamel) basis of $X$. Given a field $F$, let $V e c t_{F}$ denote the category of all vectorial spaces over $F$ and all their linear functions. It is more convenient, and in some special considerations it is equivalent, to deal with a basis of a space than with the family of all its subspaces. However, for our purpose, the linear hulls of all subsets of a given basis (only) are not sufficient to obtain an expansion of $X$. So we must consider all the subspaces of a given infinite-dimensional space.

Let $X \in O b\left(\right.$ Vect $\left._{F}\right)$ and let $\left\{Z_{\lambda} \mid \lambda \in \Lambda\right\}$ be the set of all its subspaces $Z_{\lambda} \unlhd X$. We define

$$
\lambda \leq \lambda^{\prime} \Leftrightarrow Z_{\lambda^{\prime}} \unlhd Z_{\lambda} .
$$

Then $(\Lambda, \leq)$ is a poset, that is directed because of

$$
Z_{\lambda^{\prime \prime}} \unlhd Z_{\lambda} \cap Z_{\lambda^{\prime}} \Leftrightarrow \lambda^{\prime \prime} \geq \lambda, \lambda^{\prime} .
$$


For every $\lambda \in \Lambda$, put

$$
X_{\lambda} \equiv X / Z_{\lambda}=\left\{[x]_{\lambda}=x+Z_{\lambda} \mid x \in X\right\}
$$

to be the quotient vectorial space, and let

$$
p_{\lambda}: X \rightarrow X_{\lambda}, \quad p_{\lambda}(x)=[x]_{\lambda},
$$

be the canonical projection, which is a linear function. Notice that $\operatorname{dim} X=$ $\operatorname{dim} X_{\lambda}+\operatorname{dim} Z_{\lambda}$. Given a pair $\lambda \leq \lambda^{\prime}$ (i.e., $Z_{\lambda^{\prime}} \unlhd Z_{\lambda}$ ), let

$$
p_{\lambda \lambda^{\prime}}: X_{\lambda^{\prime}} \rightarrow X_{\lambda}, \quad p\left([x]_{\lambda^{\prime}}\right)=[x]_{\lambda},
$$

be the relating function, which is linear as well. Then, $p_{\lambda \lambda^{\prime}} p_{\lambda^{\prime}}=p_{\lambda}$ and $p_{\lambda \lambda^{\prime}} p_{\lambda^{\prime} \lambda^{\prime \prime}}=p_{\lambda \lambda^{\prime \prime}}$, whenever $\lambda \leq \lambda \leq \lambda^{\prime \prime}$. In this way a certain inverse system $\boldsymbol{X}=\left(X_{\lambda}, p_{\lambda \lambda^{\prime}}, \Lambda\right)$ in Vect $_{F}$ is associated with $X$, as well as, a morphism

$$
\boldsymbol{p}=\left(p_{\lambda}\right): X \rightarrow \boldsymbol{X}
$$

of pro-Vect $F_{F}$ such that all $p_{\lambda}$ and all $p_{\lambda \lambda^{\prime}}$ are (effective) epimorphisms. Since there exists $\lambda_{*}=\max (\Lambda, \leq)=\varnothing$ (corresponding to the trivial subspace $Z_{\lambda_{*}}=\{\theta\}$ ), and $X_{\lambda_{*}} \cong X$, the system $\boldsymbol{X}$ is isomorphic (in pro-Vect pro ) to the rudimentary system $\lfloor X\rfloor$ of $X$. However, given an infinite cardinal $\kappa$, one can consider the restriction to the subset $\Lambda_{\kappa^{-}} \subseteq \Lambda\left(\Lambda_{\kappa} \subseteq \Lambda\right)$ of all $\lambda \in \Lambda$ such that $\operatorname{dim} X_{\lambda}<\kappa\left(\operatorname{dim} X_{\lambda} \leq \kappa\right)$ : Then one straightforwardly proves that $\left(\Lambda_{\kappa^{-}}, \leq\right)\left(\left(\Lambda_{\kappa}, \leq\right)\right)$ is a directed set. Moreover, there exists a morphism

$$
\begin{gathered}
\boldsymbol{p}_{\kappa^{-}}=\left(p_{\lambda}\right): X \rightarrow \boldsymbol{X}_{\kappa^{-}}=\left(X_{\lambda}, p_{\lambda \lambda^{\prime}}, \Lambda_{\kappa^{-}}\right) \\
\left(\boldsymbol{p}_{\kappa}=\left(p_{\lambda}\right): X \rightarrow \boldsymbol{X}_{\kappa}=\left(X_{\lambda}, p_{\lambda \lambda^{\prime}}, \Lambda_{\kappa}\right)\right),
\end{gathered}
$$

of pro- $\left(\text { Vect }_{F}\right)_{\kappa^{-}}\left(\right.$pro- $\left.\left(\text {Vect }_{F}\right)_{\kappa}\right)$.

Clearly, if $\kappa>\operatorname{dim} X$, then $\boldsymbol{p}_{\kappa^{-}}$is isomorphic to the rudimentary expansion $\left\lfloor 1_{X}\right\rfloor$ of $X$, while $\kappa \geq \operatorname{dim} X$ implies that $\boldsymbol{p}_{\kappa}$ is isomorphic to the rudimentary expansion $\left\lfloor 1_{X}\right\rfloor$ of $X$. We are to prove that $\boldsymbol{p}_{\kappa^{-}}: X \rightarrow \boldsymbol{X}_{\kappa^{-}}$ $\left(\boldsymbol{p}_{\kappa}: X \rightarrow \boldsymbol{X}_{\kappa}\right)$ is a $\left(\text { Vect }{ }_{F}\right)_{\kappa^{-}}$-expansion $\left(\left(V e c t_{F}\right)_{\kappa^{-}}\right.$expansion $)$of $X$.

THEOREM 12.1. For every infinite cardinal $\kappa,\left(\text { Vect }_{F}\right)_{\kappa^{-}}$and $\left(\text {Vect }_{F}\right)_{\kappa}$ are pro-reflective subcategories of $V$ ect ${ }_{F}$.

Proof. It suffices to verify condition (E1) for $\boldsymbol{p}_{\kappa^{-}}$and $\boldsymbol{p}_{\kappa}$,

$$
\begin{aligned}
& \boldsymbol{p}_{\kappa^{-}}=\left(p_{\lambda}\right): X \rightarrow \boldsymbol{X}_{\kappa^{-}}=\left(X_{\lambda}, p_{\lambda \lambda^{\prime}}, \Lambda_{\kappa^{-}}\right), \\
& \boldsymbol{p}_{\kappa}=\left(p_{\lambda}\right): X \rightarrow \boldsymbol{X}_{\kappa}=\left(X_{\lambda}, p_{\lambda \lambda^{\prime}}, \Lambda_{\kappa}\right),
\end{aligned}
$$

constructed previously. If $\kappa>\operatorname{dim} X$, then (E1) for $\boldsymbol{p}_{\kappa^{-}}$holds trivially. Let $\kappa \leq \operatorname{dim} X$. Let $Y$ be any vectorial space (over the same field) such that $\operatorname{dim} Y<\kappa$, and let $f: X \rightarrow Y$ be a linear function. Then the kernel $N(f) \unlhd X$ and there exists a unique linear function $\tilde{f}: X / N(f) \rightarrow Y$ such that $\tilde{f} p=f$, where $p: X \rightarrow X / N(f)$ is the quotient (linear) epimorphism. Clearly, there exists a $\lambda \in \Lambda$ such that $N(f)=Z_{\lambda}$, and thus, $X / N(f)=X_{\lambda}$ and $p=p_{\lambda}: X \rightarrow X_{\lambda}$. Since the image $R(f) \unlhd Y$, it follows that $\operatorname{dim} R(f)<\kappa$. 
Finally, $X \cong N(f) \oplus R(f)$ implies that $\operatorname{dim} X_{\lambda}=\operatorname{dim} R(f)<\kappa$, which shows that $\lambda \in \Lambda_{\kappa^{-}}$. In the $\kappa$-case, if $\kappa \geq \operatorname{dim} X$, then condition (E1) for $\boldsymbol{p}_{\kappa}$ holds trivially; if $\kappa<\operatorname{dim} X$, one can verify (E1) for $\boldsymbol{p}_{\kappa}$ in the same way as for $\boldsymbol{p}_{\kappa^{-}}$.

We conclude this paper with the next final remark.

REMARK 12.2. (a) It seems that all the algebraic quotient shape classifications and the isomorphism classification of vectorial spaces coincide. Namely, they reduce to the cardinalities of bases (without any structure), and this leads to the category Set (Section 4). Thus, in order to obtain a coarser quotient classification, one should abandon the pure algebraic approach and consider $V e c t_{F}$ endowed with an extra structure. Instead of the algebraic ( $\mathrm{Hamel}$ ) bases one might consider topological (Schauder) bases The class of all normed vectorial (primarily, infinite dimensional Banach) spaces admitting Shauder bases might be a convenient one for the (finite) shape classification.

(b) In the special case of the category $A A l_{F} \subseteq V e c t_{F}$ of all associative algebras over $F$ (and their operators), for every infinite-dimensional algebra, $X$, a congruence class $[0] \unlhd X$ has to be an ideal of $(X,+, \cdot)$ as well.

\section{ACKNOWLEDGEMENT.}

The author would like to thank the referee for his/her constructive criticism, valuable comments, corrections and helpful suggestions concerning the whole concept as well as many specific details.

\section{REFERENCES}

[1] K. Borsuk, Concerning homotopy properties of compacta, Fund. Math. 62 (1968), $223-254$.

[2] K. Borsuk, Theory of shape, Matematisk Inst. Aarhus Univ., Aarhus, 1971.

[3] K. Borsuk, Theory of shape, Polish Scientific Publishers, Warszaw, 1975.

[4] J.-M. Cordier and T. Porter, Shape theory. Categorical methods of approximation, Ellis Horwood Ltd., Chichester, 1989. (Dover edition, 2008.)

[5] J. Dugundji, Topology, Allyn and Bacon, Boston, 1966.

[6] J. R. Durbin, Modern algebra. An introduction, John Wiley \& Sons, Inc., New York, 1992.

[7] J. Dydak and J. Segal, Shape theory. An introduction, Springer-Verlag, Berlin, 1978.

[8] D. A. Edwards and H. M. Hastings, Čech and Steenrod homotopy theories with applications to geometric topology, Springer-Verlag, Berlin, 1976.

[9] S. Eilenberg and N. Steenrod, Foundations of algebraic topology, Princeton University Press, Princeton, 1952.

[10] H. Herlich and G. E. Strecker, Category theory. An introduction, Allyn and Bacon Inc., Boston, 1973.

[11] N. Koceić Bilan and N. Uglešić, The coarse shape, Glas. Mat. Ser. III. 42(62) (2007), $145-187$.

[12] S. Mac Lane, Homology, Springer-Verlag, Berlin, 1995.

[13] S. Mardešić and J. Segal, Shape theory. The inverse system approach, Noth-Holland Publishing co., Amsterdam-New York, 1982. 
[14] I. Moerdijk, Prodiscrete groups and Galois toposes, Nederl. Akad. Wetensch. Indag. Math. 51 (1989), 219-234.

[15] W. Tholen, Pro-categories and multiadjoint functors, Canad. J. Math. 36 (1984), $144-155$.

[16] N. Uglešić, On ultrametrics and equivalence relations-duality, Int. Math. Forum 5 (2010), 1037-1048.

[17] N. Uglešić and V. Matijević, On expansions and pro-pro-categories, Glas. Mat. Ser. III. 45(65) (2010), 173-217.

[18] N. Uglešić and B. Červar, The concept of a weak shape type, Int. J. Pure Appl. Math. 39 (2007), 363-428.

N. Uglešić

University of Zadar

Pavlinovićeva 1, 23000 Zadar

Croatia

E-mail: nuglesic@unizd.hr

Received: 1.6.2015.

Revised: 23.9.2015. \& 25.1.2016. 